\title{
CLASSIFICATION OF DIRECT LIMITS OF GENERALIZED TOEPLITZ ALGEBRAS
}

\author{
Huaxin Lin And Hongbing Su
}

\begin{abstract}
A classification is given to the class of $C^{*}$-algebras of real rank zero which are direct limits of so called generalized Toeplitz algebras. This class includes all so called $A \mathbb{T}$-algebras of real rank zero as well as many $C^{*}$-algebras which are not stably finite.
\end{abstract}

\section{Introduction.}

A certain class of $C^{*}$-algebras is classified. The class consists $C^{*}$-algebras of real rank zero that can be expressed as direct limits of finite direct sums of matrix algebras over $\mathcal{T}$-algebras, where $\mathcal{T}$-algebras are unital essential extensions of $C\left(S^{1}\right)$ by compact operators $\mathcal{K}$ :

$$
0 \rightarrow \mathcal{K} \rightarrow E \rightarrow C\left(S^{1}\right) \rightarrow 0 .
$$

Let $A$ be an algebra in the class. The invariant consists of the abelian semigroup $V(A)$, the Murry-von Neumann equivalence classes of projections in matrices of $A$, an abelian semigroup $k(A)_{+}$, some equivalence classes of homotopy classes of hyponormal partial isometries in matrices of $A$ and a homomorphism $d$ from $k(A)_{+}$into $V(A)$. The main result of this paper states that the above invariant, together with the class of the identity, is complete for the class of $C^{*}$-algebras that we consider (cf. §5). For the subclass of the above class which consists of direct limits of finite direct sums of matrix algebras over only non-trivial extensions of the above type, the invariants are even simplier-just $V(A)$. We also show that the algebras in the class exhaust all possible invariants (cf. 3.7).

Our paper can be viewed as part of the program of classifying "amenable" $C^{*}$-algebras initiated by George A. Elliott. The classical model for the program is the classification of $A F$-algebras by their dimension groups [Ell1]. Elliott proved in [E112] that the class of real rank zero $A \mathbb{T}$-algebras, direct limits of finite direct sums of matrix algebras over $C\left(S^{1}\right)$ of real rank zero, could be classified by the graded group $K_{0} \oplus K_{1}$ with its natural order. Since then a number of classification results appeared ([BEEK], [D1], [D2], [DL1], [DL2], [E113], [E114], [El15], [EE], [EG1], [EG2], [EGL], [EGLP], 
[ER], [G1], [G2], [G3], [K], [Li], [Ln2], [Ln3], [Ln5], [LP1], [LP2], [LP3], [Lr], [Ph1], [Ph2], [Rr1], [Rr2], [Rr3], [Rr4], [Rr5], [Su1], [Su2], [Tm], etc.). Many classes of $C^{*}$-algebras are classified. These include $C^{*}$-algebras of real rank zero which are direct limits of homogeneous algebras of slow dimension growth and a large class (presumably all) separable nuclear purely infinite simple $C^{*}$-algebras. The situation is changing even when we are typing this paper. $C^{*}$-algebras that we consider in this paper are usually nonsimple and their stable rank are usually greater than one. The class does contain all $A \mathbb{T}$-algebras of real rank zero. It contains many other $C^{*}$-algebras which are not of so called approximately homogeneous $C^{*}$-algebras. One of the important features which makes our class essentially different from approximately homogeneous algebras is that the torsion in $K_{0}$ does not arise from the torsion parts of certain metric spaces but from nontrivial extensions of $C\left(S^{1}\right)$ by $\mathcal{K}$.

Inductive limits of finite direct sums of matrix algebras over the classical Toeplitz algebra $\mathcal{T}_{1}$ (i.e. the extension with index -1) was first studied in [EES]. A stable isomorphism theorem was obtained for the special case that each summand is a single matrix algebra over $\mathcal{T}_{1}$ and the algebras are of real rank zero. In [EES], the algebras in question were shown to be absorbing extensions of real rank zero $A \mathbb{T}$-algebras by stable AF algebras, which led to the stable classification.

The class that we consider here allows building blocks to be matrix algebras over any unital essential extensions of $C\left(S^{1}\right)$ by $\mathcal{K}$. This gives us torsion in $K$-theory as well as non-trivial $K_{1}$-theory. We also obtained a classification up to isomorphism instead of stable isomorphism.

One of the technical problems that we have to deal with is to lift two "close" homomorphisms from $M_{k}\left(C\left(S^{1}\right)\right)$ into $M_{m}\left(C\left(S^{1}\right)\right)$ to two "close" homomorphisms from $M_{k}\left(\mathcal{T}_{i}\right)$ into $M_{m}\left(\mathcal{T}_{j}\right)$. Here, $\mathcal{T}_{i}$ and $\mathcal{T}_{j}$ are the extensions of $C\left(S^{1}\right)$ by $\mathcal{K}$ with indexes $-i$ and $-j$, respectively (cf. 2.1). There are several problems here. First, not every homomorphism from $M_{k}\left(C\left(S^{1}\right)\right)$ into $M_{m}\left(C\left(S^{1}\right)\right)$ comes from an injective homomorphism from $M_{k}\left(\mathcal{T}_{i}\right)$. Second, two quite different homomorphisms from $M_{k}\left(\mathcal{T}_{i}\right)$ into $M_{m}\left(\mathcal{T}_{j}\right)$ which map $M_{k}\left(\mathcal{T}_{i}\right)$ to $M_{m}(\mathcal{K})$ induce two zero homomorphisms from $M_{k}\left(C\left(S^{1}\right)\right)$ to $M_{m}\left(C\left(S^{1}\right)\right)$. However, there is nothing to lift. Third, assuming the life is perfect, $k=m, i=j$ and two homomorphisms are two automorphisms on $M_{k}\left(\mathcal{T}_{i}\right)$ which induce the identity map on $C\left(S^{1}\right)$. How much can we say about these two automorphisms?

It turns out that the first two problems could be avoided using some existing tricks. For the third problem, we establish a so called uniqueness theorem (Section 4). It uses the BDF-theory as well as Voiculescu's generalized Weyl-von Neumann theorem. A special case of one of the lemmas says 
that an automorphism $\alpha$ of the Toeplitz algebra which has the property that $\pi \circ \alpha=\pi$, where $\pi$ is the quotient map from the Toeplitz algebra onto the circle algebra $C\left(S^{1}\right)$, is approximately inner, a result has been just obtained by Rørdam independently with a completely different proof.

Since we allow the building blocks to include trivial extension $\mathcal{T}_{0}$, the invariants become more complicated. For example, $V\left(\mathcal{T}_{0}\right)$ is not finitely generated. This causes lots of problems when we try to employ a standard intertwining argument. For this, we have to do some surgical work.

We have some description (3.4) of the invariants that we use here. It would be nicer to have a characterization of our invariants. A version of Effros-Handelmen-Shen theorem which characterizes the dimension groups or an Elliott's theorem ([E116]) is certainly desirable. However, at present, it is beyond our reach to characterize these semigroups and we feel that it should be treated separately.

A $C^{*}$-algebra in the class often becomes an essential extension of an $A \mathbb{T}$ algebra by an AF-algebra. But this paper is not about classification of extensions. Classification of extensions requires to know the ideal and the quotient. Our invariant is defined for general $C^{*}$-algebras which does not require to know the ideal nor the quotient. In fact, a $C^{*}$-algebra in the class may be written into different extensions. In other words, a result of classification of extensions of a given $A \mathbb{T}$-algebra by a given AF-algebra, while is important, would not classify our class.

The paper is organized as follows. In Section 2, we define and discuss the invariants of $A \mathcal{T}$-algebras. It is rather tedious section. In Section 3, we establish an existence theorem. We further show that given a possible invariant $V_{*}$ there is a direct limit $A$ of finite direct sums of matrix algebras over $A \mathcal{T}$-algebras with real rank zero such that $V_{*}(A)=V_{*}$. We also show that every $A \mathbb{T}$-algebra of real rank zero can be expressed as a direct limit of finite direct sums of matrix algebras over $A \mathcal{T}$-algebras. In Section 4 , we give the uniqueness theorem. In Section 5, we combine the results in the previous sections and give the main result.

Acknowledgement. The first author acknowledges the support from the Fields Institute during a summer visit in 1994 when a draft of this paper was initially written. He is also supported by a NSF grant. The second author is supported by a NSERC postdoctoral fellowship of Canada. He would like to thank G.A. Elliott, D.D. Evans, G. Gong and D. Voiculescu for helpful discussions. 


\section{2. $\mathcal{T}$-algebras and their invariants.}

2.1. Let $C\left(S^{1}\right)$ be the continuous functions on the unit circle and let $\mathcal{K}$ be the compact operators on an infinite dimensional separable Hilbert space. The generalized Toeplitz algebras $\mathcal{T}_{k}$ is an essential unital extension of $C\left(S^{1}\right)$ by $\mathcal{K}$ with index $-k \in \mathbf{Z}$ :

$$
0 \rightarrow \mathcal{K} \rightarrow \mathcal{T}_{k} \rightarrow C\left(S^{1}\right) \rightarrow 0
$$

It is well known that two extensions with the same index are isomorphic as $C^{*}$-algebras. (It certainly follows from [V].) We call these algebras $\mathcal{T}$ algebras. It is obvious that $\mathcal{T}_{k}$ is isomorphic to $\mathcal{T}_{-k}$. So we consider only those $\mathcal{T}_{k}$ with $k \geq 0$.

We now give another description of $\mathcal{T}_{k}$ (for $k \geq 0$ ). Let $S_{1}$ be the standard unilateral shift operator acting on the Hilbert space $H=l^{2}$. Then $\mathcal{T}_{1}$ is isomorphic to the universal $C^{*}$-subalgebra of $B(H)$ generated by $S_{1}$. Note that the ideal of the $C^{*}$-subalgebra generated by $1-S_{1} S_{1}^{*}$ is the ideal of compact operators on $H$. We will identify $\mathcal{T}_{1}$ with this algebra whenever it is convenient without further explanation. The element $S_{1}$ in $\mathcal{T}_{1}$ will mean the shift operator. The algebra $\mathcal{T}_{k}(k>0)$ can be identified as follows. Consider the algebra $\mathcal{T}_{1} \otimes M_{k}$, where $M_{k}$ is the $C^{*}$-algebra of $k \times k$ matrix algebra over the complex field. Let $S_{k}=\operatorname{diag}\left(S_{1}, S_{1}, \ldots, S_{1}\right)$ (there are $k$ copies of $S_{1}$ ). Then $\mathcal{T}_{k}$ is isomorphic to the $C^{*}$-subalgebras of $\mathcal{T}_{1} \otimes M_{k}$ generated by $S_{k}$ and $M_{k}(\mathcal{K})$. We will identify these two algebras. Let $e=$ $1-S_{1} S_{1}^{*}$, a minimum projection in $\mathcal{T}_{1}$, and let $\left\{e_{i j}\right\}_{i, j=1}^{k}$ be the matrix unit for $M_{k}$. One checks easily that $\mathcal{T}_{k}$ is generated by $S_{k}$ and $e \otimes e_{i j}$. Note that $1-S_{k} S_{k}^{*}=\sum_{i=1}^{k} e \otimes e_{i i}$. We denote $g_{i j}=e \otimes e_{i j}$. Suppose that $T \in B(H)$ is an isometry and there are $k$ mutually orthogonal equivalent projections $p_{1}, p_{2}, \ldots, p_{k}$ such that $\sum_{i=1}^{k} p_{i}=1-T T^{*}$. Let $a_{i j}$ be the partial isometries such that $a_{i j}^{*} a_{i j}=p_{j}$ and $a_{i j} a_{i j}^{*}=p_{i}$. The $C^{*}$-subalgebra generated by $T$ is isomorphic to $\mathcal{T}_{1}$ and the ideal $J$ of this $C^{*}$-subalgebra generated by $1-T T^{*}$ is isomorphic to $\mathcal{K}$. Note that $a_{11} T=a_{11}\left(1-T T^{*}\right) T=0$ and $\left(T^{m} a_{11}\left(T^{m}\right)^{*}\right) T^{m+l} a_{11}\left(T^{m+l}\right)^{*}=0$ if $l>0$. Let $q_{1}=\sum_{m=1}^{\infty} T^{m} a_{11}\left(T^{m}\right)^{*}$, a projection in $B(H)$. Then $T q_{1}=q_{1} T$. Set $T_{1}=q_{1} T$. Then $T_{1}^{*} T_{1}=q_{1}$ and $1-T_{1} T_{1}^{*}=a_{11}$. Let $B$ be the $C^{*}$-subalgebra generated by $T$ and $\left\{a_{i j}\right\}$ and let $C$ be the $C^{*}$-subalgebra generated by $B$ and $q_{1}, q_{2}, \ldots, q_{k}$, where $q_{i}=$ $\sum_{m=1}^{\infty} T^{m} a_{i i}\left(T^{m}\right)^{*}$. Then $C \cong \mathcal{T}_{1} \otimes M_{k}$. It then follows that $B \cong \mathcal{T}_{k}$. By identifying $B$ with $\mathcal{T}_{k}$, we will say that $\mathcal{T}_{k}$ is generated by $S_{k}$ and $\left\{a_{i j}\right\}$. We would like to point out that the ideal of $B$ generated by $\left\{a_{i j}\right\}$ is not the set of all compact operators on $H$ even if $a_{11}$ is a rank one operator. Let $S_{0}$ be a unitary in $B(H)$ with essential spectrum $S^{1}$. Then $\mathcal{T}_{0}$ is isomorphic to the $C^{*}$-subalgebra of $B(H)$ generated by $S_{0}$ and $\mathcal{K}(H)$. 
In this paper, we will consider direct limits of finite direct sums of matrix algebras over $\mathcal{T}$-algebras.

2.2. Let $k$ be a nonnegative integer. We will determine the $K$-theory of $\mathcal{T}_{k}$. If $k \neq 0$, the index map $\delta: K_{1}\left(C\left(S^{1}\right)\right) \rightarrow K_{0}(\mathcal{K})$ is the multiplication by $-k$. Hence $\delta$ is injective. From the six-term exact sequence in $K$-theory, we obtain:

$$
0 \rightarrow K_{1}\left(M_{n}\left(C\left(S^{1}\right)\right)\right) \rightarrow K_{0}\left(M_{n}(\mathcal{K})\right) \rightarrow K_{0}\left(M_{n}\left(\mathcal{T}_{k}\right)\right) \rightarrow K_{0}\left(M_{n}\left(C\left(S^{1}\right)\right)\right) \rightarrow 0
$$

where $K_{1}\left(M_{n}\left(C\left(S^{1}\right)\right)\right)=\mathbf{Z}, K_{0}\left(M_{n}(\mathcal{K})\right)=\mathbf{Z}$ and $K_{0}\left(M_{n}\left(C\left(S^{1}\right)\right)\right)=\mathbf{Z}$. Therefore,

$$
K_{0}\left(\mathcal{T}_{k}\right)=\mathbf{Z} \oplus \mathbf{Z} / k \mathbf{Z} \quad \text { and } \quad K_{1}\left(\mathcal{T}_{k}\right)=0 .
$$

If $k=0$, then $\delta=0$. The six-term exact sequence breaks into two:

$$
0 \rightarrow K_{0}(\mathcal{K}) \rightarrow K_{0}\left(\mathcal{T}_{0}\right) \rightarrow K_{0}\left(C\left(S^{1}\right)\right) \rightarrow 0
$$

and

$$
0 \leftarrow K_{1}\left(C\left(S^{1}\right)\right) \leftarrow K_{1}\left(\mathcal{T}_{0}\right) \leftarrow 0 .
$$

This implies that

$$
K_{0}\left(\mathcal{T}_{0}\right)=\mathbf{Z} \oplus \mathbf{Z} \quad \text { and } \quad K_{1}\left(\mathcal{T}_{0}\right)=\mathbf{Z} .
$$

We would like to point out that the positive cone of $K_{0}\left(\mathcal{T}_{0}\right)$ is not the usual one on $\mathbf{Z} \oplus \mathbf{Z}$ (cf. 2.3).

2.3. When the stable rank of a $C^{*}$-algebra $A$ is not one, there may be too much information lost in $K_{*}(A)$. Hence, we should also consider $V(A)$, the Murray -von Neumann equivalence classes of projections in matrices over $A$. Note that $V(A)$ is a commutative semigroup.

It is well known and easy to check that if $p \in \mathcal{T}_{1} \backslash \mathcal{K}$ is a projection, then $p$ is equivalent to 1 . From this and the fact that $V\left(C\left(S^{1}\right)\right)=\mathbf{Z}_{+}$, one computes that

$$
V\left(\mathcal{T}_{1}\right)=\mathbf{Z}_{+} \sqcup \mathbf{N}
$$

where the addition in $\mathbf{Z}_{+}$and $\mathbf{N}$ are the usual ones. And if $x \in \mathbf{Z}_{+}, y \in \mathbf{N}$, then $x+y=y$.

For $k>1$, let $S_{k}$ be the isometry in $\mathcal{T}_{k}$ such that $1-S_{k} S_{k}^{*}$ is a projection in $\mathcal{K}$ with rank $k$. Then the $C^{*}$-subalgebra $B$ generated by $S_{k}$ is isomorphic to $\mathcal{T}_{1}$. Note also that the index of every isometry in $\mathcal{T}_{k}$ which is essentially unitary is of multiple $k$. It is then routinely checked that $V\left(\mathcal{T}_{k}\right)$ may be identified with

$$
\mathbf{Z}_{+} \sqcup \mathbf{N} \oplus \mathbf{Z} / k \mathbf{Z}
$$


with the addition defined as follows. Addtions are the usual ones in $\mathbf{Z}_{+}$and $\mathbf{N} \oplus \mathbf{Z} / k \mathbf{Z}$. If $z \in \mathbf{Z}_{+}$and $y \in \mathbf{N} \oplus \mathbf{Z} / k \mathbf{Z}$, then $z+y=y+\bar{z}$, where $\bar{z}$ is the class containing $z$ in $\mathbf{Z} / k \mathbf{Z}$.

When $k=0$, one computes that

$$
V\left(\mathcal{T}_{0}\right)=\left\{(m, n): m \in \mathbf{Z}, n \in \mathbf{Z}_{+}, \quad \text { if } n=0, m \geq 0\right\}
$$

with addition defined in $\mathbf{Z} \oplus \mathbf{Z}$.

We denote $V\left(\mathcal{T}_{k}\right)$ by $F_{k}$. Let $I\left(F_{k}\right)=\mathbf{Z}_{+}$if $k \neq 0$, and $I\left(F_{0}\right)=\{(m, 0)$ : $\left.m \in \mathbf{Z}_{+}\right\}$.

We note that if $p$ and $q$ are two projections in $M_{n}\left(\mathcal{T}_{k}\right)$ such that $[p]=[q]$ in $V\left(\mathcal{T}_{k}\right)$ then there exists an isometry, or a coisometry $W \in M_{n}\left(\mathcal{T}_{k}\right)$ such that $W p W^{*}=q$ and $W^{*} q W=p$.

If $V$ is a semigroup, we will denote $G(V)$ the Grothendieck group of $V$. Let $j_{k}: F_{k} \rightarrow G\left(F_{k}\right)$ be the natural map from $F_{k}$ into $G\left(F_{k}\right)$. In the case that $k=0, F_{0}$ is a subsemigroup of $\mathbf{Z} \oplus \mathbf{Z}$. So $j_{0}$ is injective. For $k>0$, ker $j_{k}=k \mathbf{Z}_{+}$. In both cases, $G\left(F_{k}\right)=K_{0}\left(\mathcal{T}_{k}\right)$. Note that ker $j_{k} \subset I\left(F_{k}\right)$. Denote $Q\left(F_{k}\right)=F_{k} / I\left(F_{k}\right)=j_{k}\left(F_{k}\right) / G\left(j_{k}\left(I\left(F_{k}\right)\right)\right) \cong \mathbf{Z}_{+}$. Let $\eta: F_{k} \rightarrow F_{m}$ be a homomorphism of abelian semigroups, i.e., $\eta$ preserves the additions of the semigroups. A direct computation shows that $\eta$ maps $\operatorname{ker} j_{k}$ into ker $j_{m}$. It follows that $\eta$ gives a group homomorphism from $G\left(F_{k}\right)$ into $G\left(F_{m}\right)$. If $V$ is a direct sum of $F_{k_{i}}, i=1,2, \ldots, n, Q(V)$ is the direct sum of $Q\left(F_{k_{i}}\right), i=$ $1,2, \ldots, n$.

Lemma 2.4. Let $V_{1}$ be a direct sum of $F_{k_{i}} i=1,2, \ldots, n$, let $V_{2}$ be a direct sum of $F_{k_{j}^{\prime}}, j=1,2, . ., l$ and let $\eta: V_{1} \rightarrow V_{2}$ be a homomorphism from semigroup $V_{1}$ into the semigroup $V_{2}$. Then

(a) $\eta$ maps $I\left(V_{1}\right)$ into $I\left(V_{2}\right)$,

(b) $\eta$ induces a quotient map $\bar{\eta}: Q\left(V_{1}\right) \rightarrow Q\left(V_{2}\right)$,

(c) if $\bar{\eta}=0, \eta_{I\left(V_{1}\right)}=0$,

(d) if $V_{1}=F_{k}$ and either $\left.\eta\right|_{I\left(F_{k}\right)}$ or $\bar{\eta}$ is not injective, then $\left.\eta\right|_{I\left(F_{k}\right)}=0$,

(e) if $V_{1}=F_{0}, V_{2}=F_{0}$ and $\eta$ is not injective, then $\left.\eta\right|_{I\left(F_{0}\right)}=0$, and

(f) if $V_{1}=F_{k}$ with $k>0$ and $V_{2}=F_{0}$ then $\left.\eta\right|_{I\left(F_{k}\right)}=0$.

Proof. It is obvious that (b) follows from (a). For (a), it is clear that we only need to show that a homomorphism $\eta: F_{k} \rightarrow F_{m}$ maps $I\left(F_{k}\right)$ into $I\left(F_{m}\right)$.

Case (1): $k=0$ and $m=0$.

Since ker $j_{0}=0$, the homomorphism $\eta$ can be extended to a group homomorphism from $G\left(F_{0}\right)$ into $G\left(F_{0}\right)$. We still use $\eta$ for the extension. Note $G\left(F_{0}\right)=\mathbf{Z} \oplus \mathbf{Z}$. Suppose that $\eta((1,0))=\left(l_{1}, l_{2}\right)$ with $l_{2}>0$. Suppose also that $\eta((0,1))=\left(l_{1}^{\prime}, l_{2}^{\prime}\right)$ with $l_{2}^{\prime}$ nonnegative. Then, for any $n>0$ 
$\eta((-n, 1))=\left(l_{1}^{\prime}-n l_{1}, l_{2}^{\prime}-n l_{2}\right)$. If $n$ is large enough, $l_{2}^{\prime}-n l_{2}<0$. But since $(-n, 1) \in F_{0}, \eta((-n, 1))$ should be in $F_{0}$. This is impossible. Therefore, $l_{2}=0$. Thus $\eta$ maps $I\left(F_{0}\right)$ into $I\left(F_{0}\right)$.

Case (2): $k=0$ and $m>0$.

The map $j_{m} \circ \eta$ extends to a group homomorphism $\tilde{\eta}: G\left(F_{0}\right) \rightarrow G\left(F_{m}\right)(=$ $\mathbf{Z} \oplus \mathbf{Z} / m \mathbf{Z})$. Suppose that

$$
\tilde{\eta}((1,0))=x \oplus \bar{y} \quad \text { and } \quad \tilde{\eta}((0,1))=x_{1} \oplus \bar{y}_{1} .
$$

Then

$$
\tilde{\eta}((-n, 1))=\left(-n x+x_{1}\right) \oplus\left(-n \bar{y}+\bar{y}_{1}\right) .
$$

Since $\tilde{\eta}$ must map $F_{0}$ into $j_{m}\left(F_{m}\right), x$ has to be zero. Since $j_{m}\left(I\left(F_{m}\right)\right)=$ $\mathbf{Z} / m \mathbf{Z}$ and since $\operatorname{ker} j_{m} \subset I\left(F_{m}\right)$, this implies that $\eta$ maps $I\left(F_{0}\right)$ into $I\left(F_{m}\right)$.

Case (3): $k>0$.

Since $\eta$ maps ker $j_{k}$ into ker $j_{m}$, the map $\eta$ gives a group homomorphism $\tilde{\eta}: G\left(F_{k}\right) \rightarrow G\left(F_{m}\right)$. Since $j_{k}\left(I\left(F_{k}\right)\right)$ is torsion, so is $\tilde{\eta}\left(j_{k}\left(I\left(F_{k}\right)\right)\right)$. Therefore $\eta$ maps $I\left(F_{k}\right)$ into $I\left(F_{m}\right)$.

Now for (c), $\bar{\eta}=0$, implies that $\eta$ maps $F_{k}$ into $\mathbf{Z}_{+}$. If $k>0, \eta$ maps ker $j_{k}$ into zero, since $k+(1,0)=(1,0)$ in $F_{k}$ and since the map from $\mathbf{Z}_{+}$ into $G\left(\mathbf{Z}_{+}\right)(=\mathbf{Z})$ is injective. So $\eta$ maps $k \mathbf{Z}_{+}$into zero. The map $\eta$ induces a group homomorphism which maps $\mathbf{Z} \oplus \mathbf{Z} / k \mathbf{Z}$ into $\mathbf{Z}$. Therefore it must map the torsion part to zero. This implies that $\eta$ maps $I\left(V_{1}\right)$ into zero. If $k=0$, let us assume that $\eta((1,0))=l>0$ and $\eta((0,1))=l^{\prime} \geq 0$. Let us use $\eta$ for the extension from $\mathbf{Z} \oplus \mathbf{Z}$ into $\mathbf{Z}$. Then $\eta((-n, 1))=-n l+l^{\prime}$ which is negative if $n$ is large enough. But this is impossible. Therefore $\eta((1,0))=0$. Thus $\eta$ maps $I\left(V_{1}\right)$ into zero.

For (f), by (a), we may write $\eta(x)=k$ and $\eta(y)=(n, z)$, where $x \in$ $I\left(F_{k}\right), k \in I\left(F_{0}\right), y \in \mathbf{N}$ and $z \neq 0$. So $\eta(x)+\eta(y)=(k+n, z) \in F_{0}$. Let $\tilde{\eta}: G\left(F_{k}\right) \rightarrow G\left(F_{0}\right)$ be the group homomorphism induced by $\eta$. Then $\tilde{\eta}(\bar{x})=0$, if $\bar{x} \in \mathbf{Z} / k \mathbf{Z}$. Since $\eta$ and $\tilde{\eta}$ agree on $\mathbf{N} \oplus \mathbf{Z} / k \mathbf{Z}, \eta(\bar{x}+y)=\eta(y)$. So we have $\eta(x+y)=\eta(\bar{x}+y)=\eta(y)=(n, z)$. Therefore $k=0$. This means that $\left.\eta\right|_{I\left(F_{0}\right)}=0$.

To prove (d), we may assume that $V_{2}=F_{m}$. It follows from (a) that $\left.\eta\right|_{I\left(F_{k}\right)}$ is either injective or zero, since $I\left(F_{k}\right) \cong I\left(F_{m}\right) \cong \mathbf{Z}_{+}$. Suppose that $\bar{\eta}$ is not injective. But $Q\left(F_{k}\right) \cong \mathbf{Z}_{+}$and $Q\left(F_{m}\right) \cong \mathbf{Z}_{+}$and $\bar{\eta}$ has to be zero if it is not injective. It follows from (c) that $\left.\eta\right|_{I\left(F_{k}\right)}=0$.

To prove (e), we assume that $\left.\eta\right|_{I\left(F_{0}\right)} \neq 0$. Let $\tilde{\eta}: G\left(F_{0}\right) \rightarrow G\left(F_{0}\right)$ be the group homomorphism induced by $\eta$. Then $\tilde{\eta}$ has to be injective. Consequencely, $\eta$ has to be injective. 
Lemma 2.5. Let $V=\lim \left(V_{n}, \phi_{n, n+1}\right)$, where each $V_{n}$ is a finite direct sum of $F_{k_{i}}^{\prime}$ s. Then there is $n>0$ such that $\phi_{n, \infty}$ is injective on $\phi_{1, n}\left(V_{1}\right)$.

Proof. It is enough to prove the case that $V_{1}=F_{k}$.

We first assume that $k>0$. It is clear that there are only finitely many possible homomorphic images of $F_{k}$, namely, $0, F_{k}, F_{m}$ (where $m \mid k$ ) or $\mathbf{Z}_{+}$. There is an integer $n$ such that the image of $\phi_{1, n}\left(F_{k}\right)$ equals to $\phi_{1, \infty}\left(F_{k}\right)$. If $\phi_{1, \infty}\left(F_{k}\right)=0$, or $=\mathbf{Z}_{+}$, it is clear that $\phi_{n, \infty}$ on $\phi_{1, n}\left(F_{k}\right)$ is injective. Since there are only finitely many images, it is enough to show that any surjective homomorphism $\eta$ from $F_{k}$ (or $F_{m}$ ) onto $F_{k} \quad$ (or $F_{m}$ ) is injective. By (d) of 2.4, both $\left.\eta\right|_{I\left(F_{k}\right)}$ and $\bar{\eta}$ are injective. This immediately implies that $\eta$ maps $I\left(F_{k}\right)$ onto $I\left(F_{k}\right)$ and $\eta(\mathbf{N}) \cap I\left(F_{k}\right)=\{0\}$. Therefore $\eta$ maps $\mathbf{N} \oplus \mathbf{Z} / k \mathbf{Z}$ onto itself. Since the only surjective homomorphism from $\mathbf{Z} \oplus \mathbf{Z} / k \mathbf{Z}$ onto itself is injective, we conlude that $\eta$ has to be injective. This proves the case for $k>0$.

Now we consider the case that $k=0$. There is an integer $n_{1}>0$ such that the image of $\phi_{1, n_{1}}\left(F_{0}\right)$ equals to $\phi_{1, \infty}\left(F_{0}\right)$. If $\phi_{n_{1}, \infty}$ is injective on $\phi_{1, n_{1}}\left(F_{0}\right)$, then we are done. So we assume that it is not injective. Without loss of generality, we may assume that $\phi_{n_{1}, n_{2}}$ on $\phi_{1, n_{1}}\left(F_{0}\right)$ is not injective. Therefore, $\phi_{1, n_{2}}$ is not injective. Let $V_{n_{2}}=B_{1} \oplus B_{2} \oplus \cdots \oplus B_{m}$ and $p_{i}: V_{n_{2}} \rightarrow B_{i}$ be the projection, where each $B_{i} \cong F_{k_{i}}$. We may assume that $B_{1}, B_{2}, \ldots, B_{j} \cong F_{0}$, and $B_{j+k} \cong F_{r_{k}}$ for some $r_{k}>0, k=1,2, \ldots, m-j$. Then every $p_{i} \circ \phi_{1, n_{2}}$ is not injective. By (e) of 2.4, $p_{i} \circ \phi_{1, n_{2}}\left(F_{0}\right)=0$, or $\mathbf{Z}_{+}$. Therefore, there is $n_{3}>0$ such that $\phi_{n_{2}, \infty}$ is injective on $\phi_{n_{2}, n_{3}} \circ p_{i} \circ \phi_{1, n_{2}}\left(F_{0}\right)$ for $i=1,2, \ldots, j$. From the case that $k>0$, we conclude that there is also an integer $n_{4}>0$ such that $\phi_{n_{4}, \infty}$ is injective on each $\phi_{n_{2}, n_{4}}\left(B_{j+k}\right)$. Therefore, there is a large $n>0$ such that $\phi_{n, \infty}$ is injective on $\phi_{1, n}\left(F_{0}\right)$.

2.6. Let $E_{1}$ and $E_{2}$ be two finite direct sums of matrix algebras over $\mathcal{T}$ algebras. Suppose that $E_{1}=\oplus_{j=1}^{L_{1}} M_{l_{j}}\left(\mathcal{T}_{k_{j}}\right)$ and $E_{2}=\oplus_{j=1}^{L_{2}} M_{m_{j}}\left(\mathcal{T}_{r_{j}}\right)$. Each $E_{i}$ is an essential extension

$$
0 \rightarrow I\left(E_{i}\right) \rightarrow E_{i} \rightarrow Q\left(E_{i}\right) \rightarrow 0,
$$

where $I\left(E_{1}\right)=\oplus_{j=1}^{L_{1}} M_{l_{j}}(\mathcal{K}), \quad I\left(E_{2}\right)=\oplus_{j=1}^{L_{2}} M_{m_{j}}(\mathcal{K}), \quad Q\left(E_{1}\right)=$ $\oplus_{j=1}^{L_{1}} M_{l_{j}}\left(C\left(S^{1}\right)\right)$, and $Q\left(E_{2}\right)=\oplus_{j=1}^{L_{2}} M_{m_{j}}\left(C\left(S^{1}\right)\right)$. If $\phi: E_{1} \rightarrow E_{2}$ is a homomorphism, then $\phi$ maps $I\left(E_{1}\right)$ into $I\left(E_{2}\right)$. This happens because $M_{m}\left(C\left(S^{1}\right)\right)$ can not contain any copy of $\mathcal{K}$. Thus we have the following commutative diagram:

$$
\begin{aligned}
0 & \rightarrow I\left(E_{1}\right) \rightarrow E_{1} \rightarrow Q\left(E_{1}\right) \rightarrow 0 \\
\downarrow \phi & \downarrow \phi \quad \downarrow \bar{\phi} \\
0 & \rightarrow I\left(E_{2}\right) \rightarrow E_{2} \rightarrow Q\left(E_{2}\right) \rightarrow 0
\end{aligned}
$$


where $\bar{\phi}: Q\left(E_{1}\right) \rightarrow Q\left(E_{2}\right)$ is the (unique) induced map.

One also has

$$
0 \rightarrow V\left(I\left(E_{i}\right)\right) \rightarrow V\left(E_{i}\right) \rightarrow V\left(Q\left(E_{i}\right)\right) \rightarrow 0 .
$$

The six-term exact sequence of the K-groups of this extension has the form

$$
0 \rightarrow K_{1}\left(E_{i}\right) \rightarrow K_{1}\left(Q\left(E_{i}\right)\right) \rightarrow K_{0}\left(I\left(E_{i}\right)\right) \rightarrow K_{0}\left(E_{i}\right) \rightarrow K_{0}\left(Q\left(E_{i}\right)\right) \rightarrow 0 .
$$

We will denote this exact sequence by $\mathbf{K}\left(E_{i}\right)$. The index map from $K_{1}\left(Q\left(E_{i}\right)\right)$ to $K_{0}\left(I\left(E_{i}\right)\right)$ will be denoted by $\delta_{i}$. By a map from $\mathbf{K}\left(E_{1}\right)$ to $\mathbf{K}\left(E_{2}\right)$ we mean five group homomorphisms $\alpha=\left\{\alpha_{i}\right\}_{i=1}^{5}$ such that the following diagram commutes

$$
\begin{aligned}
& 0 \rightarrow K_{1}\left(E_{1}\right) \rightarrow K_{1}\left(Q\left(E_{1}\right)\right) \rightarrow K_{0}\left(I\left(E_{1}\right)\right) \rightarrow K_{0}\left(E_{1}\right) \rightarrow K_{0}\left(Q\left(E_{1}\right)\right) \rightarrow 0 \\
& \downarrow \alpha_{1} \quad \downarrow \alpha_{2} \quad \downarrow \alpha_{3} \quad \downarrow \alpha_{4} \quad \downarrow \alpha_{5} \\
& 0 \rightarrow K_{1}\left(E_{2}\right) \rightarrow K_{1}\left(Q\left(E_{2}\right)\right) \rightarrow K_{0}\left(I\left(E_{2}\right)\right) \rightarrow K_{0}\left(E_{2}\right) \rightarrow K_{0}\left(Q\left(E_{2}\right)\right) \rightarrow 0 \text {. }
\end{aligned}
$$

Definition 2.7. Recall that an element $s$ in a $C^{*}$-algebra is called hyponormal if $s^{*} s \geq s s^{*}$. For a unital $C^{*}$-algebra $A$, let $S_{n}(A)$ be the set of all nonzero hyponormal partial isometries in $M_{n}(A)$ and $S(A)=\cup_{n=1}^{\infty} S_{n}(A)$ $\left(S_{n}(A) \rightarrow S_{n+1}(A)\right.$ is defined by $\left.s \rightarrow \operatorname{diag}(s, 1)\right)$. Let $S(A)_{0}$ be the set of (nonzero) hyponormal partial isometries which is homotopic to a projection. We denote by $k(A)_{+}=S(A) / S(A)_{0}$. For two hyponormal partial isometries $v_{1}$ and $v_{2}$ in $M_{n}(A),\left[v_{1}\right]=\left[v_{2}\right]$ in $k(A)_{+}$if and only if $\left(1-v_{1}^{*} v_{1}+v_{1}\right)+1_{m}$ is homotopic to $\left(1-v_{2}^{*} v_{2}+v_{2}\right)+1_{m}$ in $S_{m+n}(A)$, where $1_{m}$ is the identity in $M_{m}(A)$. By defining the usual orthogonal addition, $k(A)_{+}$becomes an abelian semigroup. There is a natural embedding $\mathrm{em}: K_{1}(A) \rightarrow k(A)_{+}$. When $A$ has cancellation, $K_{1}(A)=k(A)_{+}$. We think that $k(A)_{+}$may be useful for $C^{*}$-algebra with stable rank other than one.

It is easy to see that $k\left(\mathcal{T}_{0}\right)_{+} \cong K_{1}\left(\mathcal{T}_{0}\right)$. One can also verify that $k\left(M_{m}\left(\mathcal{T}_{k}\right)\right)_{+} \cong \mathbf{Z}_{+}$, if $k>0$. Let $\pi: M_{m}\left(\mathcal{T}_{k}\right) \rightarrow M_{m}\left(C\left(S^{1}\right)\right)$ be the quotient map. Then $\pi$ induces a map from $k\left(M_{m}\left(\mathcal{T}_{k}\right)\right)_{+}$to $k\left(M_{m}\left(C\left(S^{1}\right)\right)\right)_{+} \cong$ $k\left(C\left(S^{1}\right)\right)_{+}$. Clearly, this is an injection. Since $k\left(C\left(S^{1}\right)\right)_{+} \cong K_{1}\left(C\left(S^{1}\right)\right)$, there is an injective homomorphism $\Delta: k\left(\mathcal{T}_{k}\right)_{+} \rightarrow K_{1}\left(C\left(S^{1}\right)\right)$. Note also that $K_{1}\left(M_{m}\left(\mathcal{T}_{k}\right)\right)=\{0\}$, if $k>0$.

2.8. Associate to each $C^{*}$-algebra $A$ a semigroup

$$
\bar{V}(A)=\left\{\left(\left[u^{*} u\right],[u]\right): u \in S(A) \quad \text { and } \quad[u] \in k(A)_{+}\right\}
$$

where $\left[u^{*} u\right]$ is in $V(A)$. Let $d: k(A)_{+} \rightarrow V(A)$ be defined by $d([u])=$ $\left[u^{*} u-u u^{*}\right]$. Let $\left\{u_{t}\right\}$ be a path of hyponormal partial isometries. Then 
$\left\{u_{t}^{*} u_{t}-u_{t} u_{t}^{*}\right\}$ is a path of projections. Thus $d$ is a well defined semigroup homomorphism.

If $E$ is a finite direct sum of matrix algebras over $\mathcal{T}$-algebras, then $d=$ $-\delta \circ \Delta$, where $\delta: K_{1}(Q(E)) \rightarrow K_{0}(I(E))$ is the usual index map.

Definition 2.9. We write the triples

$$
V_{*}(A)=\left\{\left(\left[u^{*} u\right],[u], d([u])\right): u \in S(A) \quad \text { and } \quad[u] \in k(A)_{+}\right\} .
$$

Let $A$ and $B$ be two $C^{*}$-algebras. Suppose that $V_{*}(A)$ and $V_{*}(B)$ are two such triples, then a homomorphism $\eta: V_{*}(A) \rightarrow V_{*}(B)$ is a homomorphisms $\alpha: \bar{V}(A) \rightarrow \bar{V}(B)$ for which

$$
\begin{aligned}
& V(A) \stackrel{d}{\leftarrow} k(A)_{+} \\
& \left.\left.\downarrow \alpha\right|_{V} \quad \downarrow \alpha\right|_{k} \\
& V(B) \stackrel{d}{\leftarrow} k(B)_{+}
\end{aligned}
$$

commutes.

If $\phi: A \rightarrow B$ is a homomorphism, then $\phi$ induces not only a homomorphism from $\bar{V}(A)$ into $\bar{V}(B)$ but also a homomorphism $\phi_{*}: V_{*}(A) \rightarrow V_{*}(B)$. We will use the notation $F_{k *}$ for $V_{*}\left(\mathcal{T}_{k}\right)$.

Let $E$ be a finite direct sum of matrix algebras over $\mathcal{T}$-algebras. It is important to note that the image of $d$ is contained in $I(V(E)) \subset V(E)$.

A fact that we will use later is that any homomorphism from $V_{*}\left(\mathcal{I}_{0}\right)$ into $V_{*}\left(\mathcal{T}_{k}\right)$ with $k>0$ maps the second variable to zero. This is clear, since the only homomorphism from the semigroup $\mathbf{Z}$ into the semigroup $\mathbf{Z}_{+}$is zero.

2.10. Suppose that there is a homomorphism $\eta: V_{*}\left(E_{1}\right) \rightarrow V_{*}\left(E_{2}\right)$ where each $E_{i}$ is a finite direct sum of matrix algebras over $\mathcal{T}$-algebras. It follows from 2.6 that $\eta$ induces the following commutative diagram:

$$
\begin{gathered}
0 \longrightarrow V\left(I\left(E_{1}\right)\right) \longrightarrow V\left(E_{1}\right) \longrightarrow V\left(Q\left(E_{1}\right)\right) \longrightarrow 0 \\
\left.\downarrow \alpha_{3} \longrightarrow \alpha_{4}\right) \quad \downarrow \alpha_{5} \\
0 \longrightarrow V\left(I\left(E_{2}\right)\right) \longrightarrow V\left(E_{2}\right) \longrightarrow V\left(Q\left(E_{2}\right)\right) \longrightarrow 0 .
\end{gathered}
$$

This in turn gives uniquely the following commutative diagram:

$$
\begin{gathered}
K_{0}\left(I\left(E_{1}\right)\right) \longrightarrow K_{0}\left(E_{1}\right) \longrightarrow K_{0}\left(Q\left(E_{1}\right)\right) \longrightarrow 0 \\
\left.\downarrow \alpha_{3}\right) \quad \downarrow \alpha_{4} \quad \begin{array}{l}
\downarrow \alpha_{5} \\
K_{0}\left(I\left(E_{2}\right)\right)
\end{array} \longrightarrow K_{0}\left(E_{2}\right) \longrightarrow K_{0}\left(Q\left(E_{2}\right)\right) \longrightarrow 0 .
\end{gathered}
$$

Furthermore, we have the following commutative diagram:

$$
\begin{aligned}
& 0 \longrightarrow \bar{V}\left(I\left(E_{1}\right)\right) \longrightarrow \bar{V}\left(E_{1}\right) \longrightarrow \bar{V}\left(Q\left(E_{1}\right)\right) \\
& \downarrow \alpha_{3} \quad \downarrow \alpha_{4} \quad \downarrow \alpha_{5} \\
& 0 \longrightarrow \bar{V}\left(I\left(E_{2}\right)\right) \longrightarrow \bar{V}\left(E_{2}\right) \longrightarrow \bar{V}\left(Q\left(E_{2}\right)\right) \text {. }
\end{aligned}
$$


The map $\eta$ also gives a homomorphism from $k\left(E_{1}\right)_{+}$into $k\left(E_{2}\right)_{+}$which, in turn, gives a homomorphism

$$
\alpha_{2}: K_{1}\left(Q\left(E_{1}\right)\right) \rightarrow K_{1}\left(Q\left(E_{2}\right)\right)
$$

Define $\alpha_{1}=\left.\mathrm{em}^{-1} \circ \eta\right|_{\mathrm{em}\left(K_{1}\left(E_{1}\right)\right)} \circ \mathrm{em}: K_{1}\left(E_{1}\right) \rightarrow K_{1}\left(E_{2}\right)$ (note that $\mathrm{em}$ is injective). Note that image of $d$ lies in $I\left(V\left(E_{i}\right)\right)=V\left(I\left(E_{i}\right)\right)$. From the commutative diagram

$$
\begin{aligned}
& V\left(I\left(E_{1}\right)\right) \stackrel{d}{\longleftarrow} k\left(E_{1}\right)_{+} \\
& \left.\left.\downarrow \eta\right|_{V} \quad \downarrow \eta\right|_{k} \\
& V\left(I\left(E_{2}\right)\right) \stackrel{d}{\longleftarrow} k\left(E_{2}\right)_{+}
\end{aligned}
$$

and using the map $\Delta_{i}$ and the natural embedding from $V\left(I\left(E_{i}\right)\right)$ into $K_{0}\left(E_{i}\right)$, we obtain the following commutative diagram:

$$
\begin{aligned}
& 0 \rightarrow K_{1}\left(E_{1}\right) \rightarrow K_{1}\left(Q\left(E_{1}\right)\right) \rightarrow K_{0}\left(I\left(E_{1}\right)\right) \rightarrow K_{0}\left(E_{1}\right) \rightarrow K_{0}\left(Q\left(E_{1}\right)\right) \rightarrow 0 \\
& \downarrow \alpha_{1} \quad \downarrow \alpha_{2} \quad \downarrow \alpha_{3} \quad \downarrow \alpha_{4} \quad \downarrow \alpha_{5} \\
& 0 \rightarrow K_{1}\left(E_{2}\right) \rightarrow K_{1}\left(Q\left(E_{2}\right)\right) \rightarrow K_{0}\left(I\left(E_{2}\right)\right) \rightarrow K_{0}\left(E_{2}\right) \rightarrow K_{0}\left(Q\left(E_{2}\right)\right) \rightarrow 0 \text {. }
\end{aligned}
$$

Set $\alpha=\left\{\alpha_{i}\right\}_{i=1}^{5}$. Then $\alpha$ is a a homomorphism $\alpha: \mathbf{K}\left(E_{1}\right) \rightarrow \mathbf{K}\left(E_{2}\right)$ which is uniquely determined by $\eta$.

Let $E$ be a finite direct sum of matrix algebras over $\mathcal{T}$-algebras. We denote by $\Gamma$ a subset of $K_{0}(Q(E)) \oplus K_{1}(Q(E))$, as follows:

$$
\begin{aligned}
\Gamma & =\left\{\left(\left[u^{*} u\right],[u]\right): u \text { is a normal partial isometry } \in Q(E)\right\} \\
& =\left\{(x, y): x \in V(Q(E)), y \in K_{1}(Q(E)), \text { if } x=0, y=0\right\} .
\end{aligned}
$$

Denote by $K_{*}(Q(E))$ the graded group $K_{0}(Q(E)) \oplus K_{1}(Q(E))$ with the partial order generated by $\Gamma$. It is clear that a homomorphism $\alpha: V_{*}\left(E_{1}\right) \rightarrow$ $V_{*}\left(E_{2}\right)$ also induces a homomorphism $\alpha_{5} \oplus \alpha_{2}: K_{*}\left(Q\left(E_{1}\right)\right) \rightarrow K_{*}\left(Q\left(E_{2}\right)\right)$ (which preserves the order).

From the above we have the following:

Corollary 2.11. Let $E_{1}$ and $E_{2}$ be as in 2.10. Suppose that $\alpha$ is a homomorphism from $V_{*}\left(E_{1}\right)$ to $V_{*}\left(E_{2}\right)$. Then $\alpha$ induces

(i) uniquely two homomorphisms $\gamma$ and $\beta$ such that the following diagram commutes

$$
\begin{aligned}
& 0 \longrightarrow V\left(I\left(E_{1}\right)\right) \longrightarrow V\left(E_{1}\right) \longrightarrow V\left(Q\left(E_{1}\right)\right) \longrightarrow 0 \\
& \downarrow \gamma \quad \downarrow \alpha \quad \downarrow \beta \\
& 0 \longrightarrow V\left(I\left(E_{2}\right)\right) \longrightarrow V\left(E_{2}\right) \longrightarrow V\left(Q\left(E_{2}\right)\right) \longrightarrow 0 \text {, }
\end{aligned}
$$

(ii) uniquely a map $\left\{\alpha_{i}\right\}_{i=1}^{5}$, from $\mathbf{K}\left(E_{1}\right)$ to $\mathbf{K}\left(E_{2}\right)$, and 
(iii) the map $\alpha_{5} \oplus \alpha_{2}$ from $K_{*}\left(Q\left(E_{1}\right)\right)$ to $K_{*}\left(Q\left(E_{2}\right)\right)$ preserves the order.

Lemma 2.12. Let $E_{1}$ and $E_{2}$ be two finite direct sums of matrix algebras over $\mathcal{T}$-algebras such that there is no summand in the sums which is isomorphic to a matrix algebra over $\mathcal{T}_{0}$. Then a homomorphism $\alpha: V\left(E_{1}\right) \rightarrow V\left(E_{2}\right)$ extends to a unique homomorphism $\sigma: V_{*}\left(E_{1}\right) \rightarrow V_{*}\left(E_{2}\right)$. Furthermore, the conclusion of 2.11 holds.

Proof. It follows from the definition that $d$ maps $k\left(\mathcal{T}_{k}\right)_{+}$injectively onto ker $j_{k}$, where $k>0$ and $j_{k}$ is the natural map from $F_{k}$ into $G\left(F_{k}\right)$. We also know that homomorphism from $F_{k}$ into $F_{r}$ (with $r>0$ ) maps ker $j_{k}$ into ker $j_{r}$.

Definition 2.13. Let $V_{*}^{(i)}=\lim _{\rightarrow}\left(V_{n *}^{(i)}, \eta_{n, n+1}^{(i)}\right)$, where $V_{n *}^{(i)}$ is a finite direct

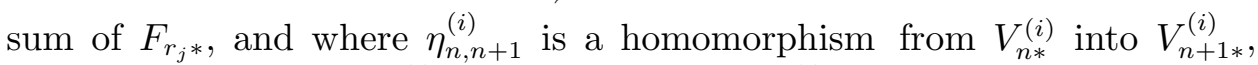
$i=1,2$. Suppose that $V_{n}^{(i)}$ is the first variable, $k_{n}^{(i)}$ is the second variable and $d_{n}^{(i)}$ is the third variable of $V_{n *}^{(i)}$, respectively, for $i=1,2$. Let $V^{(i)}=\lim _{\rightarrow} V_{n}^{(i)}$, $k=\lim _{\rightarrow} k_{n}^{(i)}, d^{(i)}=\lim _{\rightarrow} d_{n}^{(i)}$ and $\bar{V}^{(i)}=\lim _{\rightarrow}\left(\bar{V}_{n}^{(i)}, \eta_{n, n+1}^{(i)}\right)$. A homomorphism $\phi: V_{*}^{(1)} \rightarrow V_{*}^{(2)}$ is a homomorphism $\alpha: \vec{V}^{(1)} \rightarrow \bar{V}^{(2)}$ for which

$$
\begin{array}{ccc}
V^{(1)} & \stackrel{d^{(1)}}{\leftarrow} & k^{(1)} \\
\left.\downarrow \alpha\right|_{V^{(1)}} & \left.\downarrow \alpha\right|_{k(A)_{+}} \\
V^{(2)} & \stackrel{d^{(2)}}{\leftarrow} & k^{(2)}
\end{array}
$$

is commutative.

Let $E=\lim _{\rightarrow}\left(E_{i}, \phi_{i, i+1}\right)$ be a direct limit of finite direct sums of matrix algebras over $\overrightarrow{\mathcal{T}}$-algebras and let $I(E)=\lim \left(I\left(E_{i}\right), \phi_{i, i+1}\right)$. Then $I(E)$ is an ideal of $E$. Set $Q(E)=E / I(E)$. Then it is clear that $Q(E)=$ $\lim \left(Q\left(E_{i}\right), \bar{\phi}_{i, i+1}\right)$, where $\bar{\phi}_{i, i+1}$ is the map induced by $\phi$ (see 2.6).

Lemma 2.14. Let $E=\lim \left(E_{i}, \phi_{i, i+1}\right)$ and $E^{\prime}=\lim \left(E_{i}^{\prime}, \psi_{i, i+1}\right)$ be two inductive limit $C^{*}$-algebras where each $E_{i}$ or each $E_{i}^{\prime} \overrightarrow{i s}$ a finite direct sum of matrix algebras over $\mathcal{T}$-algebras. Suppose that each connecting map in the two inductive limit systems satisfies the following condition: any partial map from $M_{n}\left(\mathcal{T}_{0}\right)$ to another algebra vanishes on $M_{n}(\mathcal{K})$. Let $\alpha$ be a homomorphism from $V_{*}(E)$ to $V_{*}\left(E^{\prime}\right)$. Then $\alpha$ induces

(i) uniquely two homomorphisms $\gamma$ and $\beta$ such that the following diagram commutes

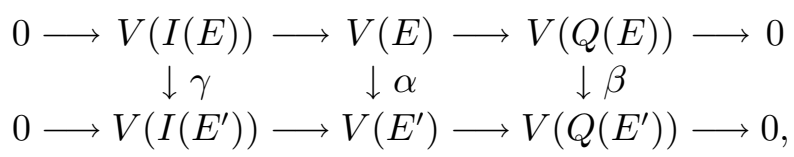


(ii) $\quad \alpha$ induces uniquely a map $\left\{\alpha_{i}\right\}_{i=1}^{5}$ from $\mathbf{K}(E)$ to $\mathbf{K}\left(E^{\prime}\right)$.

Furthermore, $\alpha_{5} \oplus \alpha_{2}$ from $K_{*}(Q(E))$ to $K_{*}\left(Q\left(E^{\prime}\right)\right)$ preserves the order. In particular, if $\alpha$ is an isomorphism, all the maps induced by $\alpha$ are isomorphisms.

Proof. Let $V_{*}(E)=\lim _{\rightarrow}\left(V_{*}\left(E_{n}\right), \eta_{n, n+1}\right)$ and $V_{*}\left(E^{\prime}\right)=\lim _{\rightarrow}\left(V_{*}\left(E_{n}^{\prime}\right), \eta_{n, n+1}^{\prime}\right)$. There exists $n_{1}^{\prime}>1 \mathrm{such}$ that $\alpha\left(\eta_{1, \infty}\left(\bar{V}\left(E_{1}\right)\right)\right) \subset \eta_{n_{1}^{\prime}, \infty}\left(\vec{V}\left(E_{n_{1}^{\prime}}^{\prime}\right)\right)$. To see this, it is enough to assume that $E_{1}=M_{n}\left(\mathcal{T}_{k}\right)$. If $k>0, \bar{V}\left(E_{1}\right)$ is finitely generated. If $k=0$, although $\bar{V}\left(E_{1}\right)$ is not finitely generated, the connecting map $\bar{V}\left(E_{1}\right) \rightarrow \bar{V}\left(E_{2}\right)$ vanishes on $\bar{V}\left(I\left(E_{1}\right)\right)$. The image in $\bar{V}\left(E_{2}\right)$ is generated by a single element. In both cases, the inclusion follows. There is $n_{1}^{\prime \prime}$ such that $\eta_{n_{1}^{\prime \prime}, m}$ maps $\eta_{n_{1}^{\prime \prime}, n_{1}^{\prime}}\left(V\left(E_{n_{1}^{\prime}}^{\prime}\right)\right)$ injectively into $V\left(E_{m}\right)$ for any $m>n_{1}^{\prime \prime}$. This is possible because of 2.5. Choose $n_{1}=n_{1}^{\prime \prime}+1$. Let $h$ be the inverse of $\eta_{n_{1}, \infty}^{\prime}$ on $\eta_{n_{1}, \infty}^{\prime}\left(\eta_{n_{1}^{\prime}, n_{1}}\left(\bar{V}\left(E_{n_{1}^{\prime}}^{\prime}\right)\right)\right)$. Define

$$
\alpha^{(1)}=\eta_{n_{1}^{\prime}, n_{1}}^{\prime} \circ h \circ \alpha \circ \eta_{1, \infty} .
$$

Then the following diagram commutes:

$$
\begin{aligned}
& \bar{V}\left(E_{1}\right) \longrightarrow \bar{V}(E) \\
& \downarrow \alpha^{(1)} \quad \downarrow \alpha \\
& \bar{V}\left(E_{n_{1}}^{\prime}\right) \longrightarrow \bar{V}\left(E^{\prime}\right) \text {. }
\end{aligned}
$$

Since $k\left(E_{1}\right)_{+}$is finitely generated, we can choose $\alpha^{(1)}$ so that the following diagram commutes:

$$
\begin{array}{cc}
V_{*}\left(E_{1}\right) & \longrightarrow V_{*}(E) \\
\downarrow \alpha^{(1)} & \downarrow \alpha \\
V_{*}\left(E_{n_{1}}^{\prime}\right) \longrightarrow & V_{*}\left(E^{\prime}\right) .
\end{array}
$$

As above, there is $n_{2}^{\prime}$ such that

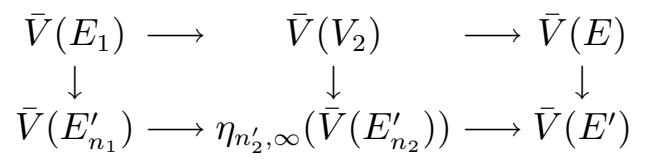

commutes. The above argument actually says that one has the following commutative diagram

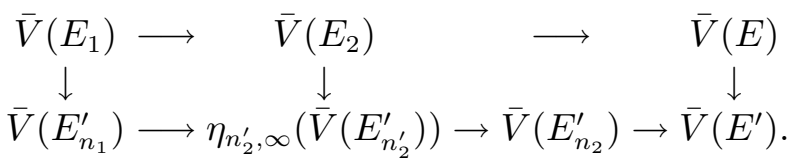

This will give the following commutative diagram: 


$$
\begin{aligned}
& \bar{V}\left(E_{1}\right) \longrightarrow \bar{V}\left(E_{2}\right) \longrightarrow \bar{V}(E) \\
& \downarrow \alpha^{(1)} \quad \downarrow \alpha^{(2)} \quad \downarrow \alpha \\
& \bar{V}\left(E_{n_{1}}^{\prime}\right) \longrightarrow \bar{V}\left(E_{n_{2}}^{\prime}\right) \longrightarrow \bar{V}\left(E^{\prime}\right) \text {. }
\end{aligned}
$$

The above argument further gives us the following diagram:

$$
\begin{array}{ccc}
V_{*}\left(E_{1}\right) & \longrightarrow V_{*}\left(E_{2}\right) & \longrightarrow V_{*}(E) \\
\downarrow \alpha^{(1)} & \downarrow \alpha^{(2)} & \downarrow \alpha \\
V_{*}\left(E_{n_{1}}^{\prime}\right) & \longrightarrow V_{*}\left(E_{n_{2}}^{\prime}\right) \longrightarrow V_{*}\left(E^{\prime}\right) .
\end{array}
$$

Continuing this way, we have a commutative diagram:

$$
\begin{aligned}
& V_{*}\left(E_{1}\right) \longrightarrow V_{*}\left(E_{2}\right) \longrightarrow \cdots \longrightarrow V_{*}(E) \\
& \downarrow \alpha^{(1)} \quad \downarrow \alpha^{(2)} \quad \downarrow \alpha \\
& V_{*}\left(E_{n_{1}}^{\prime}\right) \longrightarrow V_{*}\left(E_{n_{2}}^{\prime}\right) \longrightarrow \cdots \longrightarrow V_{*}\left(E^{\prime}\right)
\end{aligned}
$$

where each map is homomorphism. We will call this diagram (1).

If $\alpha$ is an isomorphism, by the similar argument as above, by choosing $m_{2}$ first (after choosing $n_{1}$ ), then choosing $n_{2}$, one has the following commutative diagram:

$$
\begin{aligned}
& V_{*}\left(E_{1}\right) \longrightarrow V_{*}\left(E_{m_{2}}\right) \longrightarrow \cdots \longrightarrow V_{*}(E) \\
& \downarrow \alpha^{(1)} \nearrow \alpha^{(1)^{\prime}} \downarrow \alpha^{(2)} \quad \nearrow \alpha^{(2)^{\prime}} \quad \alpha \downarrow \uparrow \beta \\
& V_{*}\left(E_{n_{1}}^{\prime}\right) \quad \longrightarrow V_{*}\left(E_{n_{2}}^{\prime}\right) \quad \longrightarrow \quad \cdots \longrightarrow V_{*}\left(E^{\prime}\right) \text {. }
\end{aligned}
$$

We will call this diagram (2).

By passing to a subsequence and changing the notation, we may assume that $n_{k}=k$ and $m_{k}=k$ in both diagram (1) and diagram (2).

For each $k$, diagram (1) gives the following commutative diagram:

$$
\begin{gathered}
0 \longrightarrow V\left(I\left(E_{k}\right)\right) \longrightarrow V\left(E_{k}\right) \longrightarrow V\left(Q\left(E_{k}\right)\right) \longrightarrow 0 \\
\left.\downarrow \gamma^{(k)} \begin{array}{lc}
\downarrow \alpha^{(k)} & \downarrow \beta^{(k)}
\end{array}\right) \\
0 \rightarrow V\left(I\left(E_{k}^{\prime}\right)\right) \longrightarrow V\left(E_{k}^{\prime}\right) \longrightarrow V\left(Q\left(E_{k}^{\prime}\right)\right) \longrightarrow 0 .
\end{gathered}
$$

It also gives commutative diagrams

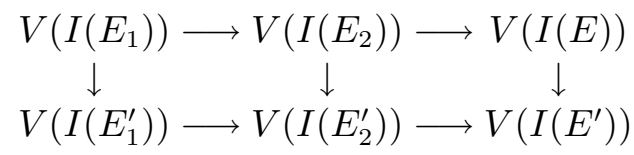

and

$$
\begin{aligned}
& V\left(Q\left(E_{1}\right)\right) \longrightarrow V\left(Q\left(E_{2}\right)\right) \longrightarrow V(Q(E)) \\
& V\left(Q\left(E_{1}^{\prime}\right)\right) \longrightarrow V\left(Q\left(E_{2}^{\prime}\right)\right) \longrightarrow V\left(Q\left(E^{\prime}\right)\right)
\end{aligned}
$$


Combining these commutative diagrams, we obtain the following commutative diagram

$$
\begin{gathered}
0 \longrightarrow V(I(E)) \\
\downarrow \gamma(E) \longrightarrow V(Q(E)) \longrightarrow 0 \\
0 \rightarrow V\left(I\left(E^{\prime}\right)\right) \longrightarrow V\left(E^{\prime}\right) \longrightarrow V\left(Q\left(E^{\prime}\right)\right) \longrightarrow 0 .
\end{gathered}
$$

Furthermore, if $\alpha$ is an isomorphism, then diagram (2) ensures that $\alpha, \beta$ and $\gamma$ are invertible.

By 2.11 and diagram (1), we obtain the following commutative diagram

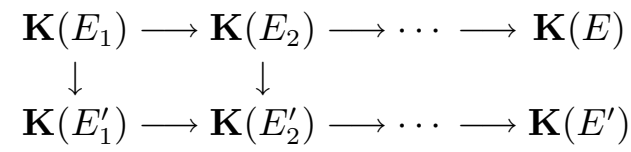

which will induces a map from $\mathbf{K}(H)$ to $\mathbf{K}\left(E^{\prime}\right)$. It is clear that

$$
K_{*}(Q(E))=\lim _{\rightarrow} K_{*}\left(Q\left(E_{n}\right)\right) \quad \text { and } \quad K_{*}\left(Q\left(E^{\prime}\right)\right)=\lim _{\rightarrow} K_{*}\left(E_{n}\right) .
$$

Thus we also have that $\alpha_{5} \oplus \alpha_{2}$ preserves the order.

If $\alpha$ is a isomorphism, from diagram (2), we have

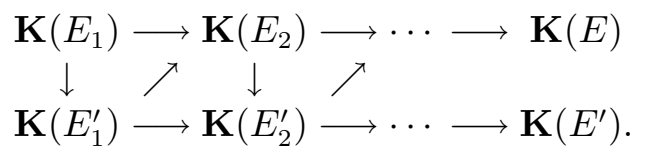

This gives an isomorphism from $\mathbf{K}(E)$ onto $\mathbf{K}\left(E^{\prime}\right)$. In particular, $\alpha_{i}$ is isomorphism, $i=1,2, \ldots, 5$.

Corollary 2.15. Let $E$ and $E^{\prime}$ be as in Lemma 2.13 with additional assumption that no $\mathcal{T}_{0}$ ever appears in $E_{i}$ and $E_{i}^{\prime}$. Let $\alpha$ be a homomorphism from $V(E)$ into $V\left(E^{\prime}\right)$. Then all conclusions in Lemma 2.13 hold.

\section{Existence.}

In this section, we first prove an existence Theorem (3.1). Then we show another type of existence Theorem (3.7), which says that given any possible invariant $V_{*}$, there is an inductive limit $A$ of finite direct sums of matrix algebras over $\mathcal{T}$-algebras of real rank zero such that $V_{*}(A)=V_{*}$. We also show that every real rank zero $A \mathbb{T}$ algebra is an inductive limit of finite direct sums of matrix algebras over $\mathcal{T}$-algebras, i.e., an $A \mathcal{T}$-algebra.

If $\phi: A \rightarrow B$ is a homomorphism from $C^{*}$-algebra $A$ into $C^{*}$-algebra $B$, we will use the notation $\phi_{* 0}$ and $\phi_{* 1}$ for the maps from $K_{0}(A)$ into $K_{0}(B)$ and from $K_{1}(A)$ into $K_{1}(B)$, respectively. 
Theorem 3.1. Let $E_{1}$ and $E_{2}$ be two finite direct sums of matrix algebras over $\mathcal{T}$-algebras. Suppose that there is a homomorphism $\alpha$ from $V_{*}\left(E_{1}\right)$ into $V_{*}\left(E_{2}\right)$ which maps $\left[1_{E_{1}}\right]$ into $[P]$ for some projection $P$ in $E_{2}$. Then

(i) $\alpha$ induces two homomorphisms $\gamma$ and $\beta$ such that the following diagram commutes:

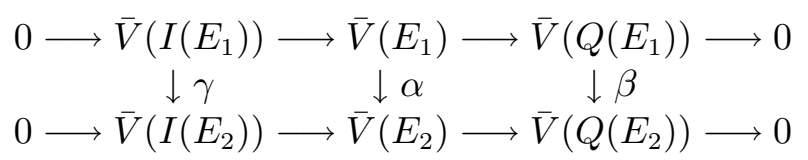

(ii) $\alpha$ induces a map, consisting of five group homomorphisms $\left\{\alpha_{i}\right\}_{i=1}^{5}$, from $\mathbf{K}\left(E_{1}\right)$ to $\mathbf{K}\left(E_{2}\right)$.

Suppose further that $\psi$ is a *-homomorphism from $Q\left(E_{1}\right)$ to $Q\left(E_{2}\right)$ with $\psi_{* 0}=\alpha_{5}$ and $\psi_{* 1}=\alpha_{2}$ which satisfies the following condition: if $M_{m}\left(\mathcal{T}_{0}\right)$ is a summand of $E_{1}$ and $M_{m^{\prime}}\left(\mathcal{T}_{k}\right)$ is a summand of $E_{2}$ and the partial map of $\gamma$ from $I\left(F_{0}\right)$ into $I\left(F_{k}\right)$ is not zero, then the partial map of $\psi$ from $Q\left(M_{m}\left(\mathcal{T}_{0}\right)\right)$ to $Q\left(M_{m^{\prime}}\left(\mathcal{T}_{k}\right)\right)$ is injective. Then there exists a homomorphism $\phi$ from $E_{1}$ to $E_{2}$ such that $\phi$ induces $\alpha, \phi\left(1_{E_{1}}\right)=P$ and such that $\pi_{2} \circ \phi=\psi \circ \pi_{1}$, where $\pi_{i}$ is the quotient map from $E_{i}$ to $Q\left(E_{i}\right)$.

Proof. First, we note from Corollary 2.11 that the commuting diagrams in the theorem comes automatically from map $\alpha$. Also if $\phi$ is a homomorphism from $E_{1}$ into $E_{2}$ such that $\phi$ induces $\alpha$ from $V_{*}\left(E_{1}\right)$ to $V_{*}\left(E_{2}\right)$ and such that $\pi_{2} \circ \phi=\psi \circ \pi_{1}$, then $\phi$ induces $\psi$. Clearly, we may assume that $E_{2}$ has only one summand, say $M_{m}\left(\mathcal{I}_{r}\right)$.

Write $E_{1}=\oplus_{j=1}^{k} E_{1}^{(j)}$ where $E_{1}^{(j)}=M_{n_{j}}\left(\mathcal{T}_{k_{j}}\right)$ and let $1_{n_{j}}$ be the unit of $E_{1}^{(j)}$. Associated with each $E_{1}^{(j)}$ we have an extension

$$
0 \rightarrow I\left(E_{1}^{(j)}\right) \rightarrow E_{1}^{(j)} \rightarrow Q\left(E_{1}^{(j)}\right) \rightarrow 0 .
$$

Write $\psi=\oplus_{i=1}^{k} \psi_{i}$ where each $\psi_{i}$ is a homomorphism from $M_{n_{i}}\left(C\left(S^{1}\right)\right)$ into $M_{m}\left(S^{1}\right)$. There exists a unitary $V \in M_{m}\left(C\left(S^{1}\right)\right)$ such that $\operatorname{Ad} V \circ \psi=$ $\operatorname{diag}\left(\psi_{1}, \cdots, \psi_{k}\right)$. Let $W=\operatorname{diag}\left(W_{1}, \cdots, W_{k}\right)$ be a unitary in $M_{m}\left(C\left(S^{1}\right)\right)$. Then $\operatorname{Ad}(W V) \circ \psi$ still has diagonal form. We can choose $W$ such that $W V$ can be lifted to a unitary in $E_{2}$. So we may assume that $\psi$ already is of diagonal form:

$$
\psi=\operatorname{diag}\left(\psi_{1}, \cdots, \psi_{k}\right) .
$$

Let $e_{i}=\operatorname{diag}\left(0, \cdots, \psi_{i}\left(1_{n_{i}}\right), 0, \cdots, 0\right)$. If $\left[e_{i}\right]=m_{i}$, then $m \geq m_{1}+m_{2}+$ $\cdots+m_{k}$. Notice that $e_{i} M_{m}\left(C\left(S^{1}\right)\right) e_{i} \cong M_{m_{i}}\left(C\left(S^{1}\right)\right)$. We note that some of $\psi_{j}$ may be zero. This means that the corresponding $m_{j}=0$.

Since $\alpha\left(\sum_{i=1}^{k}\left[1_{n_{i}}\right]\right)=[P]$, there are mutually orthogonal projections $d_{1}$, $d_{2}, \ldots, d_{k}$ in $P M_{m}\left(\mathcal{T}_{r}\right) P$ such that $P=\sum_{i=1}^{k} d_{i}, \alpha\left(\left[1_{n_{j}}\right]\right)=\left[d_{j}\right]$ and $\pi_{2}\left(d_{j}\right)=$ 
$\psi\left(1_{n_{j}}\right)$. Note that

$$
\pi\left(d_{i}\right) M_{m}\left(C\left(S^{1}\right)\right) \pi\left(d_{i}\right) \cong M_{m_{i}}\left(C\left(S^{1}\right)\right)
$$

if $d_{i}$ is not in $M_{m}(\mathcal{K})$. Let $\psi_{j}=\left.\psi\right|_{Q\left(E_{1}^{(j)}\right)}$. It is enough to show that, for each $j$, there exists a unital homomorphism $\phi_{j}: E_{1}^{(j)} \rightarrow d_{j} M_{m}\left(\mathcal{T}_{r}\right) d_{j}$ such that $\pi_{2} \circ \phi_{j}=\psi_{j} \circ \pi_{1}$ and the homomorphism from $V\left(E_{1}^{(j)}\right)$ into $V\left(E_{2}\right)$ induced by $\phi_{j}$ agrees with $\left.\alpha\right|_{V\left(E_{1}^{(j)}\right)}$.

We first consider the case that $d_{j} \in M_{m}(\mathcal{K})$. Let $\left[d_{j}\right]=b_{j} \in \mathbf{Z}_{+} \backslash\{0\}$ (if $b_{j}=0$, we define $\phi_{j}=0$ ). So $d_{i} M_{m}(\mathcal{K}) d_{i} \cong M_{b_{i}}$. Since $\alpha$ preserves the addition, $n_{i}$ divides $b_{i}$. Let $\Phi$ be the quotient map from $M_{n_{j}}\left(\mathcal{T}_{k_{j}}\right)$ onto $M_{n_{j}}$. Since $n_{i}$ divides $b_{i}$, there is a unital homomorphism $\Psi$ from $M_{n_{j}}$ into $d_{j} M(\mathcal{K}) d_{j}$. We define $\phi_{j}=\Psi \circ \Phi$. It is worthy of pointing out that, in this case, $\phi_{j}$ vanishes on $I\left(E_{1}^{(j)}\right)$ and $\psi_{j}=0$.

Now the homomorphism $\phi_{j}$ maps $E_{1}^{(j)}$ to $d_{j} E_{2} d_{j}$ and sends the identity to $d_{j}$. Furthermore, $\phi_{j}$ induces a map from $V\left(E_{1}^{(j)}\right)$ to $V\left(E_{2}\right)$ which agrees $\alpha$ on $V\left(E_{1}^{(j)}\right)$.

Let us now assume that $d_{j}$ is not in $M_{m}(\mathcal{K})$. Let $\left\{e_{i l}\right\}$ be a matrix unit for $M_{n_{j}}(\mathbf{C})$ which is in $M_{n_{j}}\left(\mathcal{T}_{k_{j}}\right)$. Let $\psi_{j}\left(\pi\left(e_{i l}\right)\right)=\bar{q}_{i l}$. Then $\bar{q}_{i l}$ is a matrix unit (for $M_{n_{j}}$ ). There is a matrix unit $\left\{q_{i l}^{\prime}\right\} \in d_{j} M_{m}\left(\mathcal{T}_{k}\right) d_{j}$ such that $\pi\left(q_{i l}^{\prime}\right)=$ $\bar{q}_{i l}$ (see $[$ Eff $\left.]\right)$. Note $\alpha\left(\left[e_{i i}\right]\right)=\alpha\left(\left[e_{11}\right]\right),\left[q_{i i}^{\prime}\right]=\left[q_{11}^{\prime}\right]$ in $V\left(d_{j} M_{m}\left(\mathcal{T}_{k}\right) d_{j}\right)$ and $\alpha_{5}\left(\left[e_{11}\right]\right)=\left[\bar{q}_{11}^{\prime}\right]$. There is a projection $q^{\prime} \in d_{j} M_{m}\left(\mathcal{T}_{k}\right) d_{j}$ such that $\alpha\left(\left[e_{11}\right]\right)=\left[q^{\prime}\right]$ in $V\left(d_{j} M_{m}\left(\mathcal{T}_{k}\right) d_{j}\right)$. Then either $\left[q^{\prime}\right]-\left[q_{11}^{\prime}\right]$, or $\left[q_{11}^{\prime}\right]-\left[q^{\prime}\right]$ is in $I\left(V\left(d_{j} M_{m}\left(\mathcal{T}_{k}\right) d_{j}\right)\right) \cong \mathbf{Z}_{+}$. Since $\sum_{i=1}^{n_{j}} \alpha\left(\left[e_{i i}\right]\right)=\left[d_{j}\right]$, the second case will not happen. Let $d_{j}^{\prime}=d_{j}-\sum_{i=1}^{n_{j}} q_{i i}^{\prime}$. Then $\left[d_{j}^{\prime}\right]=n_{j}\left[q^{\prime}\right]-n_{j}\left[q_{11}^{\prime}\right]=n_{j}\left(\left[q^{\prime}\right]-\left[q_{11}^{\prime}\right]\right) \geq$ 0 . If $\left[q^{\prime}\right]-\left[q_{11}^{\prime}\right] \neq 0$, there are mutually orthogonal and mutually equivalent projections $a_{i}$ in $d_{j}^{\prime} M_{m}(\mathcal{K}) d_{j}^{\prime}$ such that $\left[a_{i}\right]=\left[q^{\prime}\right]-\left[q_{11}^{\prime}\right]$ and $\sum_{i=1}^{n_{j}} a_{i}=d_{j}^{\prime}$. There is a matrix unit $\left\{a_{i l}\right\} \subset d_{j}^{\prime} M_{m}(\mathcal{K}) d_{j}^{\prime}$ such that $a_{i i}=a_{i}, i=1,2, \ldots, n_{j}$. Let $q_{i l}=q_{i l}^{\prime}+a_{i l}$. Then $\pi\left(q_{i l}\right)=\bar{q}_{i l}$ and $\alpha\left(\left[e_{i i}\right]\right)=\left[q_{i i}\right]$.

Let $g: M_{n_{j}}(\mathbf{C}) \rightarrow d_{j} M_{m}\left(\mathcal{T}_{r}\right) d_{j}$ be the monomorphism which maps $e_{i l}$ into $q_{i l}$. Let $\bar{g}=\pi_{2} \circ g$. Then we can write

$$
\psi_{j}=\bar{g} \otimes \tilde{\psi}_{j},
$$

where $\tilde{\psi}_{j}$ is a unital homomorphism from $C\left(S^{1}\right)$ into $\pi_{2}\left(q_{11}\right) Q\left(M_{m}\left(\mathcal{T}_{r}\right)\right) \pi_{2}\left(q_{11}\right) \cong M_{t}\left(C\left(S^{1}\right)\right)$ and $t=m_{j} / n_{j}$. It is enough to show that there exists a homomorphism $\tilde{\phi}_{j}: e_{11} M_{n_{j}}\left(\mathcal{I}_{k_{j}}\right) e_{11} \rightarrow g\left(e_{11}\right) M_{m}\left(\mathcal{I}_{r}\right) g\left(e_{11}\right)$ such that $\pi_{2} \circ \tilde{\phi}_{j}\left(S_{k_{j}}\right)=\psi_{j}(z)$ where $z$ is the canonical generator of $C\left(S^{1}\right)$ and where $S_{k_{j}}$ is the canonical isometry of $\mathcal{T}_{k_{j}}$ and such that the map from $V_{*}\left(e_{11} M_{n_{j}}\left(\mathcal{T}_{k_{j}}\right) e_{11}\right)$ into $V_{*}\left(g\left(e_{11}\right) M_{m}\left(\mathcal{T}_{r}\right) g\left(e_{11}\right)\right)$ agrees with the restriction of $\alpha$. Note that $\pi_{1}\left(e_{11} M_{n_{j}}\left(\mathcal{T}_{k_{j}}\right) e_{11}\right) \cong C\left(S^{1}\right)$. 
Case (1): $r=0$.

First, consider the case $k_{j}=0$. Note that $e_{11} M_{n_{j}}\left(\mathcal{T}_{0}\right) e_{11} \cong \mathcal{T}_{0}$.

Since $r=0$, every normal partial isometry in $M_{m}\left(C\left(S^{1}\right)\right)$ lifts to a normal partial isometry in $M_{m}\left(\mathcal{T}_{0}\right)$. In particular, $\psi_{j}(z)$ lifts to a unitary $W \in g\left(e_{11}\right) M_{m}\left(\mathcal{T}_{r}\right) g\left(e_{11}\right)$. Let $A$ be the $C^{*}$-algebra generated by $W$ and $g\left(e_{11}\right) M_{m}(\mathcal{K}) g\left(e_{11}\right)$. Then $A \cong \mathcal{T}_{0}$. Note that $W$ has essential spectrum $S^{1}$.

Suppose that $\gamma$ has multiplicity $l_{1}>0$. Embedding $A$ into $B\left(l^{2}\right)$ such that $I(A)=I\left(\mathcal{T}_{0}\right)$ maps onto $\mathcal{K}\left(l^{2}\right)$. Let $\lambda_{1}$ be an isomorphism from $M_{l_{1}}(B)$ onto $B\left(l^{2}\right)$. Let $\lambda_{2}: \mathcal{T}_{0} \rightarrow M_{l_{1}}\left(B\left(l^{2}\right)\right)$ be an amplification such that $\lambda_{2}(a)=$ $\operatorname{diag}(a, a, \ldots, a)$ (there are $l_{1}$ copies of $a$ ). Set $\phi^{\prime}=\lambda_{1} \circ \lambda_{2}$. Then $\left(\right.$ in $B\left(l^{2}\right)$ ) both unitaries $\phi^{\prime}\left(S_{0}\right)$ and $W$ have the same essential spectrum $S^{1}$, i.e., spectrum of $\pi\left(\phi^{\prime}\left(S_{0}\right)\right)=\psi(z)=S^{1}$. Therefore there is a unitary $U \in B\left(l^{2}\right)$ such that $U^{*} \phi^{\prime}\left(S_{0}\right) U-W \in \mathcal{K}\left(l^{2}\right)$. Thus $U^{*} \phi^{\prime}\left(S_{0}\right) U \in A$. We now define $\tilde{\phi}_{j}(a)=U^{*} \phi^{\prime}(a) U$ for $a \in \mathcal{T}_{0}$. It is easy to see that $\tilde{\phi}$ meets the requirement in this case.

In the case that $\gamma=0$ (i.e. $\alpha_{3}=0$ ), let

$$
f: M_{t}\left(C\left(S^{1}\right)\right) \rightarrow g\left(e_{11}\right) M_{m}\left(\mathcal{T}_{r}\right) g\left(e_{11}\right)
$$

be a monomorphism which splits the short exact sequence:

$$
0 \rightarrow g\left(e_{11}\right) M_{m}(\mathcal{K}) g\left(e_{11}\right) \rightarrow g\left(e_{11}\right) M_{m}\left(\mathcal{T}_{0}\right) g\left(e_{11}\right) \rightarrow M_{t}\left(C\left(S^{1}\right)\right) \rightarrow 0 .
$$

We define

$$
\tilde{\phi}_{j}=f \circ \psi_{j} \circ \pi
$$

Clearly $\tilde{\phi}_{j}$ induces the same map from $V_{*}\left(e_{11} M_{n_{j}}\left(\mathcal{T}_{k_{j}}\right) e_{11}\right)$ into $V_{*}\left(g\left(e_{11}\right) M_{m}\left(\mathcal{T}_{r}\right) g\left(e_{11}\right)\right)$ as the restriction of $\alpha$.

If $k_{j}>0$, we also define

$$
\tilde{\phi}_{j}=f \circ \psi_{j} \circ \pi
$$

To see that $\tilde{\phi}_{j}$ meets other requirements, we only need to show that $\alpha$ maps $I\left(F_{k_{j}}\right)$ into zero. The homomorphism $\alpha$ maps ker $j_{k_{j}}$ into zero, since the map $j_{0}: F_{0} \rightarrow G\left(F_{0}\right)$ is injective. Thus $\alpha$ is not injective. By part (d) of 2.4, $\alpha$ indeed maps $I\left(F_{k_{j}}\right)$ into zero.

Case (2): $r>0$. Note that $\tilde{\psi}_{j}(z)$ can be lifted to an isometry $W$ in $g\left(e_{11}\right) M_{m}\left(\mathcal{T}_{r}\right) g\left(e_{11}\right)$. From the second diagram, one sees that

$$
\alpha\left(\left[1-S_{k_{j}} S_{k_{j}}^{*}\right]\right)=\alpha_{3}\left(\left[1-S_{k_{j}} S_{k_{j}}^{*}\right]\right)=\left[g\left(e_{11}\right)-W W^{*}\right] .
$$

We first consider the case that $\alpha\left(\left[1-S_{k_{j}} S_{k_{j}}^{*}\right]\right)=0$. In this case, we may assume that $W$ is a unitary. 
Let us first consider the case that $k_{j}=0$ and $\gamma$ does not map $I\left(F_{k_{j}}\right)$ into zero. By the assumption on $\psi$, the essential spectrum of $W$ is the whole circle. In particular $s p(W)=S^{1}$.

Denote by $B$ the $C^{*}$-subalgebra generated by $W$ and $g\left(e_{11}\right) I\left(M_{n_{j}}\left(\mathcal{T}_{r}\right)\right) g\left(e_{11}\right) \cong \mathcal{K}$. Clearly $B$ is a unital essential extension of $C\left(S^{1}\right)$ by $\mathcal{K}$. Therefore $B \cong \mathcal{T}_{0}$. Suppose that $\gamma(n)=l_{1} n$ for $n \in \mathbf{Z}_{+}$. From the case that $r=0$, we know that there exists a unital homomorphism $\tilde{\phi}_{j}^{\prime}: \mathcal{T}_{0} \rightarrow B$ such that $\tilde{\phi}_{j}^{\prime}$ induces a map from $V_{*}\left(\mathcal{T}_{0}\right)$ into $V_{*}(B)$ which has multiplicity $l_{1}$ on $I\left(F_{0}\right)$. Finally, we define $\tilde{\phi}_{j}=j \circ \tilde{\phi}_{j}^{\prime}$, where $j: B \rightarrow g\left(e_{11}\right) M_{m}\left(\mathcal{T}_{r}\right) g\left(e_{11}\right)$ is the embedding. It is easily checked that this $\tilde{\phi}_{j}$ meets the requirements.

Now we consider the case that $\gamma$ is trivial on $I\left(F_{k_{j}}\right)$. This is always the case if $k_{j}>0$. In fact, in this case, the map $\delta_{1}$ is injective, so $\alpha_{3}=0$. So $\alpha$ is not injective, by part (f) of $2.4, \alpha$ also maps $I\left(F_{k_{j}}\right)$ into zero.

We define $\tilde{\phi}_{j}=\lambda \circ \pi_{1}$, where $\lambda$ is a homomorphism from $C\left(S^{1}\right)$ into $g\left(e_{11}\right) M_{m}\left(\mathcal{T}_{r}\right) g\left(e_{11}\right)$ by sending $z$ into $W$. Clearly, $\tilde{\phi}_{j}$ meets the requirements.

Now we consider the case that $\alpha\left(\left[1-S_{k_{j}} S_{k_{j}}^{*}\right]\right) \neq 0$. Note that in this case it is necessary that $k_{j}>0$. Since $1-S_{k_{j}} S_{k_{j}}^{*}$ is the sum of $k_{j}$ mutually orthogonal and equivalent projections, say $q_{1}, \cdots, q_{k_{j}}$, there are $k_{j}$ mutually orthogonal and equivalent projections $a_{1}, \cdots, a_{k_{j}}$ such that $\alpha\left(\left[q_{i}\right]\right)=\left[a_{i}\right]=$ $\left[a_{1}\right]$ in $V\left(M_{m}\left(\mathcal{T}_{r}\right)\right)$ and

$$
\sum_{i=1}^{k_{j}} a_{i}=g\left(e_{11}\right)-W W^{*} .
$$

We now define $\tilde{\phi}_{j}$ from $e_{11} M_{k_{j}}\left(\mathcal{T}_{k_{j}}\right) e_{11} \cong \mathcal{T}_{k_{j}}$ to $g\left(e_{11}\right) M_{m}\left(\mathcal{T}_{r}\right) g\left(e_{11}\right)$ by sending $S_{k_{j}}$ to $W$ and by sending $\left(1-S_{k_{j}} S_{k_{j}}^{*}\right) I\left(\mathcal{T}_{k_{j}}\right)\left(1-S_{k_{j}} S_{k_{j}}^{*}\right)$ into $\left(g\left(e_{11}\right)-\right.$ $\left.W W^{*}\right) M_{m}(\mathcal{K})\left(g\left(e_{11}\right)-W W^{*}\right)$ By 2.1 , this is in fact a homomorphism. From our construction, it is clear that such defined $\tilde{\phi}_{j}$ induces a map from $V_{*}\left(e_{11} M_{m}\left(\mathcal{T}_{k_{j}}\right) e_{11}\right)$ into $V_{*}\left(M_{m}\left(\mathcal{T}_{r}\right)\right)$ which agrees with the restriction of $\alpha$.

Remark 3.2. We would like to remark that the condition for $\psi$ in the Theorem 3.1 is necessary. If $\phi: M_{m}\left(\mathcal{T}_{0}\right) \rightarrow M_{m^{\prime}}\left(\mathcal{T}_{k}\right)$ is a homomorphism and $\operatorname{ker} \phi \cap I\left(M_{m}\left(\mathcal{T}_{0}\right)\right)=0$, then $\operatorname{ker} \phi=0$, since $I\left(M_{m}\left(\mathcal{T}_{0}\right)\right)$ is an essential ideal of $M_{m}\left(\mathcal{T}_{0}\right)$. So $\phi$ is injective. To see that the induced map from $M_{m}\left(C\left(S^{1}\right)\right)$ into $M_{m^{\prime}}\left(S^{1}\right)$ is injective, we let $u$ be the unitary in $e_{11}\left(\mathcal{T}_{0}\right) e_{11}$ such that $\pi(u)=z$, where $z$ is the function in $e_{11} C\left(S^{1}\right) e_{11} \cong C\left(S^{1}\right)$ which is identity on $S^{1}$ and $\pi: M_{m}\left(\mathcal{T}_{0}\right) \rightarrow M_{m}\left(C\left(S^{1}\right)\right)$ is the quotient map. Since $\phi$ is injective, $s p(\phi(u))=S^{1}$. We want to show that, in fact, $s p\left(\pi_{1}(\phi(u))\right)=S^{1}$, where $\pi_{1}: M_{m^{\prime}}\left(\mathcal{T}_{k}\right) \rightarrow M_{m^{\prime}}\left(C\left(S^{1}\right)\right)$ is the quotient map. Suppose that 
$s p(\phi(u))=F$. If $F \neq S^{1}$, then, since $F$ is compact, $S^{1} \backslash F$ contains an arc, say $C$. Suppose that $C_{0} \in C$ is a sub-arc of $C$. Let $f \in C\left(S^{1}\right)$ such that $f(\xi)=1$, if $\xi \in C_{0}, f(\xi)=0$, if $\xi \in F$ and $f$ is linear otherwise. Let $a=\phi(u) \phi(f(u))$. Then $C_{0} \subset s p(a)$. However, $a \in I\left(M_{m^{\prime}}\left(\mathcal{T}_{k}\right)\right)(\cong \mathcal{K})$. So $s p(a)$ can have only countably many points. A contradiction. Therefore, $S^{1} \backslash F$ does not contain any arc, which implies that $F=S^{1}$.

3.3. The class of $A \mathcal{T}$-algebras of real rank zero will be denoted by $\mathbb{T}$. The subclass $\mathbb{T}_{1}$ consists of those $C^{*}$-algebras that there are no $M_{m}\left(\mathcal{T}_{0}\right)$ appeared in any summand in the direct limit system. We will call the algebras in these class $\mathbb{T}$-algebras and $\mathbb{T}_{1}$-algebras, respectively.

If $E$ is a unital $A \mathcal{T}$-algebra then $V(E)=\lim \left(V\left(E_{n}\right), \phi_{n, n+1}^{*}\right)$ is a direct limit of finite direct sums of $V\left(E_{n}\right)$. The homomorphism $\phi_{n, n+1}^{*}$ maps the subsemigroup $I\left(V\left(E_{n}\right)\right)$ into $I\left(V\left(E_{n+1}\right)\right)$. Let $I(V(E))$ be the subsemigroup $\lim I\left(V\left(E_{n}\right)\right)$. If we assume that $E$ has real rank zero, then $Q(V(E))$ is a positive cone of an unperforated ordered group with Riesz decomposition property. This follows from the fact that $Q(E)$ is an $A \mathbb{T}$-algebra with real rank zero and Theorem 8.1 of [Ell2].

Let $V=\lim _{\rightarrow}\left(V_{n}, \alpha^{n, n+1}\right)$ be a direct limit of finite direct sums of $F_{k(n)}^{\prime} s$. $(G(V), V)$ is an ordered group. By 2.10 , there are maps $\alpha_{5}^{n, n+1}: Q\left(V_{n}\right) \rightarrow$ $Q\left(V_{n+1}\right)$ induced by $\alpha$. Let $V_{*}=\lim _{\rightarrow}\left(V_{n *}, \alpha^{n, n+1}\right)$ be a direct limit of finite direct sums of $F_{k(n) *}^{\prime} s$. By 2.10, there are also maps $\alpha_{5}^{n, n+1}$ from $Q\left(V_{n *}\right)$ into $Q\left(V_{n+1 *}\right)$. We will identify $V$ with the semigroup $\lim _{\rightarrow}\left(V_{n}, \alpha^{n, n+1}\right)$ of $V_{*}$.

Lemma. Let $E=\lim \left(E_{n}, \phi_{n, n+1}\right)$ be a direct limit of finite direct sums of $\mathcal{T}$-algebras of real rank zero. Then, for any $k$ and any summand $E_{k}^{i} \cong$ $M_{m}\left(\mathcal{T}_{0}\right)$ of $E_{k}$, there exists an integer $N(k)>0$ such that every partial homomorphism $\phi_{k, n}^{i, j}$ is either non-injective or the multiplicity of $\left(\psi_{k, n}^{i, j}\right)_{* 0}$ is greater than 1 for all $n \geq N$, where $\psi_{k, n}$ is the homomorphism from $Q\left(E_{k}^{i}\right)$ into $Q\left(E_{n}^{j}\right)$ induced by $\phi_{k, n}^{i, j}$.

Proof. Suppose that the lemma is false. We may assume, by taking a subsequence, that $E^{\prime} \cong M_{m}^{\prime}\left(\mathcal{T}_{0}\right)$ is a summand of $E_{1}$ and $\phi_{1, n}^{1, j_{n}}$ is injective and $\left(\psi_{1, n}^{1, j_{n}}\right)_{* 0}$ has multiplicity 1 for $j_{n}$ and for each $n$. Let $\psi_{n, n+1}$ be the homomorphism from $Q\left(E_{n}\right)$ into $Q\left(E_{n+1}\right)$ induced by $\phi_{n, n+1}$. Let $p=\psi_{1, \infty}\left(z^{*} z\right)$, where $z$ is the canonical unitary in $e_{11} M_{m}\left(C\left(S^{1}\right)\right) e_{11}$. Then $p Q(E) p$ is an $A \mathbb{T}$-algebra of real rank zero. Let $h=\left(z+z^{*}\right) / 2$ and $x=\psi_{1, \infty}(h)$. Since $p Q(E) p$ has real rank zero, for any $1 / 2 \varepsilon>0$, there is a selfadjoint element $y \in p Q(E) p$ with finite spectrum such that

$$
\|x-y\|<\varepsilon / 4 .
$$


Suppose that $y=\sum_{l=1}^{L} \gamma_{l} e_{l}$, where $\gamma_{l}$ are real numbers and $e_{l}$ are mutually orthogonal projections in $p Q(E) p$. Without loss of generality, by taking a large $n$, we may assume that $e_{l} \in \psi_{n, \infty}\left(\psi_{1, n}\left(z^{*} z\right) Q\left(E_{n}\right) \psi_{1, n}\left(z^{*} z\right)\right)$. We may also assume, by taking large $n$, without loss of generality, that

$$
\left\|\psi_{1, n}(h)-g\right\|<\varepsilon / 2,
$$

where $g$ is selfadjoint element in $\psi_{1, n}\left(z^{*} z\right) Q\left(E_{n}\right) \psi_{1, n}\left(z^{*} z\right)$ and $\psi_{n, \infty}(g)=y$. Let $f_{l}$ be selfadjoint element in $\psi_{1, n}\left(z^{*} z\right) Q\left(E_{n}\right) \psi_{1, n}\left(z^{*} z\right)$ such that $\psi_{n, \infty}\left(f_{l}\right)=e_{l}$. Since

$$
\left\|\psi_{n, k}\left(f_{l}\right)^{2}-\psi_{n, k}\left(f_{l}\right)\right\| \rightarrow 0
$$

as $k \rightarrow \infty$, by functional calculus, we may further assume that $g$ has finite spectrum.

Now $e=\psi_{1, n}^{1, j_{n}}\left(z^{*} z\right)$ commutes with both $\psi_{1, n}(h)$ and $g$. Since $\psi_{1, n}^{1, n}$ is injective and $\left(\psi_{1, j_{n}}^{1, n}\right)_{* 0}$ has multiplicity $1, e Q\left(E_{n}\right) e \cong C\left(S^{1}\right)$. We have $\psi_{1, n}^{1, j_{n}}(h)(=$ $\left.e \psi_{1, n}(h)\right)$, ege $\in e Q\left(E_{n}\right) e \cong C\left(S^{1}\right)$, ege has finite spectrum and

$$
\left\|\psi_{1, n}^{1, j_{n}}(h)-e g e\right\|<\varepsilon / 2 .
$$

Since $\operatorname{sp}\left(\psi_{1, n}^{1, j_{n}}(h)\right)=[-1,1]$, and ege has at most 1 point in its spectrum, the above inequality could not hold.

Proposition 3.4. Let $E=\lim _{\rightarrow}\left(E_{n}, \phi_{n, n+1}\right)$ be an $A \mathcal{T}$-algebra of real rank zero. Denote by $\psi_{n, n+1}$ the *-homomorphism from $Q\left(E_{n}\right)$ to $Q\left(E_{n+1}\right)$ induced by $\phi_{n, n+1}$. Then there exists a subsequence $\{n(k)\}$ of integers such that each partial homomorphism of $\left(\phi_{n(k), n(k+1)}\right)_{* 0}$ is either zero when restricted on any summand $I\left(F_{0}\right)$, or the corresponding partial homomorphism of $\left(\psi_{n(k), n(k+1)}\right)_{* 0}$ has multiplicity $>2^{k+1}$.

Proof. This is an immediate consequence of the proof of Lemma 3.3.

Definition 3.5. Let $V=\lim _{\rightarrow}\left(V_{n}, \alpha^{n, n+1}\right)$ be a direct limit of finite direct sums of $F_{n(k) *}$ 's. We say $V$ satisfies generalized Riesz decomposition property if $(G(Q(V)), Q(V))$ is an unperforated ordered group with Riesz decomposition property and there exists a subsequence $\{n(k)\}$ of integers such that every partial homomorphism $\alpha_{n(k), n(k+1)}^{\prime}$ restricted on any summand $I\left(F_{0}\right)$ is either zero or the corresponding partial homomorphism $\alpha_{5}^{n(k), n(k+1)^{\prime}}$ has multiplicity $>2^{k+1}$, where $\alpha_{5}^{n(k), n(k+1)}$ is the homomorphism from $Q\left(V_{n(k)}\right)$ into $Q\left(V_{n(k+1)}\right)$ induced by $\alpha_{n(k), n(k+1)}$.

So we have the following.

Proposition 3.6. If $E$ is an $A \mathcal{T}$-algebra of real rank zero, then $V(E)_{*}$ has the generalized Riesz decomposition property.

Proof. This follows immediately from 3.4 and 3.3 . 


\section{Proposition 3.7.}

(A) Let $V=\lim \left(V_{n}, \alpha^{n, n+1}\right)$, where $V_{n}=\oplus_{i=1}^{m(n)} F_{(n, i)}$ and $(G(Q(V)), Q(V))$ has the Riesz decomposition property. Then, for a fixed order unit $q(e) \in Q(V)$, where $e \in V$, there exists a unital $\mathbb{T}_{1}$-algebra $E=$ $\lim _{\rightarrow}\left(E_{n}, \phi_{n, n+1}\right)$ with

$$
V(E)=V,[1]=e .
$$

(B) Let $V_{*}=\lim _{\rightarrow}\left(V_{n *}, \alpha^{n, n+1}\right)$, where $V_{n *}=\oplus_{i=1}^{m(n)} F_{(n, i) *}$, has the generalized Riesz decomposition property. Then, for a fixed order unit $q(e) \in Q(V)$, where $e \in V$, there exists a unital $\mathbb{T}$-algebra, E such that

$$
V_{*}(E)=V_{*},[1]=e .
$$

Proof. We first find an AT-algebra $Q(E)$ of real rank zero which will gives

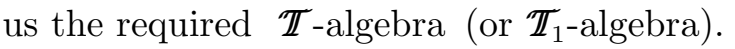

For case (A), let $j_{n}: V_{n} \rightarrow G\left(V_{n}\right)$ and $j: V \rightarrow G(V)$ be the natural homomorphism from $V_{n}$ and $V$ into their Grothendieck groups. It is easy to see that

$$
\operatorname{ker} j=\lim _{\rightarrow}\left(\operatorname{ker} j_{n}, \alpha^{n, n+1}\right) \text {. }
$$

We will denote $k\left(V_{n}\right)_{+}=\operatorname{ker} j_{n}$ and $k(V)_{+}=\operatorname{ker} j$. We write $\left(Q\left(V_{n}\right) \oplus\right.$ $\left.k\left(V_{n}\right)_{+}, \Gamma_{n}\right)$ for the direct sum of summands $\mathbf{Z}_{+} \oplus k_{i} \mathbf{Z}_{+}$with order in each such summand determinded by the strict order in the first component. Let $\alpha_{5}^{n, n+1}: Q\left(V_{n}\right) \rightarrow Q\left(V_{n+1}\right)$ be the quotient map induced by $\alpha^{n, n+1}$ and let $\alpha_{2}^{n, n+1}: k\left(V_{n}\right)_{+} \rightarrow k\left(V_{n+1}\right)_{+}$be the restriction of $\alpha^{n, n+1}$. One can extend $\alpha_{5}^{n, n+1}$ to group homomorphisms from $G\left(Q\left(V_{n}\right)\right)$ into $G\left(Q\left(V_{n+1}\right)\right)$ and from $k\left(V_{n}\right)_{+}=G\left(k\left(V_{n}\right)\right)_{+}$into $k\left(V_{n+1}\right)_{+}=G\left(k\left(V_{n+1}\right)\right)_{+}$, respectively. We will use $\alpha_{5}^{n, n+1}$ for the first extension, $\alpha_{2}^{n, n+1}$ for the second extension and let $\eta^{n, n+1}=\alpha_{5}^{n, n+1} \oplus \alpha_{2}^{n, n+1}$. Let $k\left(V_{n}\right)=G\left(k\left(V_{n}\right)_{+}\right)$and $k(V)=G\left(k(V)_{+}\right)$We then denote

$$
(G(Q(V)) \oplus k(V), \Gamma)=\lim _{\rightarrow}\left(G\left(Q\left(V_{n}\right)\right) \oplus k\left(V_{n}\right), \Gamma_{n}, \eta^{n, n+1}\right) .
$$

For case (B), let

$$
Q_{*}\left(V_{n}\right)=\left\{(\pi(x), y):(x, y) \in V_{n *}\right\},
$$

where $\pi: V_{n} \rightarrow Q\left(V_{n}\right)$ is the quotient map. Define $\eta^{n, n+1}(\pi(x), y)=$ $\left(\pi\left(\alpha^{n, n+1}(x)\right), \alpha_{n, n+1}(y)\right)(x, y) \in V_{n *}$. Let

$$
k(V)_{+}=\lim _{\rightarrow}\left(k\left(V_{n}\right)_{+}, \alpha^{n, n+1}\right) .
$$


We also note that

$$
Q(V)=\lim _{\rightarrow}\left(Q\left(V_{n}\right), \alpha^{n, n+1}\right) .
$$

Then $k(V)=G\left(k(V)_{+}\right)=\lim _{\rightarrow} G\left(k\left(V_{n}\right)_{+}\right)$and $G(Q(V))=\lim _{\rightarrow} G\left(Q\left(V_{n}\right)\right)$. Define

$$
\Gamma=\lim _{\rightarrow}\left(Q_{*}\left(V_{n}\right), \alpha_{5}^{n, n+1} \oplus \alpha_{2}^{n, n+1}\right) .
$$

For both case (A) and (B), since $Q(G(V))$ has Riesz decomposition property, the asymptotic condition (ii) in Theorem 7.2 of [Ell2], implies that $(Q(G(V)) \oplus k(V), \Gamma)$ (for both case (A) and (B)) has the asymptotic property (ii) in Theorem 8.1 of [Ell2]. Therefore, by Theorem 8.1 of [Ell2], $(G(Q(V)) \oplus k(V), \Gamma)$ (in both cases) has Riesz decomposition property. We also assume that $e_{n} \in V_{n}$ such that $\alpha^{n, n+1}\left(e_{n}\right)=e_{n+1}$ and the image of $e_{n}$ is $e \in V$.

Now by Lemma 7.2 of [E112], by passing to a subsequence if necessary, we may assume the following.

(1): For every $n \in \mathbf{N}$, every summand $\mathbf{Z}$ of $Q\left(V_{n}\right)$, and every summand $\mathbf{Z}$ of $Q\left(V_{n+1}\right)$, the partial homomorphism $\alpha_{5}^{n, n+1^{\prime}}: \mathbf{Z} \rightarrow \mathbf{Z}$ has multiplicity $m_{0} \geq 2^{n+1}$ or the corresponding partial homomorphism $\alpha_{2}^{n, n+1^{\prime}}$ is zero.

(2): if $\alpha_{2}^{n, n+1^{\prime}}=0$ but $\alpha_{3}^{n, n+1^{\prime}} \neq 0$ the partial homomorphism $\alpha_{5}^{n, n+1}$ has multiplicity $m_{0}>2^{n+1}$.

To see (2), we note that if the corresponding summand is $F_{r *}$ with $r>0$, $\alpha_{3}^{n, n+1^{\prime}} \neq 0$, implies that $\alpha_{2}^{n, n+1} \neq 0$, so $m_{0}>2^{n+1}$. If $r=0$, then we apply the second condition of the generalized Riesz decomposition property.

Let

$$
A_{n}=\oplus_{i=1}^{m(n)} M_{k(n, i)}\left(C\left(S^{1}\right)\right),
$$

where each summand $M_{k(n, i)}\left(C\left(S^{1}\right)\right)$ corresponds a summand of $Q\left(G\left(V_{n}\right)\right)$. Here $m(n)$ is determines by $Q\left(G\left(V_{n}\right)\right)$. For $k(n, i)$, we let $k(1, i)=1$ and $k(n, i)$ defined inductively by homomorphisms $\alpha^{n, n+1}$ as follows.

Suppose that there are $m(n+1)$ copies of $\mathbf{Z}$ in $Q\left(G\left(V_{n}\right)\right)$ and

$$
q\left(e_{n+1}\right)=(k(n+1,1), k(n+1,2), \ldots, k(n+1, m(n+1))),
$$

where $q: G\left(V_{n}\right) \rightarrow Q\left(G\left(V_{n}\right)\right)$ is the quotient map. Since $q(e)$ is an order unit, without loss of generality, by passing a subsequence if necessary, we may assume that $k(n+1, i)$ is positive for each $i$. Set

$$
A_{n+1}=\oplus_{i=1}^{m(n+1)} M_{k(n+1, i)}\left(C\left(S^{1}\right)\right) .
$$

Note that

$$
K_{0}\left(A_{n}\right)=Q\left(G\left(V_{n}\right)\right) \quad \text { and } \quad K_{1}\left(A_{n}\right)=k(V) .
$$


We define the partial homomorphism

$$
\psi_{n, n+1}^{\prime}: M_{k(n, i)}\left(C\left(S^{1}\right)\right) \rightarrow M_{k(n+1, j)}\left(C\left(S^{1}\right)\right)
$$

as follows. If $\alpha_{2}^{n, n+1^{\prime}}=0$ and $\alpha_{3}^{n, n+1^{\prime}}=0$, define

$$
\psi_{n, n+1}^{\prime}(f)=\operatorname{diag}(f(1), \cdots f(1), 0, \cdots, 0) \in M_{k(n+1, j)}\left(C\left(S^{1}\right)\right)
$$

(there are $m_{0}$ many $f(1)$ ). If $\alpha_{2}^{n, n+1^{\prime}}=0$ and $\alpha_{3}^{n, n+1} \neq 0$, (in this case, $\left.m_{0}>2^{n+1}\right)$, define

$$
\psi_{n, n+1}^{\prime}(f)=\operatorname{diag}(f(z), f(1), \cdots, f(1), 0, \cdots, 0) \in M_{k(n+1, j)}\left(C\left(S^{1}\right)\right)
$$

(there are $m_{0}-1$ many $f(1)$ ).

If $\alpha_{2}^{n, n+1^{\prime}}$ maps a generator of $i$ th summand of $k\left(V_{n}\right)$ to $m_{1}$ times a generator of $j$ th summand of $k\left(V_{n+1}\right)$ and $m_{1} \neq 0$, then set

$$
\begin{aligned}
& \psi_{(n, n+1)}^{\prime}(f) \\
& =\operatorname{diag}\left(f\left(z^{m_{1}-1}\right), f(\omega), f\left(\omega^{2}\right), f\left(\omega^{3}\right), \cdots, f\left(\omega^{m_{0}}\right), 0, \cdots, 0\right) \in M_{m^{\prime}}\left(C\left(S^{1}\right)\right),
\end{aligned}
$$

where $\omega=\exp \left(2 \pi i /\left(m_{0}-2\right)\right)$. The construction of $\psi_{n, n+1}$ ensures that it induces the same map $K_{0}\left(A_{n}\right) \oplus K_{1}\left(A_{n}\right) \rightarrow K_{0}\left(A_{n+1}\right) \oplus K_{1}\left(A_{n+1}\right)$ as $\left(\alpha_{5}^{n, n+1} \oplus\right.$ $\left.\alpha_{2}^{n, n+1}\right)$. Let

$$
A=\lim _{\rightarrow}\left(A_{n}, \psi_{n, n+1}\right) .
$$

Then $A$ is of real rank zero. This is certainly known. We sketch the proof as follows. Let $h$ be a selfadjoint function in a summand of $A_{n}$. We want to show that, for any $\varepsilon>0$, there are $m>n$ and selfadjoint element $h^{\prime}$ in $A_{m}$ with finite spectrum such that $\left\|\psi_{n, m}^{\prime}(h)-h^{\prime}\right\|<\varepsilon$ for each partial map $\psi_{n, m}^{\prime}$ from $A_{n}$ into $A$. Using an $\varepsilon / 16$-cover, dividing the spectrum into finitely many disjoint pieces, write $h$ as a direct sum of selfadjoint elements and then shift the spectrum, without loss of generality, we may assume that $s p(h)$ is in $[0,\|h\|]$ and it is $\varepsilon / 8$-dense in $[0,\|h\|]$. Take a large $m>n$,

$$
\psi_{n, m}^{\prime}(h)=\operatorname{diag}\left(h\left(z^{m_{1}^{\prime}-1}\right), h(\omega), h\left(\omega^{2}\right), \ldots, g\left(\omega^{m_{0}}\right), 0, \ldots, 0\right) .
$$

Note that $\left\{h(\omega), h\left(\omega^{2}\right), \ldots, h\left(\omega^{m_{0}}\right)\right\}$ is $\varepsilon / 4$ dense in $[0,\|h\|]$, if $m$ is large enough. One then applies (i) of Lemma 8 in $[\mathbf{L R}]$ to obtain the assertion.

Let $M_{k(n, i)}\left(C\left(S^{1}\right)\right)$ correspond to a summand $F_{l(n, i)}$. We will lift $M_{k(n, i)}$ to $M_{k(n, i)}\left(\mathcal{T}_{l(n, i)}\right)$. Define $E_{n}^{\prime}=\oplus_{i=1}^{m(n)} M_{k(n, i)}\left(\mathcal{T}_{l(n, i)}\right)$. Then $V\left(E_{n}^{\prime}\right) \oplus V_{n}^{\prime \prime}=V_{n}$ for case (A) and $V_{*}\left(E_{n}^{\prime}\right) \oplus V_{n *}^{\prime \prime}=V_{n *}$ for case (B), where $\alpha^{n, n+1}\left(V_{n}^{\prime \prime}\right) \in I\left(V_{n+1}\right)$ in both cases. There are, for each $n$, a finite direct sum of $\mathcal{T}$-algebras $E_{n}^{\prime \prime}$ such that $V_{n}^{\prime \prime}=V\left(E_{n}^{\prime \prime}\right)$ (for case $\left.(\mathrm{A})\right)$ or $V_{n *}^{\prime \prime}=V_{*}\left(E_{n}^{\prime \prime}\right)$. Let $E_{n}=E_{n}^{\prime} \oplus E_{n}^{\prime \prime}$. We have 
$V\left(E_{n}\right)=V_{n}, V_{*}\left(E_{n}\right)=V_{n *}$ for case $(\mathrm{B}), V\left(I\left(E_{n}\right)\right)=I\left(V_{n}\right), K_{0}\left(Q\left(E_{n}\right)\right)=$ $G\left(Q\left(\left(V_{n}\right)\right)\right)$ and $K_{1}\left(Q\left(E_{n}\right)\right)=G\left(k\left(V_{n}\right)\right)$. Remember that $\alpha^{n, n+1}$ induces $\alpha_{5}^{n, n+1} \oplus \alpha_{2}^{n, n+1}$.

We now use the second condition in the generalized Riesz decomposition property to apply 3.1. By 3.1, we obtain the following commutative diagram:

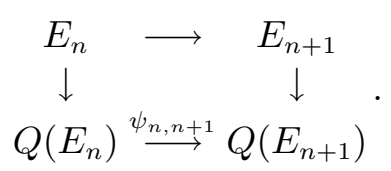

Then the direct limit $E=\lim _{\rightarrow} E_{n}$ has $V(E)=V,[1]=e$, or $V_{*}(E)=V_{*}$ for case (B). Note $A_{n}=Q\left(E_{n}\right)$. We have

$$
Q(E)=\lim _{\rightarrow}\left(A_{n}, \psi_{n, n+1}\right) .
$$

Since $A$ has real rank zero, it follows from 2.3 of [Zh1] and 2.11 of [Zh2] that $E$ has real rank zero.

Lemma 3.8. Every AF-algebra is an $\mathbb{T}_{1}$-algebra. Furthermore, we can choose $E_{n}$ in the direct limit such that each summand is a matrix algebra over $\mathcal{T}_{1}$.

Proof. Let $A$ be a unital $A F$-algebra and $G$ be its dimension group. Let $G=\lim \left(G_{n}, \eta_{n}\right)$, where each $G_{n}$ is a direct sum of finitely many copies of $\mathbf{Z}$. Let $V_{n}$ be the corresponding sum of $\mathbf{Z}_{+}$. Let $V_{n}^{(i)}$ be a summand $\left(V_{n}^{(i)}=\mathbf{Z}_{+}\right)$. Let $R_{n}^{(i)}=\mathbf{Z}_{+} \sqcup V_{n}^{(i)}$. Define $x+y=y$, if $x \in \mathbf{Z}_{+}, y \neq 0$ and $y \in V_{n}^{(i)}$. Let $V_{n}^{\prime}=\oplus R_{n}^{(i)}$.

Now define a map $\eta_{n}^{\prime}: V_{n}^{\prime} \rightarrow V_{n+1}^{\prime}$ by defining $\left.\eta_{n}^{\prime}\right|_{R_{n}^{(i)}}(x)=0$, if $x \in \mathbf{Z}_{+}$and $\left.\eta_{n}^{\prime}\right|_{R_{n}^{(i)}}(y)=\left.\eta_{n}\right|_{V_{n}^{(i)}}(y)$. Then $V=\lim _{\rightarrow}\left(V_{n}^{\prime}, \eta_{n}^{\prime}\right)=\lim _{\rightarrow}\left(V_{n}, \eta_{n}\right)$. It follows from 3.7 that there is a $\mathbb{T}_{1}$-algebra $E$ such that $V(E)=V$. Since $I(V)=\{0\}$, $I(E)=0$. Therefore, $E \cong Q(E)$. This implies that $K_{0}(Q(E))=G$ as ordered group and $K_{1}(Q(E))=\{0\}$. Since $Q(E)$ is an $A \mathbb{T}$-algebra, by Theorem 7.1 of $[\mathbf{E l l 2}], E \cong Q(E) \cong A$.

Lemma 3.9. Every AT-algebra of real zero is in $\mathbb{T}$.

Proof. Let $A=\lim _{\rightarrow}\left(A_{n}, \rho_{n, n+1}\right)$ be an $A \mathbb{T}$-algebra, where

$$
A_{n}=\oplus_{j=1}^{L_{n}} M_{k_{j}}\left(C\left(S^{1}\right)\right) .
$$

Set

$$
E_{n}=\oplus_{j=1}^{L_{n}} M_{k_{j}}\left(\mathcal{T}_{0}\right)
$$


Let $\pi_{n}: E_{n} \rightarrow A_{n}$ be the quotient map. There is a unital monomorphism $j_{n}: A_{n} \rightarrow E_{n}$ such that $j_{n} \circ \pi_{n}$ is the identity map on $A_{n}$. We define $\phi_{n, n+1}=j_{n+1} \circ \rho_{n, n+1} \circ \pi_{n}$. So $E=\lim _{\rightarrow}\left(E_{n}, \phi_{n, n+1}\right)$ is an $A \mathcal{T}$-algebra. Clearly $I(E)=0$ and $E \cong A$. So $E$ has real rank zero. Therefore $E \in \mathbb{T}$.

3.10. Our invariants for $\mathbb{T}$ are $V_{*}(-)$. However, we would like briefly describe the $K$-theory of $\mathbb{T}$. If $E \in \mathbb{T}$, then $K_{0}(E)=G(V(E))$ and $K_{1}(E)=$ $G(\operatorname{ker} d)$. The map from ker $d$ into $G($ ker $d)$ is injective. In particular, if $E \in \mathbb{T}_{1}, K_{1}(E)=0$. In general, one may write $K_{0}(E)=G_{0} \oplus G_{1}$, where $G_{1}$ can be any countable torsion group that every element in it has finite order. The order of $K_{0}(E)$ is determined by $G_{0}$, where $G_{0} / K_{0}(I(E))$ is any countable abelian unperforated ordered group with the Riesz decomposition property. Note that $I(E)$ is a stable $A F$-algebra.

\section{Uniqueness.}

In this section, we will prove a so called uniqueness theorem for homomorphisms from one matrix algebra over $\mathcal{T}$-algebra to another.

Lemma 4.1 ([Ln5, 2.1]). Every derivable automorphism on a unital separable $C^{*}$-algebra $A$ is approximately inner.

4.2. Denote the function $f_{1}\left(\left(e^{i \theta}, e^{i \gamma}\right)\right)=e^{i \theta}$ and $f_{2}\left(\left(e^{i \theta}, e^{i \gamma}\right)\right)=e^{i \gamma}$ in $C\left(S^{1} \times S^{1}\right)$, respectively. Then $C\left(S^{1} \times S^{1}\right)$ is generated by $f_{1}$ and $f_{2}$. Let $X$ be a compact subset of $S^{1} \times S^{1}$. Then there is a homomorphism $\phi: C\left(S^{1} \times S^{1}\right) \rightarrow C(X)$. We will also use the notation $f_{1}$ and $f_{2}$ for $\phi\left(f_{1}\right)$ and $\phi\left(f_{2}\right)$, if there is no confusion. Note that $f_{1}$ and $f_{2}$ also generate $C(X)$. In the rest of this section, $f_{1}$ and $f_{2}$ will always mean these functions.

Lemma. Let $X$ be a compact subset of $S^{1} \times S^{1}$ and let $\tau: C(X) \rightarrow$ $B(H) / \mathcal{K}$ be a unital trivial essential extension of $C(X)$ by $\mathcal{K}$. Suppose that $s p\left(\tau\left(f_{2}\right)\right)=F\left(\subset S^{1}\right)$. Then there exists a norm continuous path of unitaries $\left\{w_{t}\right\}$ in $B(H) / \mathcal{K}$ such that

$$
w_{0}=\tau\left(f_{1}\right), \quad\left[w_{t}, \tau\left(f_{2}\right)\right]=0,
$$

and the $C^{*}$-subalgebra $C^{*}\left(w_{1}, \tau\left(f_{2}\right)\right)$ is isomorphic to $C\left(S^{1} \times F\right)$.

Proof. Suppose that $\lambda \in X$. Then there exists a nonzero projection $q \in$ $B(H) / \mathcal{K}$ such that

$$
\tau(f) q=q \tau(f)=f(\lambda) q
$$

for all $f \in C(X)$ (see [BDF2]). By taking a subprojection of $q$ if necessary, we may assume that the map $\tau^{\prime}(f)=\tau(f)(1-q)$ is still injective. Note 
that $f_{2}(\lambda)=\exp (i a)$ for some nonnegative number $a \leq 2 \pi$. Let $Q$ be a projection in $B(H)$ such that $\pi(Q)=q$, where $\pi: B(H) \rightarrow B(H) / \mathcal{K}$ is the quotient map. Then $\tau^{\prime}$ gives a unital trivial essential extension of $C(X)$ by $(1-Q) \mathcal{K}(1-Q)$.

By the BDF theory (see [BDF1]), there is an abelian AF-algebra $B \subset$ $(1-q)(B(H) / \mathcal{K})(1-q)$ such that im $\tau^{\prime} \subset B$. Therefore there is $0 \leq h_{1} \leq$ $(2 \pi)(1-q)$ in $B$ such that $\tau^{\prime}\left(f_{1}\right)=\exp \left(i h_{1}\right)(1-q)$. Thus $\left[h_{1}, \tau^{\prime}\left(f_{2}\right)\right]=0$. Note also that

$h_{1} \tau\left(f_{2}\right)=h_{1}(1-q) \tau\left(f_{2}\right)=h_{1} \tau^{\prime}\left(f_{2}\right)=\tau^{\prime}\left(f_{2}\right) h_{1}=\tau\left(f_{2}\right)(1-q) h_{1}=\tau\left(f_{2}\right) h_{1}$.

Let $\tau_{0}: C\left(S^{1} \times F\right) \rightarrow q(B(H) / \mathcal{K}) q$ be a unital trivial essential extension of $C\left(S^{1} \times F\right)$ by $Q \mathcal{K} Q$. Again, there is $0 \leq h_{2} \leq(2 \pi) q$ in $B(H) / \mathcal{K}$ such that

$$
\exp \left(i h_{2}\right) q=\tau_{0}\left(f_{1}\right) \quad \text { and } \quad\left[h_{2}, \tau_{0}\left(f_{2}\right)\right]=0 .
$$

Set $h_{3}=h_{1}+h_{2}$. Then $0 \leq h_{3} \leq 2 \pi$ and

$$
\left[h_{3}, \tau^{\prime}\left(f_{2}\right)+\tau_{0}\left(f_{2}\right)\right]=0 .
$$

Note that $\operatorname{sp}\left(\tau^{\prime}\left(f_{2}\right)\right)=F$ and $\left[\tau^{\prime}(f)+\tau_{0}\left(f_{2}\right)\right]=\left[\tau\left(f_{2}\right)\right]=0$ in $K_{1}(B(H) / \mathcal{K})$. There is a unitary $W \in B(H) / \mathcal{K}$ such that

$$
W^{*}\left(\tau^{\prime}\left(f_{2}\right)+\tau_{0}\left(f_{2}\right)\right) W=\tau\left(f_{2}\right) .
$$

Set $h_{4}=W^{*} h_{3} W$. Then $\left[h_{4}, \tau\left(f_{2}\right)\right]=0$ and the $C^{*}$-subalgebra generated by $\exp \left(i h_{4}\right)\left(=W^{*}\left(\tau^{\prime}\left(f_{1}\right)+\tau_{0}\left(f_{1}\right)\right) W\right)$ and $\tau\left(f_{2}\right)$ is isomorphic to $C\left(S^{1} \times F\right)$. Now define $h_{1}^{\prime}=h_{1}+a q$ and

$$
w_{t}=\exp \left(i(1-t) h_{1}^{\prime}+t h_{4}\right) \quad t \in[0,1] .
$$

Note that

$$
w_{0}=\exp \left(i h_{1}^{\prime}\right)=\tau^{\prime}\left(f_{1}\right)+\exp (i a) q=\tau\left(f_{1}\right) \quad \text { and } \quad w_{1}=\exp \left(i h_{4}\right) .
$$

Since $\left[\tau\left(f_{2}\right), h_{1}^{\prime}\right]=\left[\tau\left(f_{2}\right), h_{4}\right]=0$,

$$
\left[w_{t}, \tau\left(f_{2}\right)\right]=0 \quad t \in[0,1]
$$

Lemma 4.3. Let $u$ and $v$ be two commuting unitaries in $B(H) / \mathcal{K}$ with $[u]=0$ in $K_{1}(B(H) / \mathcal{K})$ and $s p(v)=S^{1}$. Then there is a norm continuous path of unitaries $\left\{u_{t}\right\} \subset B(H) / \mathcal{K}$ such that

$$
u_{0}=u, u_{1}=1 \quad \text { and } \quad\left[u_{t}, v\right]=0
$$


for $0 \leq t \leq 1$.

Proof. Let $\phi: C(X) \rightarrow B(H) / \mathcal{K}$ be the monomorphism generated by $u$ and $v$ such that $\phi\left(f_{1}\right)=u$ and $\phi\left(f_{2}\right)=v$. We will assume that $s p(u)=S^{1}$. Otherwise, there is a selfadjoint element $h \in B(H) / \mathcal{K}$ such that $\exp (i h)=u$ and $[h, v]=0$. We can take $u_{t}=\exp (i(1-t) h)$.

We consider two cases.

(1) We assume that $C^{*}(u, v)=C\left(S^{1} \times S^{1}\right)$. Take a unital trivial essential extension $\tau_{0}: C\left(S^{1} \times S^{1}\right) \rightarrow B(H) / \mathcal{K}$. Since $\tau_{0}$ is trivial, as in the proof of Lemma 4.2 , there is $0 \leq h_{1} \leq 2 \pi$ in $B(H) / \mathcal{K}$ such that $\tau_{0}\left(f_{1}\right)=\exp \left(i h_{1}\right)$ and $\left[h_{1}, \tau_{0}\left(f_{2}\right)\right]=0$.

Define a unital extension $\tau_{1}$ of $C\left(S^{1} \times S^{1}\right)$ by defining $\tau_{1}\left(f_{1}\right)=1 \oplus \tau_{0}\left(f_{1}\right)$ and $\tau_{1}\left(f_{2}\right)=v \oplus \tau_{0}\left(f_{2}\right)$. Let $\left(\tau_{1}\right)_{*}$ and $\phi_{*}$ be the map from $K_{1}\left(C\left(S^{1} \times S^{1}\right)\right)$ into $K_{1}(B(H) / \mathcal{K})$ induced by $\tau_{1}$ and $\phi$. It is easy to check that $\left(\tau_{1}\right)_{*}=\phi_{*}$. By the BDF theory (see Theorem 6.6 of [BDF2]), there exists a unitary $W \in B(H) / \mathcal{K}$ such that

$$
W^{*}\left(\tau_{1}\right) W=\phi
$$

In particular

$$
W^{*} \tau_{1}\left(f_{1}\right) W=u \quad \text { and } \quad W^{*} \tau_{1}\left(f_{2}\right) W=v .
$$

Therefore, by setting $h_{1}^{\prime}=W^{*}\left(1 \oplus h_{1}\right) W$,

$$
u=\exp \left(i\left(h_{1}^{\prime}\right)\right) \quad \text { and } \quad\left[h_{1}^{\prime}, v\right]=0 .
$$

Now set

$$
w_{t}=\exp \left(i(1-t) h_{1}^{\prime}\right)
$$

This proves the case (1).

(2) General case, $C^{*}(u, v)=C(X)$, for some compact subset of $S^{1} \times S^{1}$. By the BDF theory, $\phi=\tau_{2} \oplus \tau_{0}$, where $\tau_{2}$ is unitarily equivalent to $\phi$ and $\tau_{0}: C(X) \rightarrow B(H) / \mathcal{K}$ is a unital essential trivial extension. Write

$$
\tau_{2}\left(f_{1}\right)=u^{\prime}, \tau_{2}\left(f_{2}\right)=v^{\prime}, \tau_{0}\left(f_{1}\right)=u^{\prime \prime} \quad \text { and } \quad \tau_{0}\left(f_{2}\right)=v^{\prime \prime} .
$$

So $u=u^{\prime} \oplus u^{\prime \prime}$ and $v=v^{\prime} \oplus v^{\prime \prime}$. Suppose that $\operatorname{sp}\left(\phi\left(f_{2}\right)\right)=S^{1}$, then $X \subset$ $S^{1} \times S^{1}$. By Lemma 4.2, there is a norm continuous path of unitaries $\left\{w_{t}\right\}$ in $B(H) / \mathcal{K}$ such that

$$
w_{0}=u^{\prime \prime},\left[w_{t}, v^{\prime \prime}\right]=0
$$

and $C^{*}\left(w_{1}, v^{\prime \prime}\right) \cong C\left(S^{1} \times S^{1}\right)$. Set $z_{t}=u^{\prime} \oplus w_{t}$. Then

$$
z_{0}=u,\left[z_{t}, v\right]=0 \quad \text { and } \quad C^{*}\left(z_{1}, v\right) \cong C\left(S^{1} \times S^{1}\right) .
$$


So we can then apply the case (1).

Theorem 4.4. Let

$$
0 \rightarrow \mathcal{K} \rightarrow E^{\prime} \rightarrow C\left(S^{1}\right) \rightarrow 0
$$

be a unital essential extension. Suppose that $\alpha: E^{\prime} \rightarrow E^{\prime}$ is an automorphism such that $\pi \circ \alpha=\pi$, where $\pi: E^{\prime} \rightarrow C\left(S^{1}\right)$ is the quotient map. Then $\alpha$ is approximately inner.

Proof. It is well known that there exists a unitary $W \in B(H)$ such that

$$
\alpha(a)=W^{*} a W \quad \text { for all } a \in E^{\prime} .
$$

Let $u$ be the standard generator of $C\left(S^{1}\right)$. Denote the quotient map from $B(H)$ onto $B(H) / \mathcal{K}$ by $\pi$ too. Since $\pi \circ \alpha=\pi$,

$$
\|[\pi(W), u]\|=0 .
$$

It follows from Lemma 4.3, there is a continuous path of unitaries $\left\{v_{t}: t \in\right.$ $[0,1]\}$ in $B(H) / \mathcal{K}$ such that $v_{0}=\pi(W), v_{1}=1$ and $\left[v_{t}, u\right]=0$. We claim that there are unitaries $W_{1}, W_{2}, \ldots, W_{N} \in B(H)$ such that

$$
\left\|W_{i}-W_{i+1}\right\|<1 / 2 \text { and } \pi\left(W_{i}\right)=v_{t_{i}},
$$

where $W_{0}=W, i=1,2, \ldots, N$ and $0=t_{0}<t_{1}<\ldots<t_{N}=1$. This claim is shown by applying the following statement repeatly:

If $Z_{1} \in B(H)$ is a unitary, $\pi\left(Z_{1}\right)=z_{1}$ and $z_{2} \in B(H) / \mathcal{K}$ is also a unitary with the property that

$$
\left\|z_{1}-z_{2}\right\|<1 / 4
$$

then there exists a unitary $Z_{2} \in B(H)$ such that

$$
\left\|Z_{1}-Z_{2}\right\|<8\left\|z_{1}-z_{2}\right\| \quad \text { and } \quad \pi\left(Z_{2}\right)=z_{2}
$$

To prove this, we note that there exist unitary $Z_{2}^{\prime} \in B(H)$ with $\pi\left(Z_{2}^{\prime}\right)=z_{2}$ and $a \in \mathcal{K}$ such that

$$
\left\|Z_{1}-\left(Z_{2}^{\prime}+a\right)\right\|<1 / 4
$$

Set $b=Z_{1}^{*}\left(Z_{2}^{\prime}+a\right)$. Then

$$
\|1-b\|<1 / 4 .
$$

So $b$ is invertible. Let $Z_{2}=Z_{1} b\left(b^{*} b\right)^{-1 / 2}$. Note that $\pi(b)=z_{1}^{*} z_{2}$ and $\pi\left(b^{*} b\right)=$ 1 . It is then easily checked that $Z_{2}$ meets the requirement. 
Since $\left[\pi\left(W_{i}\right), u\right]=0, \alpha_{i}(a)=W_{i}^{*} a W_{i}$ for $a \in E^{\prime}$ defines an automorphism on $E^{\prime}$ for $i=0,1, \ldots, N$. Then

$$
\left\|\alpha_{i}-\alpha_{i+1}\right\|<1
$$

for $i=0,1, \ldots, N-1$. Therefore

$$
\left\|\alpha_{i} \circ \alpha_{i+1}^{-1}-\mathrm{id}\right\|<1, \quad i=0,1, \ldots, N-1 .
$$

By $[\mathbf{K R}]$ (see also Theorem 8.7.7 of $[\mathbf{P d}]$ ), $\alpha_{i} \circ \alpha_{i+1}^{-1}$ are derivable automorphisms. We have

$$
\alpha=\left(\alpha \circ \alpha_{1}^{-1}\right) \circ \cdots \circ\left(\alpha_{N-1} \circ \alpha_{N}^{-1}\right) \circ \alpha_{N} .
$$

Since $\pi\left(W_{N}\right)=1, W_{N} \in \tilde{\mathcal{K}}$, the unitization of $\mathcal{K}$. There is a norm continuous path of unitaries in $\tilde{\mathcal{K}}$ which connects $W_{N}$ with 1 . This implies that $\alpha_{N}$ is contained in the connected component of automorphism group of $E^{\prime}$ containing the identity automorphism. It follows from $[\mathbf{K R}]$ (see also 8.7.8 of $[\mathbf{P d}]$ ) that $\alpha_{N}$ is a product of derivable automorphisms. Therefore $\alpha$ is a product of derivable automorphisms of $E^{\prime}$. Since every derivable automorphism is approximately inner (4.2), we conclude that $\alpha$ is approximately inner.

Remark 4.5. It is easy to see that not every automorphism $\alpha$ in 4.4 is inner. Examples can be constructed from our proof of 4.4, using an extension of $C\left(S^{1} \times S^{1}\right)$. The following is a classical example which is provided to us by Jingbo Xia. Identify $\mathcal{T}_{1}$ with the $C^{*}$-algebra generated by Toeplitz operators with continuous symbols. Let $L^{\infty}(\mathbb{T})$ be the $C^{*}$-algebra generated by all Toeplitz operators with symbols $\psi$, where $\psi$ are bounded measurable functions on $\mathbb{T}$. Since $\mathcal{T}_{1} \neq L^{\infty}(\mathbb{T})$, there are unitaries $u \in L^{\infty}(\mathbb{T})$ which are not in $\mathcal{T}_{1}$. However, it is well known that $u x-x u$ is a compact operator for every $x \in \mathcal{T}_{1}$. Let $\alpha: \mathcal{T}_{1} \rightarrow \mathcal{T}_{1}$ by defining $\alpha(x)=u^{*} x u, x \in \mathcal{T}_{1}$ for some $u \in L^{\infty}(\mathbb{T}) \backslash \mathcal{T}_{1}$. Clearly $\alpha$ is an automorphism on $\mathcal{T}_{1}$ with $\pi \circ \alpha=\pi$. It is not inner, since otherwise there would be a unitary $v \in \mathcal{T}_{1}$ such that $v u^{*} x=x v u^{*}$ for each $x \in \mathcal{T}_{1}$ which would imply that $v u^{*}=1$. It would then follow that $u=v \in \mathcal{T}_{1}$. However, it follows from 4.4 that $\alpha$ is approximate inner.

Remark 4.6. Before we typed the paper, we learned from Mikael Rørdam that he had proved, a couple of weeks earlier that an automorphism on $E$ which induces an identity map on the quotient $C\left(S^{1}\right)$ is approximately inner, which is essentially the same result as our 4.4. His nice proof used the Berg's technique. Our proof presented here is completely different and contains other information which is needed in this paper. 
Theorem 4.7. Let $B$ be a unital $C^{*}$-subalgebra of $B(H)$ such that $1_{B}=1$ and $\mathcal{K}=\mathcal{K}(H) \subset B$. Suppose that $\phi_{i}: M_{m}\left(\mathcal{T}_{k}\right) \rightarrow B, i=1,2$ are two unital monomorphisms such that $1-\phi_{i}\left(S_{k}\right) \phi_{i}\left(S_{k}\right)^{*} \in \mathcal{K}$ and $\phi_{1}$ and $\phi_{2}$ induce the same map from $V\left(M_{m}\left(\mathcal{T}_{k}\right)\right)$ into $V(B)$. Then, for any $\varepsilon>0$ and $z_{1}, z_{2}, \ldots, z_{n} \in M_{m}\left(\mathcal{T}_{k}\right)$ which contains the generators $\left\{S_{k}, a_{i j}\right\}$ if $k>0$ or contains $S_{0}$ if $k=0$, there exists $\delta>0$ satisfying the following: If there is a unitary $W \in B$ and $a_{1}, a_{2}, \ldots, a_{n} \in \mathcal{K}(H)$ such that

$$
\left\|W^{*} \phi_{1}\left(z_{i}\right) W-\phi_{2}\left(z_{i}\right)+a_{i}\right\|<\delta, \quad i=1,2, \ldots, n
$$

then there is a unitary $U \in B$ such that

$$
\left\|U^{*} \phi_{1}\left(z_{i}\right) U-\phi_{2}\left(z_{i}\right)\right\|<\varepsilon, \quad i=1,2, \ldots, n .
$$

Furthermore, $\delta$ does not depend on $B$ nor depends on monomorphisms $\phi_{i}$ as long as they satisfy the above conditions.

Proof. Denote $M_{m}\left(\mathcal{T}_{k}\right)$ by $E$. We first assume that $m=1$. To save the notation, we may aslo assume that $W=1$. For the case that $k>0$, without loss of generality, we may further assume that $z_{1}=S_{k}$ and $\left\{z_{2}, \ldots, z_{n}\right\}=\left\{a_{i j}\right\}$ (see 2.1). Let

$$
z_{1}^{\prime}=\left(\phi_{2}\left(z_{1}\right)-a_{1}\right)\left(\left(\phi_{2}\left(z_{1}\right)-a_{1}\right)^{*}\left(\phi_{2}\left(z_{1}\right)-a_{1}\right)\right)^{-1 / 2}
$$

(note that if $\delta<1 / 2,\left(\phi_{2}\left(z_{1}\right)-a_{1}\right)^{*}\left(\phi_{2}\left(z_{1}\right)-a_{1}\right)$ is invertible). Let $p=$ $1-z_{1}^{\prime}\left(z_{1}^{\prime}\right)^{*}$. Then

$$
\left\|\left(1-\phi_{1}\left(z_{1}\right) \phi_{1}\left(z_{1}\right)^{*}\right)-p\right\|<2 \delta .
$$

If $\delta<1 / 4$, there is a unitary $W^{\prime} \in B$ such that

$$
\left\|W^{\prime}-1\right\|<4 \delta \quad \text { and } \quad\left(W^{\prime}\right)^{*}\left(1-\phi_{1}\left(z_{1}\right) \phi_{1}\left(z_{1}\right)\right) W^{\prime}=p .
$$

Define $z_{1+i}^{\prime}=\left(W^{\prime}\right)^{*} \phi_{1}\left(z_{1+i}\right) W^{\prime}, i=1,2, \ldots, n-1$. Then $\left\{z_{1}^{\prime}, z_{2}^{\prime}, \ldots, z_{n}^{\prime}\right\}$ generates a $C^{*}$-subalgebra which is isomorphic to $\mathcal{T}_{k}$ (see 2.1) and

$$
\left\|\phi_{1}\left(z_{i}\right)-z_{i}^{\prime}\right\|<8 \delta \text {. }
$$

Define $\phi_{3}: \mathcal{T}_{k} \rightarrow B$ by sending $z_{i}$ to $z_{i}^{\prime}$.

From the assumption, we see that $\phi_{i}(I(E)) \subset \mathcal{K}(H), i=2,3$. We view $\phi_{3}$ and $\phi_{2}$ as two faithful representations of $E$. The representation $\rho$ of $E$ which maps $I(E)$ onto $\mathcal{K}(H)$ is a faithful irreducible representation of $E$. From representation theory, this is the only faithful irreducible representation (up to unitary equivalence) of $E$ which maps $I(E)$ onto $\mathcal{K}(H)$. 
Suppose that $\phi_{2}\left(1-S_{k} S_{k}^{*}\right)$ has rank $l$. Then $\phi_{1}\left(1-S_{k} S_{k}^{*}\right)$ has rank $l$ too. Therefore, if $\delta$ is small enough, $\phi_{3}\left(1-S_{k} S_{k}^{*}\right)$ has rank $l$. We are going to use Voiculescu's Theorem, as stated in $[\mathbf{A r}]$ (Theorem 5). Let $H_{e}$ be the closure of $\phi_{2}(E) \cap \mathcal{K}(H)(H)$, the essential subspace of $\phi_{2}$. By standard results in representation theory, $\left.\left(\left.\phi_{2}\right|_{I(E)}\right)\right|_{H_{e}}$ is a direct sum of $l$ copies of the faithful irreducible representations of $I(E) \cong \mathcal{K}$. There are $l$ mutually orthogonal rank one projections $d_{1}, d_{2}, \ldots, d_{l}$ in $\mathcal{K}\left(H_{e}\right)$ which commutes with every element in $\left.\phi_{2}(I(E))\right|_{H_{e}}$. Since $\left.\phi_{2}(I(E))\right|_{H_{e}}$ is an essential ideal of $\left.\phi_{2}(E)\right|_{H_{e}}$, $d_{i}$ commutes with every element in $\left.\phi_{2}(E)\right|_{H_{e}}$. Since now $d_{i} \phi_{2}\left(1-S_{k} S_{k}^{*}\right)$ has rank one, $\left.d_{i} \phi_{2}\right|_{H_{e}}$ is irreducible (and also faithful). Thus $\left.\phi_{2}\right|_{H_{e}}$ is unitarily equivalent to $l$ copies of $\rho$. Since $\phi_{3}\left(1-S_{k} S_{k}^{*}\right)$ has also rank $l,\left.\phi_{3}\right|_{H_{e}}$ is also unitarily equivalent to $l$ copies of $\rho$. Therefore the essential parts of $\phi_{2}$ and $\phi_{3}$ are unitarily equivalent. It is trivial that $\operatorname{ker}\left(\pi \circ \phi_{2}\right)=\operatorname{ker}\left(\pi \circ \phi_{3}\right)$. A result of Voiculescu (see Theorem 5 of $[\mathbf{A r}]$ ) now implies that, for any $\varepsilon>0$, there is unitary $V \in B(H)$ such that

$\left\|V^{*} \phi_{2}\left(z_{i}\right) V-\phi_{3}\left(z_{i}\right)\right\|<\varepsilon / 3$ and $V^{*} \phi_{2}\left(z_{i}\right) V-\phi_{3}\left(z_{i}\right) \in \mathcal{K}(H), i=1,2, \ldots, n$.

In particular $V^{*} \phi_{2}\left(z_{i}\right) V \in B$.

Let $E^{\prime}$ be the $C^{*}$-subalgebra of $B$ generated by $\phi_{2}(E)$ and $\mathcal{K}(H)$. Then, since $\pi\left(E^{\prime}\right)=\pi(E), E^{\prime} \cong \mathcal{T}_{k}$. From the construction, $\phi_{2}\left(z_{i}\right)-\phi_{3}\left(z_{i}\right) \in \mathcal{K}(H)$. In fact, since $\left\{z_{2}, z_{3}, \ldots, z_{n}\right\}=\left\{a_{i j}\right\}$ are in $\mathcal{K}(H), z_{i}^{\prime} \in \mathcal{K}(H)$ for $i=2, \ldots, n$. So $\phi_{2}\left(z_{i}\right)-\phi_{3}\left(z_{i}\right) \in \mathcal{K}(H)$ for $i=2,3, \ldots, n$. We also (note $a_{1} \in \mathcal{K}(H)$ ) have

$$
\pi\left(z_{1}^{\prime}\right)=\pi \circ \phi_{2}\left(z_{1}\right) \pi\left(\left(\left(\phi_{2}\left(z_{1}\right)-a_{1}\right)^{*}\left(\phi_{2}\left(z_{1}\right)-a_{1}\right)\right)^{1 / 2}\right)=\pi \circ \phi_{2}\left(z_{1}\right),
$$

where $\pi: B(H) \rightarrow B(H) / \mathcal{K}(H)$ is the quotient map. Thus $\phi_{2}\left(z_{i}\right)-\phi_{3}\left(z_{i}\right) \in$ $\mathcal{K}(H)$ for all $i$. Therefore $A d(V)$ gives an automorphism on $E^{\prime}$. Furthermore, $\pi \circ A d(V)=\pi$ on $E^{\prime}$. By 4.5 , there is a unitary $U \in E^{\prime} \subset B$ such that

$$
\left\|U \phi_{2}\left(z_{i}\right) U^{*}-V^{*} \phi_{2}\left(z_{i}\right) V\right\|<\varepsilon / 3, \quad i=1,2, \ldots, n .
$$

Thus

$$
\begin{aligned}
& \left\|U^{*} \phi_{1}\left(z_{i}\right) U-\phi_{2}\left(z_{i}\right)\right\| \\
& \leq\left\|U^{*} \phi_{1}\left(z_{i}\right) U-U^{*} \phi_{3}\left(z_{i}\right) U\right\|+\left\|U^{*} \phi_{3}\left(z_{i}\right) U-U^{*} V^{*} \phi_{2}\left(z_{i}\right) V U\right\| \\
& \quad+\left\|U^{*} V^{*} \phi_{2}\left(z_{i}\right) V U-\phi_{2}\left(z_{i}\right)\right\|<8 \delta+\varepsilon / 3+\varepsilon / 3<\varepsilon,
\end{aligned}
$$

if $8 \delta<\varepsilon / 3$.

We now consider the case that $k=0$. Although we may not need this in our proof of the classification theorems, it is of interesting on its own. Note 
that $S_{0}$ is a unitary and there is an approximate identity $\left\{p_{m}\right\}$ for $I\left(\mathcal{T}_{0}\right)$ consisting of projections such that

$$
\left\|p_{m} S_{0}-S_{0} p_{m}\right\| \rightarrow 0
$$

With an error within $\varepsilon / 8$, we may assume that

$$
z_{i} \in p_{N} I\left(\mathcal{T}_{0}\right) p_{N}, \quad i=2,3, \ldots, n
$$

and

$$
\left\|p_{N} S_{0}-S_{0} p_{N}\right\|<\varepsilon / 8
$$

for some integer $N$. There is a unitary $s \in\left(1-p_{N}\right) \mathcal{T}_{0}\left(1-p_{N}\right)$ such that

$$
\left\|s-\left(1-p_{N}\right) S_{0}\left(1-p_{N}\right)\right\|<\varepsilon / 8 .
$$

So, without loss of generality, we may assume that, in this case, $z_{1}=s \in$ $\left(1-p_{N}\right) \mathcal{T}_{0}\left(1-p_{N}\right), z_{2}, z_{3}, \ldots, z_{n} \in p_{N} I\left(\mathcal{T}_{0}\right) p_{N}$ (we may have to consider $n$ elements in $p_{N} I\left(\mathcal{T}_{0}\right) p_{N}$ instead of $(n-1)$ elements, but we can change notation). Note that $\left(1-p_{N}\right) \mathcal{T}_{0}\left(1-p_{N}\right) \cong \mathcal{T}_{0}$. Since $\phi_{1}$ and $\phi_{2}$ induce the same map on $V\left(\mathcal{T}_{0}\right), \phi_{1}\left(p_{N}\right)$ is equivalent to $\phi_{2}\left(p_{N}\right)$. So there is a unitary $W_{1} \in B$ such that

$$
W_{1}^{*} \phi_{1}\left(p_{N}\right) W_{1}=\phi_{2}\left(p_{N}\right) .
$$

Without loss of generality, we may assume that $\phi_{1}\left(p_{N}\right)=\phi_{2}\left(p_{N}\right)$. The argument used in the case that $k>0$ shows that there is a unitary $U^{\prime} \in$ $\phi_{1}\left(1-p_{N}\right) B \phi_{1}\left(1-p_{N}\right)$ such that

$$
\left\|\left(U^{\prime}\right)^{*} \phi_{1}\left(z_{1}\right) U^{\prime}-\phi_{2}\left(z_{1}\right)\right\|<\varepsilon / 8 .
$$

Since $z_{2}, z_{3}, \ldots, z_{n}$ generate a finite dimensional $C^{*}$-subalgebra of $p_{N} I\left(\mathcal{T}_{k}\right) p_{N}$ and $\phi_{1}$ and $\phi_{2}$ induce the same map on $V\left(\mathcal{T}_{0}\right)$, there is a unitary $u_{1} \in$ $\phi_{1}\left(p_{N}\right) \mathcal{K}(H) \phi_{1}\left(p_{N}\right)$ such that

$$
u_{1}^{*} \phi_{1}\left(z_{i}\right) u_{1}=\phi_{2}\left(z_{i}\right), \quad i=2,3, \ldots, n .
$$

Now set $U=U^{\prime}+u_{1}$. Then

$$
\left\|U^{*} \phi_{1}\left(z_{i}\right) U-\phi_{2}\left(z_{i}\right)\right\|<\varepsilon, i=1,2, \ldots, n .
$$

For the case that $m>0$, We first find a unitary $Z \in B$ such that $A d(Z) \circ \phi_{1}$ agrees with $\phi_{2}$ on $M_{m}(\mathbf{C})$. So we may wssume that $Z=1$. Note then that $\phi_{1}\left(e_{11}\right) \mathcal{K} \phi_{1}\left(e_{11}\right) \cong \mathcal{K}$. We then consider $\phi_{1}\left(e_{11}\right) \phi_{1}(-) \phi_{1}\left(e_{11}\right)$, $\phi_{2}\left(e_{11}\right) \phi_{2}(-) \phi_{2}\left(e_{11}\right)$ and $\phi_{1}\left(e_{11}\right) B \phi_{1}\left(e_{11}\right)$. So the case that $m>1$ is reduced to the case that $m=1$. 
Finally, from the proof, we see that our $\delta$ is independent of $C^{*}$-algebra $B$ and $\phi_{i}$ as long as they satisfy the conditions of the theorem.

Corollary 4.8. Let $B$ be as in 4.7. Suppose that $\phi_{i}: M_{m}\left(C\left(S^{1}\right)\right) \rightarrow B, i=$ 1,2 are two unital monomorphisms such that $\left[\phi_{1}\left(e_{11}\right)\right]=\left[\phi_{2}\left(e_{11}\right)\right]$ in $V(B)$, where $\left\{e_{i j}\right\}_{i, j=1}^{m}$ is a matrix unit for $M_{m}(\mathbf{C})$. Then, for any $\varepsilon>0$ there exists $\delta>0$ satisfying the following: If there is unitary $W \in B$ and $a_{0}, a_{i j} \in \mathcal{K}$ such that

$$
\left\|W^{*} \phi_{1}(u) W-\phi_{2}(u)+a_{0}\right\|<\delta
$$

and

$$
\left\|W^{*} \phi_{1}\left(e_{i j}\right) W-\phi_{2}\left(e_{i j}\right)+a_{i j}\right\|<\delta
$$

then there is unitary $U \in B$ such that

$$
\left\|U^{*} \phi_{1}(u) U-\phi_{2}(u)\right\|<\varepsilon
$$

and

$$
\left\|U^{*} \phi_{1}\left(e_{i j}\right) U-\phi_{2}\left(e_{i j}\right)\right\|<\varepsilon,
$$

where $i, j=1,2, \ldots, n$ and $u$ is the standard generator of $C\left(S^{1}\right)$.

Proof. Let us first consider the case that $m=1$. Let $D$ be the $\mathrm{C}^{*}$-subalgebra of $B$ generated by $\phi_{2}(u)$ and $\mathcal{K}$. Then $D$ is isomorphic to $\mathcal{T}_{0}$ and $\phi_{2}(u)-a_{0} \in$ $D$. As in the proof of 4.7 , one shows that there is a unitary $W \in D$ such that $W \phi_{2}(u) W^{*}$ and $\phi_{2}(u)-a_{0}$ are close. The result then follows.

For the case $m>1$, the proof is the same as that of 4.7 . It can be reduced to the case that $m=1$.

4.9. The following definition is taken from [EG2].

Definition. Let $A=B \otimes C\left(S^{1}\right)$, where $B$ is a finite dimensional $C^{*}$ algebra and let $\epsilon$ be a positive number. A finite subset $F \subset A$ is weakly approximately constant to within $\epsilon$ if for any $t \in S^{1}$ there exists a unitary $U(t) \in B$ such that $\operatorname{Ad}(U(t)) f(t)$ and $f(1)$ is within $\epsilon$ for every $f \in F$, i.e.

$$
\|\operatorname{Ad}(U(t)) f(t)-f(1)\|<\varepsilon \quad \text { for } f \in F .
$$

Let $A$ and $B$ be two (unital) $C^{*}$-algebras, let $F \subset A$ be a subset and let $\phi, \psi: A \rightarrow B$ two homomorphisms. We say $\phi$ and $\psi$ are approximately unitarily equivalent on $F$ to within $\varepsilon$ if there is a unitary $u \in B$ such that

$$
\|\operatorname{Ad} u \circ \phi(f)-\psi(f)\|<\varepsilon \quad \text { for } f \in F \text {. }
$$


Theorem 4.10. Let $E_{1}=M_{n}\left(\mathcal{T}_{k}\right)$ and $E_{2}=M_{m}\left(\mathcal{T}_{r}\right)$ be two $\mathcal{T}$-algebras, let $\pi_{1}$ be the quotient map from $E_{1}$ to $Q\left(E_{1}\right)$, let $F \subset E_{1}$ be a finite subset such that $\pi_{1}(F)$ contains the standard generators of $Q\left(E_{1}\right)$, and let $\phi$ and $\psi: E_{1} \rightarrow E_{2}$ be two *-homomorphisms which induce the same map on $V_{*}\left(E_{1}\right)$. For given $\epsilon>0$, there exists $\delta>0$ only depending on $E_{1}, F$ and $\epsilon$ such that the followings hold:

(1) If $\phi$ and $\psi$ are injective and if the two induced maps $Q(\phi)$ and $Q(\psi)$ from $Q\left(E_{1}\right)$ to $Q\left(E_{2}\right)$ are approximately the same to within $\delta$ on $\pi_{1}(F) \subset Q\left(E_{1}\right)$, then $\phi$ and $\psi$ are approximately unitarily equivalent on $F$ to within $\epsilon$.

(2) If $\phi$ and $\psi$ are not injective but the two maps $\phi_{Q}$ and $\psi_{Q}$ induced by $\phi$ and $\psi$ from $Q\left(E_{1}\right)$ to $E_{2}$ are injective, and if $Q(\phi)$ and $Q(\psi)$ are approximately the same on $\pi_{1}(F)$ to within $\delta$, then $\phi$ and $\psi$ are approximately unitarily equivalent on $F$ to within $\epsilon$.

Proof. (1) Since $\phi$ and $\psi$ induce the same map on $V_{*}\left(E_{1}\right)$, there exists a unitary $X \in E_{2}$ such that $\phi$ and $\operatorname{Ad} X \circ \psi$ agree on $M_{n}(\mathbf{C}) \subset M_{n}\left(\mathcal{T}_{k}\right)$. So we may assume that they agree on $M_{n}(\mathbf{C})$. Denote $\phi(1)=\psi(1)=p$, and denote $B=p E_{2} p$.

Fix $\epsilon>0$ and $F$ as above. If $Q(\phi)$ and $Q(\psi)$ are approximately the same on $\pi_{1}(F)$ to within $\delta$, there exist $W=1$ and $a_{f}$ to satisfy the conditions of Theorem 4.7. It follows that there exists a unitary $U \in B$ such that

$$
\left\|U^{*} \phi(f) U-\psi(f)\right\|<\epsilon \quad f \in F .
$$

This completes the proof of (1).

(2) Since $\phi$ and $\psi$ are not injective, they vanish on $I\left(E_{1}\right)$. Now $\phi_{Q}$ and $\psi_{Q}$ are defined. By the assumption, they are injective. Since $\phi=\phi_{Q} \circ \pi_{1}$ and $\psi=\psi_{Q} \circ \pi_{1}$ and since $\pi_{1}$ induces an onto map from $V\left(E_{1}\right)$ to $V\left(Q\left(E_{1}\right)\right), \phi_{Q}$ and $\psi_{Q}$ induce the same map on $V\left(Q\left(E_{1}\right)\right)$.

Since $Q(\phi)$ and $Q(\psi)$ are approximately the same on $\pi_{1}(F)$ to within $\delta, \phi_{Q}$ and $\psi_{Q}$ are approximately unitarily equivalent on $\pi_{1}(F)$ to within $\epsilon$ (Corollary 4.8). Since $\phi_{Q} \circ \pi_{1}=\phi$ and $\psi_{Q} \circ \pi_{1}=\psi, \phi$ and $\psi$ are approximately unitarily equivalent on $F$ to within $\epsilon$.

This completes the proof of the theorem.

Theorem 4.11. Let $E_{1}=M_{n}\left(\mathcal{T}_{k}\right)$ and $E_{2}=M_{m}\left(\mathcal{T}_{r}\right)$ be two $\mathcal{T}$-algebras, let $\pi_{1}$ be the quotient map from $E_{1}$ to $Q\left(E_{1}\right)$, let $\epsilon>0$, let $F \subset E_{1}$ be a finite subset such that $\pi_{1}(F)$ is of weakly approximately constant to within 
$\epsilon / 2$, and let $\phi$ and $\psi: E_{1} \rightarrow E_{2}$ be two $*$-homomorphisms which induce the same map on $V_{*}\left(E_{1}\right)$. Then the followings hold:

(1) If $\phi$ and $\psi$ are not injective, the images of $\phi_{Q}(u)$ and $\psi_{Q}(u)$ are of finite spectra, where $\phi_{Q}$ and $\psi_{Q}$ are the $*$-homomorphisms from $Q\left(E_{1}\right)$ to $E_{2}$ induced by $\phi$ and $\psi$, respectively, and where $u$ is the canonical generator of $e_{11} M_{n}\left(C\left(S^{1}\right)\right) e_{11}\left(\left\{e_{i j}\right\}\right.$ is the matrix unit for $\left.M_{n}(\mathbf{C})\right)$, then $\phi$ and $\psi$ are approximately unitarily equivalent on $F$ to within $\epsilon$.

(2) If $Q(\phi)$ and $Q(\psi)$, the *-homomorphisms from $Q\left(E_{1}\right)$ to $Q\left(E_{2}\right)$ induced by $\phi$ and $\psi$, respectively, are zero, then $\phi$ and $\psi$ are approximately unitarily equivalent on $F$ to within $\epsilon$.

Proof. (1) Since $\phi$ and $\psi$ induce the same map on $V_{*}\left(E_{1}\right)$, by conjugating a unitary, if necessary, we may assume that $\phi$ and $\psi$ agree on $M_{n}(\mathbf{C})$. In particular, $\phi(1)=\psi(1)=p \in E_{2}$. Let $B=p E_{2} p$. By assumption, $\phi_{Q}(u)=$ $\sum a_{t} p_{t}^{(1)}$ where $a_{t} \in S^{1}$ and where $p_{t}^{(1)}$ are finitely many mutually orthogonal projections in $B$. Let $p_{t}^{(i)}=\phi_{Q}\left(e_{i 1}\right) p_{t}^{(1)} \phi_{Q}\left(e_{1 i}\right), p_{k}=\sum_{i=1}^{m} p_{k}^{(i)}$ and $p=$ $\sum p_{k}=\phi(1) \in B$, where $\left\{e_{i j}\right\}$ is the matrix unit of $M_{n}(\mathbf{C})$. For any $h \in$ $Q\left(E_{1}\right) \cong M_{n}\left(C\left(S^{1}\right)\right)$, we have

$$
\phi_{Q}(h)=\sum_{k} \phi_{Q}\left(h\left(a_{k}\right)\right) p_{k}
$$

Similarly, we have

$$
\psi_{Q}(h)=\sum_{j} \psi_{Q}\left(h\left(b_{j}\right)\right) q_{j}
$$

where $\sum q_{j}=p$, and where $b_{k} \in S^{1}$.

Since $\pi_{1}(F)$ is weakly approximately constant to within $\epsilon / 2$, there exist unitaries $X_{i}, Y_{i} \in M_{n}(\mathbf{C})$ such that

$$
\begin{array}{r}
\left\|X_{i} \pi_{1}(f)\left(a_{i}\right) X_{i}^{*}-X_{1} \pi_{1}(f)\left(a_{1}\right) X_{1}^{*}\right\|<\epsilon / 2 \\
\left\|Y_{i} \pi_{i}(f)\left(b_{i}\right) Y_{i}^{*}-Y_{1} \pi_{1}(f)\left(a_{1}\right) Y_{1}^{*}\right\|<\epsilon / 2 .
\end{array}
$$

Write

$$
U=\sum \phi_{Q}\left(X_{k}^{*} X_{1}\right) p_{k}
$$

Then $U$ is a unitary in $B$ and

$$
\left\|\phi_{Q}\left(\pi_{1}(f)\right)-U^{*} \phi_{Q}\left(\pi_{1}(f)\left(a_{1}\right)\right) U\right\|<\epsilon / 2 \quad f \in F .
$$

Similarly, we have

$$
\left\|\psi_{Q}\left(\pi_{1}(f)\right)-V^{*} \psi_{Q}\left(\pi_{1}(f)\left(a_{1}\right)\right) V\right\|<\epsilon / 2 \quad f \in F
$$


where $V=\sum \psi_{Q}\left(Y_{j}^{*} Y_{1}\right) q_{j}$ is a unitary in $B$. Note by the assumption at the beginning of this part of the proof, $\phi_{Q}$ agrees with $\psi_{Q}$ on $M_{n}(\mathbf{C})$. Thus

$$
\phi_{Q}\left(\pi_{1}(f)\left(a_{1}\right)\right)=\psi_{Q}\left(\pi_{1}(f)\left(a_{1}\right)\right) .
$$

Hence

$$
\left\|V U^{*} \phi_{Q}\left(\pi_{1}(f)\right) U V^{*}-\psi_{Q}\left(\pi_{1}(f)\right)\right\|<\epsilon \quad f \in F .
$$

Notice that $\phi=\phi_{Q} \circ \pi_{1}$ and $\psi=\psi_{Q} \circ \pi_{1}$, we have

$$
\left\|V U^{*} \phi(f) U V^{*}-\psi(f)\right\|<\epsilon \quad f \in F .
$$

(2) Again, as in (1), we may assume that $\phi$ and $\psi$ agree on $M_{n}(\mathbf{C})$. We also know that $\phi$ and $\psi$ factor through $Q\left(E_{1}\right)$. Therefore $\phi_{Q}$ and $\psi_{Q}$ are two maps from $Q\left(E_{1}\right)$ to $I\left(E_{2}\right)$. Denote $p=\phi_{Q}(1)=\psi_{Q}(1)$. We have $p I\left(E_{2}\right) p \cong M_{l}$ where $l=[p] \in \mathbf{Z}_{+}$. So $\phi_{Q}$ and $\psi_{Q}$ are two unital $*$-homomorphisms from $Q\left(E_{1}\right)$ to $p I\left(E_{2}\right) p \cong M_{l}$. We may write

$$
\phi_{Q}(h)=\sum_{k} \phi_{Q}\left(h\left(a_{k}\right)\right) p_{k} \quad \text { and } \quad \psi_{Q}(h)=\sum_{j} \psi_{Q}\left(h\left(b_{j}\right)\right) q_{j},
$$

for $h \in M_{n}\left(C\left(S^{1}\right)\right)$, where $a_{k}, b_{j} \in S^{1},\left\{p_{k}\right\}$ and $\left\{q_{j}\right\}$ are mutually orthogonal projections in $p I\left(E_{2}\right) p$ and $\sum_{k} p_{k}=\sum_{j} q_{j}=p$.

Since $\pi_{1}(F)$ is weakly approximately constant to within $\epsilon / 2$, the same argument used in the proof of (1) shows that $\phi_{Q}$ and $\psi_{Q}$ are approximately unitarily equivalent on $\pi_{1}(F)$ to within $\epsilon$. Since $\phi=\phi_{Q} \circ \pi_{1}$ and $\psi=\psi_{1} \circ \pi_{1}$, $\phi$ and $\psi$ are approximately unitarily equivalent on $F$ to within $\epsilon$.

\section{The Classification.}

The following is the main result of this paper.

Theorem 5.1. Let $E$ and $E^{\prime}$ be two unital $C^{*}$-algebras in $\mathbb{I}$. Suppose that $\eta: V_{*}(E) \longrightarrow V_{*}\left(E^{\prime}\right)$ is an isomorphism such that $\eta\left(\left[1_{E}\right]\right)=\left[1_{E^{\prime}}\right]$. Then there exists $a *$-isomorphism $\phi: E \longrightarrow E^{\prime}$ such that $\phi$ induces $\eta$.

The theorem is a direct consequence of Lemma 5.4 and Proposition 5.7 below.

We note that if $\phi: E \rightarrow E^{\prime}$ is an isomorphism, then $\phi_{*}$ induces an isomorphism from $V_{*}(E)$ onto $V_{*}\left(E^{\prime}\right)$. Therefore, together with 3.7, we give a complete classification of unital $C^{*}$-algebras in $\mathbb{T}$.

Write $E=\lim _{\rightarrow}\left(E_{n}, \phi_{n, n+1}\right)$ and $E^{\prime}=\lim _{\rightarrow}\left(E_{n}^{\prime}, \psi_{n, n+1}\right)$. One would hope that $\eta$ induces an intertwining between the two sequences $\left\{V_{*}\left(E_{n}\right)\right\}$ and 
$\left\{V_{*}\left(E_{m}^{\prime}\right)\right\}$ for each $n$. However, since $V\left(\mathcal{T}_{0}\right)$ is not finitely generated, it may not be possible. To overcome this difficulty, we first impose a condition on the connecting maps $\phi_{n, n+1}$ and $\psi_{n, n+1}$ (see Lemma 5.4 below). We then show that the general case can be reduced to this case.

We will consider unital $C^{*}$-algebras only. Therefore, in what follows, we will always assume that all connecting maps in an inductive limit system are unital.

5.2. The following is essentially Theorem 6.2 of [Ell2].

Theorem. Let $A=\lim _{\rightarrow} A_{n}$ be a $C^{*}$-algebra of real rank zero where each $A_{n}$ is a finite direct sum of matrix algebras over $C\left(S^{1}\right)$. Then for any $\epsilon>0$, for any $n$, and for any finite subset $F \subset A_{n}$, there exists $m>n$ such that the image of $F$ in $A_{k}$ for $k \geq m$ is weakly approximately constant to within $\epsilon$.

5.3. The following is Theorem 2.29 of [EG2]. We only state it for the special case that we will use later.

Theorem. Let $A=\lim _{\rightarrow}\left(A_{n}, \phi_{n, n+1}\right)$ be a $C^{*}$-algebra of real rank zero where each $A_{n}$ is a finite direct sum of matrix algebras over $C\left(S^{1}\right)$, let $\epsilon>0$ and let $F \subset A_{n}$ be a finite subset of weakly approximately constant to within $\epsilon$. If $\phi$ and $\psi$ are two $*$-homomorphisms from $A_{n}$ to $A_{n+1}$ which induce the same $K$-theory maps, then there exists $m>n$ such that $\phi_{n, k} \circ \phi$ and $\phi_{n, k} \circ \psi$ are approximately unitarily equivalent on $F$ to within $80 \epsilon$ for all $k \geq m$.

Lemma 5.4. Let $E$ and $E^{\prime}$ be two unital $C^{*}$-algebras in $\mathbb{T}$. Suppose that each connecting map in the two inductive limit systems satisfies the following: each partial map of a connecting map from $M_{n}\left(\mathcal{T}_{0}\right)$ vanishes on the ideal $M_{n}(\mathcal{K}) \subset M_{n}\left(\mathcal{I}_{0}\right)$. Suppose that $\eta: V_{*}(E) \longrightarrow V_{*}\left(E^{\prime}\right)$ is an isomorphism such that $\eta\left(\left[1_{E}\right]\right)=\left[1_{E^{\prime}}\right]$, then there exists a $*$-isomorphism $\phi: E \longrightarrow E^{\prime}$ such that $\phi$ induces $\eta$.

Proof. Write $E=\lim _{\rightarrow}\left(E_{n}, \phi_{n, n+1}\right)$ and $E^{\prime}=\lim _{\rightarrow}\left(E_{n}^{\prime}, \psi_{n, n+1}\right)$ where each $E_{n}$ or $E_{n}^{\prime}$ is a finite direct sums of matrix algebras over $\mathcal{T}$-algebras. We will construct an approximate intertwining between these two sequences.

But first we intertwine $V_{*}$.

After passing to the subsequences and changing the notation, by 2.14, the isomorphism between $V_{*}(E)$ and $V_{*}\left(E^{\prime}\right)$ gives the following commutative diagram:

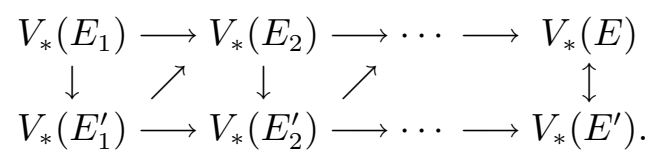


This diagram induces a commutative diagram

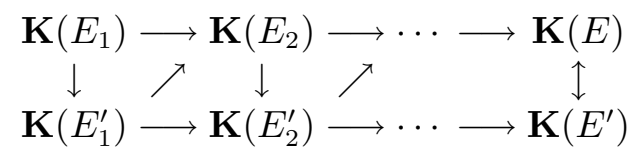

(Lemma 2.14). Furthermore, this diagram gives the following commuting diagram:

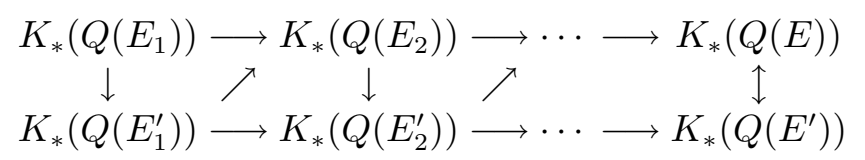

such that each map preserves the order.

By the existence Theorem 3.1, there are unital $*$-homomorphisms $\alpha_{k}$ and $\beta_{k}$ from $E_{k}$ to $E_{k}^{\prime}$ and from $E_{k}^{\prime}$ to $E_{k+1}$ that induce the above diagrams Notice that the diagram:

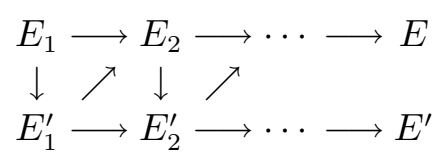

is not necessarily commutative. We will turn this diagram into an approximate intertwining diagram:

$$
\begin{aligned}
& E_{n_{1}} \longrightarrow E_{n_{2}} \longrightarrow \cdots \longrightarrow E \\
& \alpha_{1} \downarrow \beta_{1} \nearrow \alpha_{2} \downarrow \beta_{2} \nearrow \\
& E_{m_{1}}^{\prime} \longrightarrow E_{m_{2}}^{\prime} \longrightarrow \cdots \longrightarrow E^{\prime}
\end{aligned}
$$

as follows.

First, some conventions. By the generators of $M_{n}\left(C\left(S^{1}\right)\right)$, we mean the set $\left\{e_{i j}\right\}$ and the function $z$, where $\left\{e_{i j}\right\}$ is a matrix unit for $M_{n}(\mathbf{C})$ and $z \in C\left(S^{1}\right)$ which is the identity map on $S^{1}$. Furthermore, we view $z \in$ $e_{11} M_{n}\left(C\left(S^{1}\right)\right) e_{11}$.

Let $H_{1}$ and $H_{2}$ be two finite direct sums of matrix algebras over $\mathcal{T}$ algebras. Let $\phi$ from $H_{1}$ to $H_{2}$ be a $*$-homomorphism. Then $\phi$ induces uniquely a $*$-homomorphism from $Q\left(H_{1}\right)$ to $Q\left(H_{2}\right)$. We will denote this map by $Q(\phi)$. We will denote by $\pi_{i}$ and $\pi_{i}^{\prime}$ the quotient maps from $E_{i}$ to $Q\left(E_{i}\right)$ and from $E_{i}^{\prime}$ to $Q\left(E_{i}^{\prime}\right)$, respectively.

Let $\left\{\epsilon_{n}\right\}$ and $\left\{\epsilon_{n}^{\prime}\right\}$ be two sequences of decreasing positive numbers with finite summations. Let $\Delta_{i}=\left\{f_{n}^{(i)}\right\} \subset E_{i}$ and $\Delta_{i}^{\prime}=\left\{f_{n}^{(i)^{\prime}}\right\} \subset E_{i}^{\prime}$ be dense for all $i$. Let $F_{1} \subset E_{1}$ be a finite subset such that $F_{1}$ contains $f_{1}^{(1)}$ and $\pi_{1}\left(F_{1}\right) \subset Q\left(E_{1}\right)$ contains the generators of $Q\left(E_{1}\right)$. For $\epsilon_{1}>0$, by Theorem 
5.2 , there exists $n_{1}$ such that $\pi_{n_{1}}\left(\phi_{1, n_{1}}\left(F_{1}\right)\right)$ is weakly approximately constant to within $\epsilon_{1} / 2$. We will denote $\phi_{1, n_{1}}\left(F_{1}\right) \subset E_{n_{1}}$ by $F_{1}$ again.

We want to find $m_{1}>n_{1}$ and construct a unital $*$-homomorphism from $E_{n_{1}}$ to $E_{m_{1}}^{\prime}$. The map we are going to construct takes the form $\psi_{k\left(n_{1}\right), m_{1}} \circ$ $\alpha_{k\left(n_{1}\right)} \circ \phi_{n, k\left(n_{1}\right)}$, where $k\left(n_{1}\right)>n_{1}$ :

$$
\begin{aligned}
E_{n_{1}} & \longrightarrow E_{k\left(n_{1}\right)} \\
\downarrow & \downarrow \\
& E_{k\left(n_{1}\right)}^{\prime} \longrightarrow E_{m_{1}}^{\prime} .
\end{aligned}
$$

We now specify the choice of $k\left(n_{1}\right)$ and $m_{1}$. Let $\Gamma_{1}$ be a finite subset of $E_{n_{1}}$ such that $\Gamma_{1}$ contains $F_{1}$ and $\pi_{n_{1}}\left(\Gamma_{1}\right)$ contains the generators of $Q\left(E_{n_{1}}\right)$. For $E_{n_{1}}, \Gamma_{1}$ and $\epsilon_{1} / 2$, there exists $\delta_{1}$ to satisfy the conclusions (1) and (2) of Theorem 4.10. Let $G_{1}=\pi_{n_{1}}\left(\Gamma_{1}\right)$. By Theorem 5.2, there exists $k\left(n_{1}\right)>n_{1}$ such that $Q\left(\phi_{n_{1}, k\left(n_{1}\right)}\right)\left(G_{1}\right)$ is of weakly approximately constant to within $\delta_{1} / 160$. Recall that $\alpha_{k\left(n_{1}\right)}$ is a unital $*$-homomorphism from $E_{k\left(n_{1}\right)}$ to $E_{k\left(n_{1}\right)}^{\prime}$. Let $F \subset E_{n_{1}}$ be a finite subset which contains the images of the first $n_{1}$ elements of $\Delta_{1}, \cdots, \Delta_{\left(n_{1}-1\right)}$ and $\Delta_{n_{1}}$. We also require that $\pi_{n_{1}}(F)$ contains the generators of $Q\left(E_{n_{1}}\right)$ and the image of $F_{1}$. By Theorem 5.2 again, there exists $m_{1}>k\left(n_{1}\right)$ such that $\pi_{m_{1}}^{\prime} \circ \psi_{k\left(n_{1}\right), m_{1}} \circ \alpha_{k\left(n_{1}\right)} \circ \phi_{n_{1}, k\left(n_{1}\right)}(F) \subset Q\left(E_{m_{1}}^{\prime}\right)$ is of weakly approximately constant to within $\epsilon_{1}^{\prime} / 2$. We will denote $\psi_{k\left(n_{1}\right), m_{1}} \circ$ $\alpha_{k}\left(n_{1}\right) \circ \phi_{n_{1}, k\left(n_{1}\right)}(F)$ by $F_{1}^{\prime}$. We have a map $\psi_{k\left(n_{1}\right), m_{1}} \circ \alpha_{k}\left(n_{1}\right) \circ \phi_{n_{1}, k\left(n_{1}\right)}$ from $E_{n_{1}}$ to $E_{m_{1}}^{\prime}$.

Next, we will construct a map from $E_{m_{1}}^{\prime}$ to $E_{n_{2}}$ for some $n_{2}>m_{1}$. The map will take the following form:

$$
E_{m_{1}}^{\prime} \longrightarrow E_{k\left(m_{1}\right)}^{\prime} \nearrow^{E_{k\left(m_{1}\right)+1} \longrightarrow E_{l} \longrightarrow E_{n_{2}}}
$$

where $l>k\left(m_{1}\right)+1$. Let $\Gamma_{1}^{\prime}$ be a finite subset of $E_{m_{1}}^{\prime}$ such that $\Gamma_{1}^{\prime}$ contains $F_{1}^{\prime}$ and $\pi_{m_{1}}^{\prime}\left(\Gamma_{1}^{\prime}\right)$ contains the generators of $Q\left(E_{m_{1}}^{\prime}\right)$. For $E_{m_{1}}^{\prime}, \Gamma_{1}^{\prime}$ and $\epsilon_{1}^{\prime} / 2$, there exists $\delta_{1}^{\prime}$ to satisfy the conclusions (1) and (2) of Theorem 4.10. Let $k\left(m_{1}\right)>m_{1}$ so that $Q\left(\psi_{m_{1}, k\left(m_{1}\right)}\right)\left(G_{1}^{\prime}\right)$ is of weakly approximately constant to within $\delta_{1}^{\prime} / 160$. Here $G_{1}^{\prime}=\pi_{m_{1}}^{\prime}\left(\Gamma_{1}^{\prime}\right)$. Recall that there is a unital *homomorphism $\beta_{k\left(m_{1}\right)}$ from $E_{k\left(m_{1}\right)}^{\prime}$ to $E_{k\left(m_{1}\right)+1}^{\prime}$. By Theorem 5.3, there exists $l>k\left(m_{1}\right)$ such that $Q\left(\phi_{k\left(n_{1}\right), l}\right)$ and $Q\left(\phi_{k\left(m_{1}\right)+1, l} \circ \beta_{k\left(m_{1}\right)} \circ \psi_{k\left(n_{1}\right), k\left(m_{1}\right)} \circ \alpha_{k\left(n_{1}\right)}\right)$ are approximately unitarily equivalent on $Q\left(\phi_{n_{1}, k\left(n_{1}\right)}\right)\left(G_{1}\right)$ to within $\delta_{1} / 2$.

We are now in the following situation. There is a not necessarily commutative diagram:

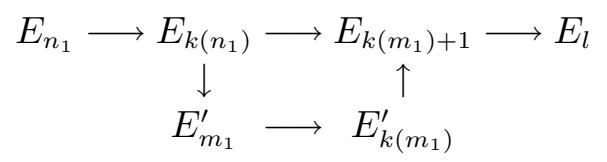


where the down map is $\psi_{k\left(n_{1}\right), m_{1}} \circ \alpha_{k\left(n_{1}\right)} \circ \phi_{n_{1}, k\left(n_{1}\right)}$ and the up map is $\beta_{k\left(m_{1}\right)}$. Here $\pi_{n_{1}}\left(F_{1}\right) \subset Q\left(E_{n_{1}}\right)$ is of weakly approximately constant to within $\epsilon_{1} / 2$, $\pi_{m_{1}}^{\prime}\left(F_{1}^{\prime}\right) \subset Q\left(E_{m_{1}}^{\prime}\right)$ is of weakly approximately constant to within $\epsilon_{1}^{\prime} / 2$ and, by 5.3 , the following diagram is approximately commutative, up to an inner automorphism on $Q\left(E_{l}\right)$, on $G_{1}$ to within $\delta_{1} / 2$ :

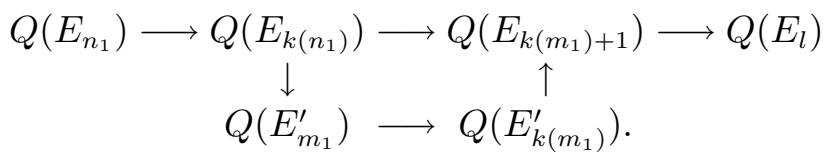

Denote by $\beta_{k\left(m_{1}\right)}^{\prime}$ be the composition of a suitable inner automorphism $\operatorname{Ad}(u)$ on $Q\left(E_{l}\right)$ with the composition of the map from $Q\left(E_{k\left(m_{1}\right)}^{\prime}\right)$ to $Q\left(E_{k\left(m_{1}\right)+1}\right)$ with the map from $Q\left(E_{k\left(m_{1}\right)+1}\right)$ to $Q\left(E_{l}\right)$ in the above diagram. We choose the inner automorphism so that the following diagram approximately commutative on $G_{1}$ within $\delta_{1} / 2$ :

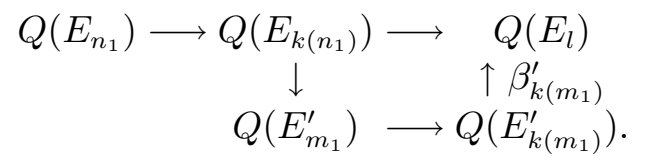

If $\Phi$ is the map from $E_{k\left(m_{1}\right)}^{\prime}$ to $E_{l}$ obtained from the diagram marked with $(\star)$ in this proof, then

$$
\operatorname{Ad}(u) \circ Q(\Phi)=\beta_{k\left(m_{1}\right)}^{\prime} .
$$

By the existence theorem (3.1), there is a map $\Psi: E_{k\left(m_{1}\right)}^{\prime} \rightarrow E_{l}$ such that $Q(\Psi)=\beta_{k\left(m_{1}\right)}^{\prime}$ and $\Psi$ and $\Phi$ induces the same map on $V_{*}\left(E_{k\left(m_{1}\right)}^{\prime}\right)$. (The use of $\Psi$ is to avoid to lift $u$.)

We now denote by $\alpha$ the composition of the maps from $E_{n_{1}}$ to $E_{k\left(n_{1}\right)}$, from $E_{k\left(n_{1}\right)}$ to $E_{m_{1}}^{\prime}$, from $E_{m_{1}}^{\prime}$ to $E_{k\left(m_{1}\right)}^{\prime}$ and $\Psi$.

We want to show that there exists $n_{2}^{\prime}>l$ such that $\phi_{n_{1}, n_{2}^{\prime}}$ and $\phi_{l, n_{2}^{\prime}} \circ \alpha$ are approximately unitarily equivalent on $F_{1}$ to within $\epsilon_{1}$. Note that $\phi_{n_{1}, l}$ and $\alpha$ induce the same map on $V_{*}\left(E_{n_{1}}\right)$ and $\pi_{n_{1}}\left(F_{1}\right)$ is weakly approximately constant within $\varepsilon_{1} / 2$.

By conjugating a unitary, we may assume that $\phi_{n_{1}, l}$ and $\alpha$ agree on the central projections of $E_{n_{1}}$.

Let $H\left(=M_{k}\left(\mathcal{T}_{r}\right)\right)$ be a summand of $E_{n_{1}}$. Then $\phi_{n_{1}, l}\left(1_{H}\right)=\alpha\left(1_{H}\right)$, by the above assumption, where $1_{H}$ is the central projection corresponding to the summand $H$. We also have that $\left.\phi_{n_{1}, l}\right|_{H}$ and $\left.\alpha\right|_{H}$ induce the same map on $V_{*}(H)$. Suppose that $H^{\prime}$ is a summand of $E_{l}$ and $P^{\prime}$ is the corresponding central projection of $E_{l}$. Then $\left.P^{\prime} \phi_{n_{1}}\right|_{H}$ and $\left.P^{\prime} \alpha\right|_{H}$ induce the same map on $V_{*}(H)$.

Let $\gamma_{1}=\left.P^{\prime} \phi_{n_{1}}\right|_{H}$ and $\gamma_{2}=\left.P^{\prime} \alpha\right|_{H}$ be a pair of partial maps of $\phi_{n_{1}, l}$ and $\alpha$, respectively, from $H$ to a summand of $E_{l}$. Since $\gamma_{1}$ and $\gamma_{2}$ induce the same 
map on $V\left(I\left(E_{n_{1}}\right)\right), \gamma_{1}$ and $\gamma_{2}$ will be both injective or both non-injective. In the case of non-injective, they both factor through $Q(H)$ via $\gamma_{1 Q}$ and $\gamma_{2 Q}: Q(H) \rightarrow E_{l}$, respectively. Again, since $\gamma_{1}$ and $\gamma_{2}$ induce the same map on $V_{*}\left(E_{n_{1}}\right), \gamma_{1 Q}$ and $\gamma_{2 Q}$ both induce trivial $K_{1}$-map or both induce nontrivial $K_{1}$-map. (Notice that the $K_{1}$-maps are always trivial when the second summand comes from a non-trivial extension. In the case that the second summand does come from a trivial extension, $\pi_{l}$ induces an isomorphism on the $K_{1}$-groups.) Finally, it is clear that $\pi_{l} \circ \gamma_{1 Q}$ and $\pi_{l} \circ \gamma_{2 Q}$ are both zero or both are non-zero.

We consider four cases.

Case 1: $\gamma_{1}$ and $\gamma_{2}$ are injective.

Since $G_{1}$ contains the generators of $Q\left(E_{n_{1}}\right)$ and $\pi_{n_{1}}\left(F_{1}\right)$, it follows from (1) of the Theorem 4.10 that there exists a unitary $U_{\gamma} \in \gamma_{1}\left(1_{H}\right) E_{l} \gamma_{1}\left(1_{H}\right)$ such that $\gamma_{1}$ and $A d U_{\gamma} \circ \gamma_{2}$ are within $\epsilon_{1}$ on $F_{1} 1_{H}$. For any $n_{2}^{\prime}>l$, define $W_{\gamma}=\phi_{l, n_{2}}\left(U_{\gamma}\right)$. Then $\phi_{l, n_{2}^{\prime}} \circ \gamma_{1}$ and $A d W_{H} \circ \phi_{l, n_{2}^{\prime}} \circ \gamma_{2}$ are within $\epsilon_{1}$ on $F_{1} 1_{H}$.

Case 2: $\gamma_{1}$ and $\gamma_{2}$ are not injective and $\gamma_{1 Q}$ and $\gamma_{2 Q}$ induce non-trivial $K_{1}$ map.

First, $\gamma_{1 Q}$ and $\gamma_{2 Q}$ must be injective. (Here we note that if $\gamma$ is a noninjective homomorphism from $H$ to a $C^{*}$-algebra $A, \gamma$ factor through $\gamma_{Q}$ : $Q(H) \rightarrow A$. So $\gamma_{i Q}$ are two homomorphisms from $M_{k}\left(C\left(S^{1}\right)\right)$ to $E_{l}$, if $H=M_{k}\left(\mathcal{T}_{r}\right), i=1,2$.) Apply (2) of Theorem 4.10 and the above argument, we conclude that, for any $n_{2}^{\prime}>l$ there exists a unitary $W_{\gamma} \in$ $\phi_{l, n_{2}^{\prime}} \circ \gamma_{1}\left(1_{H}\right) E_{n_{2}^{\prime}} \phi_{l, n_{2}^{\prime}} \circ \gamma_{1}\left(1_{H}\right)$ such that $\phi_{l, n_{2}^{\prime}} \circ \gamma_{1}$ and $\operatorname{Ad} W_{\gamma} \circ \phi_{l, n_{2}^{\prime}} \circ \gamma_{2}$ are within $\epsilon_{1}$ on $F_{1} 1_{H}$.

Case 3: $\gamma_{1}$ and $\gamma_{2}$ are not injective, and $\gamma_{1 Q}$ and $\gamma_{2 Q}$ induce trivial $K_{1}$-map.

We claim that there are an integer $n_{2}(\gamma)>l$ and two homomorphisms $\psi_{1}$ and $\psi_{2}$ from $Q\left(E_{n_{1}}\right)$ into $Q\left(E_{n_{2}(\gamma)}\right)$ with finite dimensional ranges such that

$\left\|\psi_{1}(f)-\left[\phi_{l, n_{2}(\gamma)} \circ \gamma_{1}\right]_{Q}(f)\right\|<\varepsilon_{1} / 4$ and $\left\|\psi_{2}(f)-\left[\phi_{l, n_{2}(\gamma)} \circ \gamma_{2}\right]_{Q}(f)\right\|<\varepsilon_{1} / 4$

for every $f \in \pi_{n_{1}}\left(F_{1}\right) 1_{H}$. We note that since $\gamma_{i}$ is not injective, $\phi_{l, n_{2}(\gamma)} \circ \gamma_{i}$ factors through $\left[\phi_{l, n_{2}(\gamma)} \circ \gamma_{i}\right]_{Q}: Q(H) \rightarrow E_{l}$.

Now we prove the claim. We let $\left\{e_{i j}\right\}$ and $z$ be the generators of $Q(H)$. Note that the $C^{*}$-algebra $\left(\phi_{l, \infty}\right)_{Q}\left(e_{11}\right) Q(E)\left(\phi_{1, \infty}\right)_{Q}\left(e_{11}\right)$ has real rank zero ([BP] $]$. Since $\gamma_{i Q}$ induces trivial map on $K_{1}(H)$, for any $\eta>0$, by [Ln1], $\left[\phi_{l, \infty} \circ \gamma_{i}\right]_{Q}(z)$ is approximated by a unitary with finite spectrum, say $w_{i} \in$ $\left(\phi_{l, \infty}\right)_{Q}\left(e_{11}\right) E\left(\phi_{l, \infty}\right)_{Q}\left(e_{11}\right)$, within $\eta / 2$. Suppose that

$$
w_{i}=\sum_{j=1}^{m_{i}} \lambda_{j}^{(i)} p_{j}^{(i)}
$$


where $\left\{\lambda_{j}^{(i)}\right\}_{j=1}^{m_{i}} \in S^{1}$ and $\left\{p_{j}^{(i)}\right\}$ are mutually orthogonal projections (with sum $\left.\left(\phi_{l \infty}\right)_{Q}\left(e_{11}\right)\right)$ in $\left(\phi_{l \infty}\right)_{Q}\left(e_{11}\right) E\left(\phi_{l, \infty}\right)_{Q}\left(e_{11}\right), i=1,2$. By A8 in [Eff], we may assume, with additional error of $\eta / 2$,

$$
w_{i} \in\left[\phi_{l, n_{2}(\gamma)}\right]_{Q}\left(e_{11}\right) E_{n_{2}(\gamma)}\left[\phi_{l, n_{2}(\gamma)}\right]_{Q}\left(e_{11}\right)
$$

for some $n_{2}(\gamma)>l$.

Define the homomorphism $\psi_{i}: Q(H) \rightarrow E_{n_{2}(H)}$ by sending $e_{k j}$ to $\phi_{n_{1}, n_{2}(\gamma)}\left(e_{k j}\right)$ and $z$ to $w_{i}, i=1,2$. By choosing a sufficient small $\eta$, we can have inequalities in the claim.

Let $\left\{P_{j}\right\}$ be the (finite) set of central projections of $E_{n_{2}(\gamma)}$. By applying (1) of Theorem 4.11 to each $P_{j} \psi_{i}$, we obtain a unitary $U_{\gamma} \in \psi_{1}\left(1_{H}\right) E_{n_{2}(\gamma)} \psi_{1}\left(1_{H}\right)$ such that $\psi_{1}$ and $\operatorname{Ad} U_{\gamma} \circ \psi_{2}$ are within $\epsilon_{1} / 2$ on $\pi_{n_{1}}\left(F_{1} 1_{H}\right)$. For $f \in F_{1} 1_{H}$,

$$
\begin{aligned}
& \left\|\gamma_{1}(f)-\operatorname{Ad} U_{\gamma} \circ \gamma_{2}(f)\right\| \\
& =\left\|\gamma_{1}(f)-\psi_{1}(f)+\psi_{1}(f)-\operatorname{Ad} U_{\gamma} \gamma_{2}(f)-\operatorname{Ad} U_{\gamma} \circ \psi_{2}(f)+\operatorname{Ad} U_{\gamma} \circ \psi_{2}(f)\right\| \\
& <\epsilon / 4+\epsilon_{1} / 2+\epsilon_{1} / 4=\epsilon_{1} .
\end{aligned}
$$

Thus, for any $n_{2}^{\prime} \geq n_{2}(\gamma)>l$, let $W_{\gamma}=\phi_{n_{2}(\gamma), n_{2}^{\prime}}\left(U_{\gamma}\right)$, we have $\phi_{l, n_{2}^{\prime}} \circ \gamma_{1}$ and Ad $W_{\gamma} \circ \phi_{l, n_{2}^{\prime}} \circ \gamma_{2}$ are within $\varepsilon_{1}$ on $F_{1} 1_{H}$.

Case 4: $\pi_{l} \circ \gamma_{1 Q}$ and $\pi_{l} \circ \gamma_{2 Q}$ are trivial.

One applies (2) of Theorem 4.11. There exists a unitary $U_{H} \in \gamma_{1 Q}\left(1_{H}\right) E_{l} \gamma_{1 Q}\left(1_{H}\right)$ so that $\gamma_{1}$ and $\operatorname{Ad} U_{H} \circ \gamma_{2}$ are within $\epsilon_{1}$ on $F_{1} 1_{H}$. For any $n_{2}^{\prime}>l$, let $W_{\gamma}=\phi_{l, n_{2}^{\prime}}\left(U_{\gamma}\right)$. Then $\phi_{l, n_{2}^{\prime}} \circ \gamma_{1}$ and Ad $W_{H} \circ \phi_{l, n_{2}^{\prime}} \circ \gamma_{2}$ are within $\varepsilon_{1}$ on $F_{1} 1_{H}$.

Now let $n_{2}^{\prime}=\max \left\{n_{2}(\gamma), l+1\right\}$, where maximum is taking over all summands $H$ of $E_{n_{1}}$ and central projections $P^{\prime}$ of $E_{l}$. Define

$$
W=\sum W_{\gamma},
$$

where the sum is taking over all of summands $H$ of $E_{n_{1}}$ and the central projections $P^{\prime}$ of $E_{l}$. $W$ is a unitary in $E_{n_{2}^{\prime}}$. From above, a direct and simple computation shows that $\phi_{n_{1}, n_{2}^{\prime}}$ and $\operatorname{Ad} W \circ \phi_{l, n_{2}^{\prime}} \circ \alpha$ are within $\epsilon_{1}$ on $F_{1}$. We denote $\operatorname{Ad} W \circ \phi_{l, n_{2}^{\prime}} \circ \alpha$ by $\sigma_{1}$.

Let $F_{2} \subset E_{n_{2}^{\prime}}$ be a finite subset consisting of the images of the first $m_{1}$ elements of $\Delta_{(i)}, \Delta_{(j)^{\prime}}, i=1,2, \cdots, n_{2}^{\prime}$ and $j=1,2, \cdots, m_{1}^{\prime}$ along all possible routes. We also require that $\pi_{n_{2}^{\prime}}\left(F_{2}\right)$ contains the images of the generators of $Q\left(E_{m_{1}}^{\prime}\right)$. By Theorem 5.2, there exists $n_{2}>n_{2}^{\prime}$ such that $\pi_{n_{2}}\left(\phi_{n_{2}^{\prime}, n_{2}}\left(F_{2}\right)\right)$ is of weakly approximately constant to within $\epsilon_{2} / 2$. We will denote $\phi_{n_{2}^{\prime}, n_{2}}\left(F_{2}\right) \subset$ $E_{n_{2}}$ by $F_{2}$ again. We remark that $F_{2}$ contains the images of $F_{1}$ and $F_{1}^{\prime}$ along all possible routes. 
In summary, we have the following diagram:

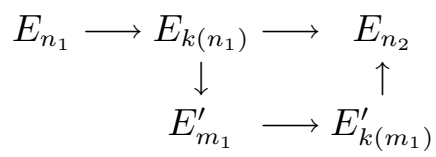

(1) approximately commutes on $F_{1}$ to within $\epsilon_{1}$,

(2) $\pi_{m_{1}}^{\prime}\left(F_{1}^{\prime}\right) \subset Q\left(E_{m_{1}}^{\prime}\right)$ is of weakly approximately constant to within $\epsilon_{1}^{\prime} / 2$,

(3) $Q\left(\psi_{m_{1}, k\left(m_{1}\right)}\right)\left(G_{1}^{\prime}\right)$ is of weakly approximately constant to within $\delta_{1}^{\prime} / 160$, and

(4) $\pi_{n_{2}}\left(F_{2}\right) \subset Q\left(E_{n_{2}}\right)$ is of weakly approximately constant to within $\epsilon_{2} / 2$.

Notice that the above diagram can be written as:

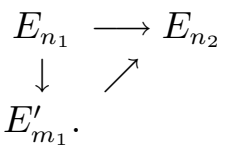

Next, we are going to repeat the above process. We want to find $m_{2}>m_{1}$ and a map from $E_{n_{2}}$ to $E_{m_{2}}^{\prime}$ so that the diagram:

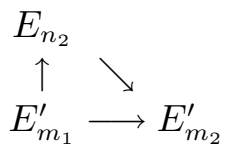

commutes on $F_{1}^{\prime}$ to within $\epsilon_{1}^{\prime}$. The map will take the form:

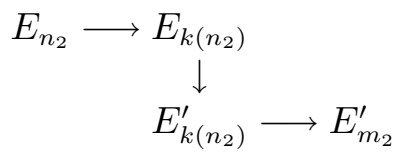

for some $k\left(n_{2}\right)>n_{2}$. Let $\Gamma_{2} \subset E_{n_{2}}$ be a finite subset such that $\Gamma_{2}$ contains $F_{2}$ and $\pi_{n_{2}}\left(\Gamma_{2}\right)$ contains the generators of $Q\left(E_{n_{2}}\right)$. Denote $\pi_{n_{2}}\left(\Gamma_{2}\right)$ by $G_{2}$. For fixed $E_{n_{2}}, \Gamma_{2}$ and $\epsilon_{2} / 2$, there exists $\delta_{2}$ as in Theorem 4.10. Let $k\left(n_{2}\right)>$ $n_{2}$ so that $Q\left(\phi_{n_{2}, k\left(n_{2}\right)}\right)\left(G_{2}\right)$ is of weakly approximately constant to within $\delta_{2} / 160$. Recall that $\alpha_{k\left(n_{2}\right)}$ is a $*$-homomorphism from $E_{k\left(n_{2}\right)}$ to $E_{k\left(n_{2}\right)}^{\prime}$. Now $Q\left(\psi_{m_{1}, k\left(n_{2}\right)}\right)$ and $Q\left(\alpha_{k\left(n_{2}\right)} \circ \phi_{n_{2}, k\left(n_{2}\right)} \circ \eta_{1}^{\prime} \circ \psi_{m_{1}, k\left(m_{1}\right)}\right)$ induce the same Ktheory map. So, by 5.2 , there exists $l>k\left(n_{2}\right)$ such that, up to an inner automorphism in $Q\left(E_{l}^{\prime}\right)$, the diagram:

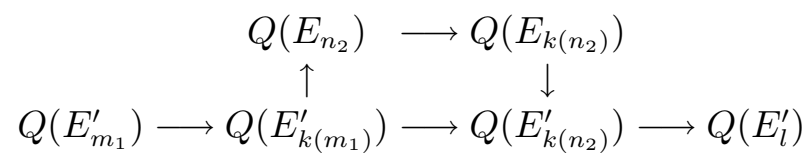


commutes on $G_{1}^{\prime}$ to within $\delta_{1}^{\prime} / 2$. This is because that the image of $G_{1}^{\prime} \subset$ $Q\left(E_{k\left(m_{1}\right)}^{\prime}\right)$ is already of approximately constant to within $\delta_{1}^{\prime} / 160$.

Denote by $\alpha_{k\left(n_{2}\right)}^{\prime}$ the composition of a suitable inner automorphism $\operatorname{Ad}\left(u^{\prime}\right)$ on $Q\left(E_{l}^{\prime}\right)$ with the composition of the map from $Q\left(E_{k\left(n_{2}\right)}\right)$ to $Q\left(E_{k\left(n_{2}\right)}\right)$ with the map from $Q\left(E_{k\left(n_{2}\right)}\right)$ to $Q\left(E_{l}\right)$ in the above diagram. We choose the inner automorphism so that the following diagram approximately commutative on $G_{1}$ within $\delta_{1}^{\prime} / 2$ :

$$
\begin{aligned}
& Q\left(E_{n_{2}}\right) \longrightarrow Q\left(E_{k\left(n_{2}\right)}\right) \\
& \uparrow \\
& \downarrow \alpha_{k\left(n_{2}\right)}^{\prime} \\
& Q\left(E_{m_{1}}^{\prime}\right) \longrightarrow Q\left(E_{k\left(m_{1}\right)}^{\prime}\right) Q\left(E_{k\left(n_{2}\right)}^{\prime}\right) \longrightarrow Q\left(E_{l}^{\prime}\right) .
\end{aligned}
$$

If $\Phi^{\prime}$ is the map from $E_{k\left(n_{2}\right)}$ to $E_{l}^{\prime}$ obtained from the above diagram, then

$$
\operatorname{Ad}\left(u^{\prime}\right) \circ Q\left(\Phi^{\prime}\right)=\alpha_{k\left(n_{2}\right)}^{\prime} .
$$

By the existence Theorem (3.1), there is a map $\Psi^{\prime}: E_{k\left(n_{2}\right)} \rightarrow E_{l}^{\prime}$ such that $Q\left(\Psi^{\prime}\right)=\alpha_{k\left(n_{2}\right)}^{\prime}$ and $\Psi^{\prime}$ and $\Phi^{\prime}$ induces the same map on $V_{*}\left(E_{k\left(n_{2}\right)}^{\prime}\right)$.

By repeating the above argument, applying 4.10 and 4.11 , we obtain an integer $m_{2}^{\prime}>l$ and a homomorphism from $E_{k\left(n_{2}\right)}$ into $E_{m_{2}^{\prime}}^{\prime}$ such that the following diagram is approximately commutative to within $\epsilon^{\prime}$ on $F_{1}^{\prime}$ :

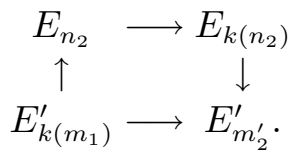

Let $F_{2}^{\prime} \subset E_{m_{2}^{\prime}}$ consist of the images of $F_{1}, F_{1}^{\prime}, F_{2}$ and the images of the first $n_{2}$ elements of $\Delta_{(1)}, \ldots, \Delta_{\left(n_{2}\right)}$ and $\Delta_{(1)^{\prime}}, \ldots, \Delta_{\left(m_{1}\right)^{\prime}}$ from all possible routes. We also require that $\pi_{m_{2}^{\prime}}(F)$ contains the generators of $Q\left(E_{m_{2}^{\prime}}\right)$. Let $m_{2}>m_{2}^{\prime}$ such that $\pi_{m_{2}}^{\prime} \circ \psi_{m_{2}^{\prime}, m_{2}}\left(F^{\prime}\right)$ is of weakly approximately constant to within $\epsilon_{2}^{\prime} / 2$. We will denote $\psi_{m_{2}^{\prime}, m_{2}}\left(F^{\prime}\right)$ by $F_{2}^{\prime}$ again. Hence we have a diagram

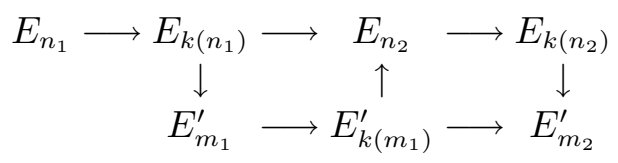

such that

(1) the first square commutes on $F_{1}$ to within $\epsilon_{1}$,

(2) the second square commutes on $F_{1}^{\prime}$ to within $\epsilon_{1}^{\prime}$,

(3) $Q\left(\phi_{n_{2}, k\left(n_{2}\right)}\right)\left(G_{2}\right)$ is of weakly approximately constant to within $\delta_{2} / 160$,

(4) $\pi_{n_{2}}\left(F_{2}\right) \subset Q\left(E_{n_{2}}\right)$ is of wearly approximately constant to within $\epsilon_{2} / 2$,

(5) $\pi_{m_{2}}^{\prime}\left(F_{2}^{\prime}\right) \subset Q\left(E_{m_{2}}^{\prime}\right)$ is of weakly approximately constant to within $\epsilon_{2}^{\prime} / 2$. 
Notice that if we combine these together and compress some of the algebras, we have

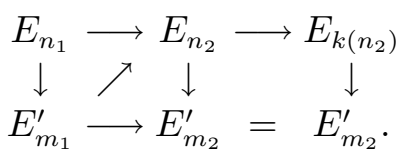

The first triangle is commutative on $F_{1}$ to within $\epsilon_{1}$ and the second is commutative on $F_{1}^{\prime}$ to within $\epsilon_{1}^{\prime}$.

By repeating the above process, we get two subsequences $\left\{E_{n_{k}}\right\}$ and $\left\{E_{m_{k}}^{\prime}\right\}$, two subsequences of finite sets $\left\{F_{k} \subset E_{n_{k}}\right\}$ and $\left\{F_{k}^{\prime} \subset E_{m_{k}}^{\prime}\right\}$ and the following not necessarily commutative diagram:

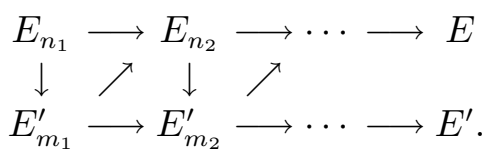

Here the up triangle in the $k$ th square is commutative on $F_{k}$ to within $\epsilon_{k}$ and the lower triangle is commutative on $F_{k}^{\prime}$ to within $\epsilon_{k}^{\prime}$. The images of the sets $\left\{F_{k}\right\}$ and $\left\{F_{k}^{\prime}\right\}$ in $E$ and $E^{\prime}$ are dense since they contain the images of all $\Delta_{(k)}$ and $\Delta_{(i)}^{\prime}$. This approximately intertwining satisfies the conditions of Theorem 2.2 [Ell2]. Hence it induces a $*$-isomorphism from $E$ to $E^{\prime}$. Clearly, $\phi$ induces $\eta$.

5.5. For $\mathbb{T}_{1}$-algebra, there is no trivial extension appears in the inductive limit systems $E=\lim _{\rightarrow}\left(E_{n}, \phi_{n, n+1}\right)$. By Corollary 2.11, a homomorphism from $V(E)$ to $V\left(E^{\prime}\right)$ extends uniquely to a homomorphism from $V_{*}(E)$ to $V_{*}\left(E^{\prime}\right)$. As a consequence of Lemma 5.4, we have the following:

Theorem. Let $E=\lim \left(E_{n}, \phi_{n, n+1}\right)$ and $E^{\prime}=\lim \left(E_{n}^{\prime}, \psi_{n, n+1}\right)$ be two $\mathbb{T}_{1}$ algebras. Suppose that $\eta: V(E) \rightarrow V\left(E^{\prime}\right)$ is an isomorphism such that $\eta\left(\left[1_{E}\right]\right)=\left[1_{E^{\prime}}\right]$. Then there exists a $*$-isomorphism $\phi: E \rightarrow E^{\prime}$ such that $\phi$ induces $\eta$.

5.6. Next, we will show that any $A \mathcal{T}$-algebra can be written as an inductive limit of finite direct sums of matrix algebras over $\mathcal{T}$-algebras with the additional condition on the connecting maps. Namely, it satisfies the requirement imposed in Lemma 5.4.

Lemma. For any subsequence $\left\{n_{k}\right\}$ of $\mathbf{N}$, there exists an increasing sequence $B_{k} \subset \mathcal{T}_{0}$ such that $\cup_{k}^{\infty} B_{k}$ is dense in $\mathcal{T}_{0}$, where $B_{k} \cong M_{n_{k}} \oplus C^{*}\left(U_{k}\right)$, and where $U_{k} \in \mathcal{T}_{0}$ is a normal partial isometry with its essential spectrum of the whole circle $S^{1}$.

Proof. (Note that $\cup_{k=1}^{\infty} M_{n}\left(B_{k}\right)$ is dense in $M_{n}\left(\mathcal{T}_{0}\right)$.) 
Proof. Fix a separable (infinite dimensional) Hilbert space $H$. Let $\left\{p_{i}\right\}$ be rank one projections in $B(H)$ with $\sum p_{i}=1$. Then $\mathcal{T}_{0}$ is generated by $\mathcal{K}$, the compact operators, and a unitary

$$
U=\sum \lambda_{i} p_{i}
$$

where $\left\{\lambda_{i}\right\}_{i=k}^{\infty}$ is dense in $S^{1}$ for each $k$.

Let $\left\{e_{i j}\right\}$ be a matrix unit for $\mathcal{K}$. Let $M_{n_{k}}$ be the algebra generated by $\left\{e_{i j}\right\}_{i, j \leq n_{k}}$. Denote $U_{k}=\sum_{i>n_{k}} \lambda_{i} p_{i}$. Then $M_{n_{k}}$ is orthogonal to $C^{*}\left(U_{k}\right)$ and

$$
B_{k}=M_{n_{k}} \oplus C^{*}\left(U_{k}\right) \hookrightarrow \mathcal{T}_{0} .
$$

We now show that $B_{k} \subset B_{k+1}$. Write

$$
U_{k}=\sum_{n_{k}<i \leq n_{k+1}} \lambda_{i} p_{i}+U_{k+1}
$$

It is clear that $U_{k}$ is then an element of $B_{k+1} . M_{n_{k}} \subset M_{n_{k+1}}$. So $B_{k} \hookrightarrow B_{k+1}$.

To show that $\mathcal{T}_{0}=\lim _{\rightarrow} B_{k}$, we notice that $U \in B_{k}$ for all $k$ and $\left\{v_{i j}\right\} \subset$ $\cup B_{k}$.

Proposition 5.7. Let $E=\lim \left(E_{n}, \phi_{n}\right)$ be an inductive limit $C^{*}$-algebra where each $E_{n}$ is a finite direct sum of matrix algebras over $\mathcal{T}$-algebras. Then $E$ is isomorphic to $E^{\prime}=\lim _{\rightarrow} E_{n}^{\prime}$ where each $E_{n}^{\prime}$ is a finite direct sum of matrix algebras over $\mathcal{T}$-algebras and where each connecting map from $E_{n}^{\prime}$ to $E_{n+1}^{\prime}$ satisfies the following: If $M_{k}\left(\mathcal{T}_{0}\right)$ is a summand of $E_{n}^{\prime}$, then the connecting map restricted to this block vanishes on $M_{k}(\mathcal{K})$.

Proof. We construct $E^{\prime}=\lim _{\rightarrow} E_{n}^{\prime}$ in several steps.

Step 1. We first replace those blocks that are isomorphic to matrix algebras over $\mathcal{T}_{0}$ by direct sums of matrix algebras and matrix algebras over $C\left(S^{1}\right)$.

Take $n_{k}=k$ in Lemma 5.6. Let $B_{k}=M_{k} \oplus C^{*}\left(U_{k}\right)$. Then $\mathcal{T}_{0}=\lim _{\rightarrow} B_{k}$.

For each $E_{n}$ we can write $E_{n}=E_{n}^{(1)} \oplus E_{n}^{(2)}$ where $E_{n}^{(2)}=L_{n} \otimes \overrightarrow{\mathcal{T}_{0}}, L_{n}$ is a finite dimensional $C^{*}$-algebra and there is no summand in $E_{n}^{(1)}$ which is a matrix algebra over $\mathcal{T}_{0}$. Clearly,

$$
E_{n}=E_{n}^{(1)} \oplus \lim _{\rightarrow} L_{n} \otimes B_{k} .
$$

Let $f^{(n)}=\left\{f_{k}^{(n)}\right\}$ be dense in $E_{n}$ and let $\epsilon_{n}=1 / 2^{n}$. Let $G_{1}=\left\{f_{1}^{(1)}\right\} \subset E_{1}$. There exists $n_{1}$ such that $\operatorname{dist}\left(f_{1}^{(1)}, E^{(1)} \oplus L_{1} \otimes B_{n_{1}}\right)<\varepsilon_{1}$. Fix $E_{1}^{0}=E_{1}^{(1)} \oplus B_{n_{1}}$. Let $h^{(1)}=\left\{h_{i}^{(1)}\right\}$ be dense in $E_{1}^{0}$. Let $H_{1}$ contain $h_{1}^{(1)}$ and the generators of $E_{1}^{0} . H_{1}$ is finite. 
Denote by $\phi_{1}$ the connecting map form $E_{1}$ to $E_{2}$. Let $p$ be the projection in $E_{2}$ corresponding to the summand $E_{2}^{(2)}$. By 2.4 (f), $\phi_{1} \cdot p$ vanishes on $I\left(E_{1}^{(1)}\right)$. Thus $\left.\phi_{1} \cdot p\right|_{E_{1}^{(1)} \oplus L_{1} \otimes B_{n_{1}}}$ factors through $Q\left(E_{1}^{(1)}\right) \oplus L_{1} \otimes B_{n_{1}}$, via, say $\bar{\psi}_{1}^{\prime}$. Denote by $q_{1}$ the quotient map from $E_{1}^{0}$ to $Q\left(E_{1}^{(1)}\right) \oplus L_{1} \otimes B_{n_{1}}$. By Lemma 4.2 [El12], there exists $n_{2}>n_{1}$ and there exists a unital $*$-homomorphism $\psi_{1}^{\prime}$ from $Q\left(E_{1}^{(1)}\right) \oplus L_{1} \otimes B_{n_{1}}$ to $E_{2}^{0}=E_{2}^{(1)} \oplus L_{2} \otimes B_{n_{2}}$ such that $\bar{\psi}_{1}^{\prime}$ and $\psi_{1}^{\prime}$ are within $\epsilon_{1}$ on $q_{1}\left(H_{1}\right)$. Let $\psi_{1}=\psi_{1} \cdot(1-p) \oplus \psi_{1}^{\prime} \circ q_{1}$, then

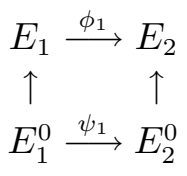

commutes on $H_{1}$ to within $\epsilon_{1}$. We may take $n_{2}$ large so that $E_{2}^{(1)} \oplus L_{2} \otimes B_{n_{2}}$ contains $G_{2}=\left\{\phi_{1}\left(G_{1}\right), \phi_{1}\left(f_{2}^{(1)}\right), f_{1}^{(2)}, f_{2}^{(2)}\right\}$ to within $\epsilon_{2}$.

Fix $E_{2}^{0}=E_{2}^{(1)} \oplus B_{n_{2}}$. Let $h^{(2)}=\left\{h_{i}^{(2)}\right\}$ be dense in $E_{2}^{0}$. Let $H_{2}$ contains

$$
\left\{\psi_{1}\left(H_{1}\right), \psi_{1}\left(h_{2}^{(2)}\right), h_{1}^{(2)}, h_{2}^{(2)}\right\}
$$

together with the generators of $q_{2}\left(E_{2}^{(1)}\right) \oplus B_{n_{2}}$ where $q_{2}$ is the quotient map from $E_{2}^{(1)}$ to $Q\left(E_{2}^{(1)}\right)$. As above, there exists $n_{3}>n_{2}$ and there exists a unital $*$-homomorphism $\psi_{2}$ form $E_{2}^{0}$ to $E_{3}^{0}=E_{3}^{(1)} \oplus B_{n_{3}}$ such that

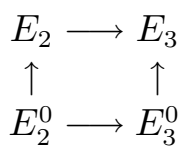

commutes on $H_{2}$ to within $\epsilon_{2}$ and $\operatorname{dist}\left(g, E_{3}^{0}\right)<\varepsilon_{3}$ for every $g \in G_{3}$, where $G_{3}$ contains $\phi_{2} \circ \phi_{1}\left(G_{1}\right), \phi_{2}\left(G_{2}\right), \phi_{2} \circ \phi_{1}\left\{f_{2}^{(1)}, f_{3}^{(1)}\right\}, \phi_{2}\left\{f_{2}^{(2)}, f_{3}^{(2)}\right\}, f_{1}^{(3)}, f_{2}^{(3)}, f_{3}^{(3)}$ to within $\epsilon_{3}$.

Continuing this way, we have

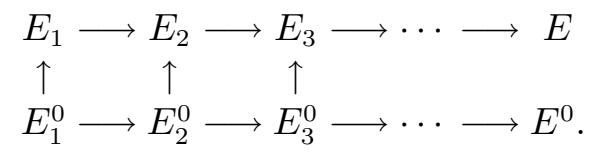

The above is an one-sided approximately intertwining in the sense of 2.3 [E112]. Hence it induces a unital $*$-homomorphism $\psi$ from $E^{0}$ to $E$. Furthermore, $\psi$ is surjective. This follows from the fact that the image of $E^{0}$ contains a dense set of $E$. To see that $\psi$ is injective, we note that each vertical map in the above diagram is injective. Hence, the induced map is injective.

Step 2. Replace these finite dimensional summands in each $E_{n}^{0}$ by matrix algebras over circle algebras. 
Write $E_{n}^{0}=D_{n} \oplus L_{n}$ where $L_{n}$ is finite dimensional and where $D_{n}$ is a finite direct sum of matrix algebras over $\mathcal{T}_{k}(k>0)$ or $C\left(S^{1}\right)$. Let $j_{n}$ : $L_{n} \hookrightarrow L_{n} \otimes C\left(S^{1}\right)$ be the canonical embedding and let $d_{n}: L_{n} \otimes C\left(S^{1}\right) \rightarrow L_{n}$ be evaluation at 1 . Notice $d_{n} \circ j_{n}=1$. Denote by $\psi_{n}: E_{n}^{0} \rightarrow E_{n+1}^{0}$. Then $\beta_{n}=j_{n+1} \circ \psi_{n} \circ d_{n}$ is a unital $*$-homomorphism from $D_{n} \oplus L_{n} \otimes C\left(S^{1}\right)$ to $D_{n+1} \oplus L_{n+1} \otimes C\left(S^{1}\right)$. Trivially, we have

$$
\begin{aligned}
& D_{n} \oplus L_{n} \quad \stackrel{\psi_{n}}{\longrightarrow} \quad D_{n+1} \oplus L_{n+1} \quad \stackrel{\psi_{n+1}}{\longrightarrow} \quad D_{n+2} \oplus L_{n+2} \\
& \uparrow d_{n} \quad j_{n+1} \downarrow \quad d_{n+2} \uparrow \\
& D_{n} \oplus L_{n} \otimes C\left(S^{1}\right) \stackrel{\beta_{n}}{\longrightarrow} D_{n+1} \oplus L_{n+1} \otimes C\left(S^{1}\right) \stackrel{\beta_{n+1}}{\longrightarrow} D_{n+2} \oplus L_{n+2} \otimes C\left(S^{1}\right)
\end{aligned}
$$

commutes. Hence

$$
\lim _{\rightarrow}\left(D_{n} \oplus L_{n} \otimes C\left(S^{1}\right), \beta_{n}\right)=E .
$$

Now $E$ is an inductive limit of finite direct sums of matrix algebras over $\mathcal{T}_{k}(k>0)$ or $C\left(S^{1}\right)$. We write $D_{n} \oplus L_{n} \otimes C\left(S^{1}\right)=R_{n} \oplus K_{n}$ where $K_{n}=$ $L_{n}^{\prime} \otimes C\left(S^{1}\right)$ with $L_{n}^{\prime}$ finite dimensional and where $R_{n}$ is a finite direct sum of matrix algebra over $\mathcal{T}_{k}(k>0)$.

Step 3. To replace $K_{n}$ by $L_{n}^{\prime} \otimes \mathcal{T}_{0}$ with desired condition on the connecting maps.

Let $E_{n}^{\prime}=R_{n} \oplus L_{n}^{\prime} \otimes \mathcal{T}_{0}$. Then we have trivial extension

$$
0 \longrightarrow L_{n}^{\prime} \otimes \mathcal{K} \longrightarrow L_{n}^{\prime} \otimes \mathcal{T}_{0} \longrightarrow K_{n} \longrightarrow 0 .
$$

Let $\pi_{n}: L_{n}^{\prime} \otimes \mathcal{T}_{0} \longrightarrow K_{n}$ be the quotient map and let $i_{n}: K_{n} \longrightarrow L_{n}^{\prime} \otimes \mathcal{T}_{0}$ be the splitting such that $\pi_{n} \circ i_{n}=1$. Set $\pi_{n}^{\prime}=i d_{R_{n}} \oplus \pi_{n}$ and $i_{n}^{\prime}=i d_{R_{n}} \oplus i_{n}$. Then

$$
E=\lim _{\rightarrow}\left(R_{n} \oplus L_{n}^{\prime} \otimes \mathcal{T}_{0}, i_{n+1}^{\prime} \circ \beta_{n} \circ \pi_{n}^{\prime}\right) .
$$

Now $E_{n}^{\prime}=R_{n} \oplus L_{n}^{\prime} \otimes \mathcal{T}_{0}$ is a finite direct sum of matrix algebras over $\mathcal{T}$ algebras. Furthermore, $\alpha_{n}=i_{n+1}^{\prime} \circ \beta_{n} \circ \pi_{n}^{\prime}$ vanishes on $I\left(L_{n}^{\prime} \otimes \mathcal{T}_{0}\right)$.

Remark 5.8. We would like to point out that a $C^{*}$-algebra $E \in \mathbb{T}$ is an $A \mathbb{T}$-algebra if and only if the third variable in $V_{*}(E)$ is zero, i.e., $d([u])=0$ for every $[u] \in k(E)_{+}$. Write $0 \rightarrow I(E) \rightarrow E \rightarrow Q(E) \rightarrow 0$. Then the above statement follows immediately from Theorem 5 in $[\mathbf{L R}]$, since the map $\delta_{0}$ there will be zero, if $d=0$, and $\delta_{1}=0$ since $E$ has real rank zero. Therefore $E$ is an $A F$-algebra if and only $k(E)_{+}=\{0\}$, since $k(E)_{+}=\{0\}$ implies that $d=0$ and $K_{1}(E)=\{0\}$.

\section{References}

[Ar] W. Arveson, Notes on extensions of $C^{*}$-algebras, Duke J. Math., 44 (1977), 329-355. 
[BBEK] B. Blackadar, O. Bratteli, G.A. Elliott and A. Kumjian, Reduction of real rank in inductive limits of $C^{*}$-algebras, Math. Ann., 292 (1992), 240-247.

[BEEK] O. Bratteli, G.A. Elliott, D.E. Evans and A. Kishimoto, On classification of $C^{*}$ algebras of real rank zero, III: The infinite case, in preparation.

[Bn] L.G. Brown, The universal coefficient theorem for Ext and quasidiagonality, Proc. International Conf. on Operator Algebras and Group Representations, Neptun, Romania, 1980.

[BD] L.G. Brown and M. Dadarlat, Extensions of $C^{*}$-algebras and quasidiagonality, preprint.

[BDF1] L.G. Brown, R. Douglas and P. Fillmore, Unitary equivalence modulo the compact operators and extensions of $C^{*}$-algebras, Proc. Conf. on Operator Theory, Springer Lecture Notes in Math., 345 (1973), 56-128.

[BDF2] Extensions of $C^{*}$-algebras and K-homology, Ann. of Math., 105 (1977), 265-324.

[BP] L.G. Brown and G.K. Pedersen, $C^{*}$-algebras of real rank zero, J. Funct. Anal., 99 (1991), 131-149.

[C] J. Cuntz, K-theory for certain $C^{*}$-algebras, Ann. Math., 113 (1981), 181-197.

[D1] M. Dadarlat, Reduction to dimension three of local spectra of real rank zero $C^{*}$ algebras, J. Reine Angew. Math., 460 (1995), 189-212.

[D2] Approximately unitarily equivalent morphisms and inductive limit $C^{*}$-algebras, K-Theory, 9 (1995), 117-137.

[DL1] M. Dadarlat and T. Loring, Extensions of certain real rank zero $C^{*}$-algebras, preprint.

[DL2] Classifying $C^{*}$-algebras via ordered, mod-p K-theory, preprint.

[Eff] E. Effros, Dimensions and $C^{*}$-algebras CBMS regional conf. Ser. in Math., no. 46, Amer. Math. Soc., Providence, R.I., 1-74.

[Ell1] G.A. Elliott, On the classification of inductive limits of sequences of semisimple finite-dimensional algebras, J. Algebra, 38 (1876), 29-44.

[Ell2] - On the classification of $C^{*}$-algebras of real rank zero, J. Reine Angew. Math., 443 (1993), 179-219.

[Ell3] _ A classification of certain simple $C^{*}$-algebras, pp. 373-385 in: Quantum and Non-Commutative Analysis (edited by H. Araki et al.), Kluwer, Dordrecht, 1993.

[Ell4] _ A classification of certain simple $C^{*}$-algebras, II, Ann. of Math., to appear.

[El15] _ Are amenable $C^{*}$-algebras classifiable?, Representation Theory of Groups and Algebras, Contemporary Math., Vol. 145, Amer. Math. Soc., Providence RI., 1993.

[Ell6] _ Dimension groups with torsion, International J. Math., 4 (1990), 361-380.

[EE] G.A. Elliott and D.E. Evans, The structure of irrational rotation $C^{*}$-algebras, Ann. Math., 138 (9193), 477-501.

[EES] G.A. Elliott, D.E. Evans and H. Su, Classification of inductive limits of matrix algebras over the Toeplitz algebras, preprint.

[EG1] G.A. Elliott and G. Gong, On inductive limits of matrix algebras over two-tori, Amer. J. Math., 118 (1996), 263-290. 
[EG2] _ On the classification of $C^{*}$-algebras of real rank zero, II, Ann. of Math., to appear.

[EGL] G.A. Elliott, G. Gong and L. Li, On simple inductive limits of matrix algebras over higher dimensional spaces, II, in preparation.

[EGLP] G.A. Elliott, G. Gong, H. Lin and C. Pasnicu, Abelian $C^{*}$-subalgebras of $C^{*}$-algebras of real rank zero and inductive limit $C^{*}$-algebras, Duke Math. J., to appear.

[ER] G.A. Elliott and M. Rørdam, Classification of certain infinite simple $C^{*}$-algebras, II, Comment. Math. Helrctici, 70 (1995), 615-638.

[G1] G. Gong, Classification of $C^{*}$-algebras of real rank zero and unsuspended E-equivalent types, preprint.

[G2] - On inductive limits of matrix algebras over higher dimensional spaces, part I, Math. Scand., to appear.

[G3] On inductive limits of matrix algebras over higher dimensional spaces, part II, Math. Scand., to appear.

[KR] R.V. Kadison and J.R. Ringrose, Derivations and automorphisms of operator algebras, Comm. Math. Phys., 4 (1967), 32-63.

[K] Kirchberg, in preparation.

[Li] L. Li, Classification of simple $C^{*}$-algebras: Inductive limits of matrix algebras over 1-dimensional spaces, Ph.D. thesis, Univ. of Toronto.

[Ln1] H. Lin, Exponential rank and Brown-Pedersen's Conjectures, J. Funct. Anal., 114 (1993), 1-11.

[Ln2] - Approximation by normal elements with finite spectra in $C^{*}$-algebras of real rank zero, Pacific. J. Math., 173 (1996), 443-489.

[Ln3] - Almost commuting unitaries and classification of purely infinite simple $C^{*}$ algebras, preprint.

[Ln4] - Almost commuting unitaries in purely infinite simple $C^{*}$-algebras, Math. Ann., 303 (1995), 599-616.

[Ln5] Classification of $C^{*}$-algebras of real rank zero with zero $K_{1}$, J. Operator Theory, 35 (1996), 147-178.

[LP1] H. Lin and N.C. Phillips, Classification of direct limits of even Cuntz-circle algebras, Mem. Amer. Math. Soc., 118 (1995), no. 565.

[LP2] - Approximate unitarily equivalence of homomorphisms from $\mathcal{O}_{\infty}$, J. Reine Angew. Math., 464 (1995), 173-186.

[LP3] Almost multiplicative morphisms and Cuntz-algebra $\mathcal{O}_{2}$, J. International Math., 6 (1995) 625-643.

[LR] H. Lin and M. Rørdam, Extensions of inductive limits of circle algebras, J. London Math. Soc., 51 (1995), 603-613.

[Lr] T.A. Loring, $C^{*}$-algebras generated by stable relations, J. Funct. Anal., 112 (1993), 159-203.

[Pd] G.K. Pedersen, $C^{*}$-algebras and Their Automorphism Groups, Academic Press, London/New York/San Francisco, 1979.

[Ph1] N. C. Phillips, Approximate unitarily equivalence of homomorphisms from odd Cuntz-algebras, preprint. 
[Ph2] _ A classification theorem for nuclear purely infinite simple $C^{*}$-algebras, preliminary preprint.

[Rr1] M. Rørdam, Classification of inductive limits of Cuntz algebras, J. Reine Angew. Math., 440 (1993), 175-200.

[Rr2] Classification of Cuntz-Krieger algebras, K-Theory, to appear.

[Rr3] , A short proof of Elliott's Theorem: $\mathcal{O}_{2} \otimes \mathcal{O}_{2} \cong \mathcal{O}_{2}$, C. R. Math. Rep. Acad. Sci. Canada, XVI, 31-36.

[Rr4] , Classification of certain infinite simple $C^{*}$-algebras, J. Funct. Anal., preprint.

[Rr5] - Classification of certain infinite simple $C^{*}$-algebras, III, preprint.

[RS] J. Rosenberg and C. Schochet, The Künneth theorem and the universal coefficient theorem for Kasparov's generalized K-functor, Duke Math. J., 55 (1987), 431-474.

[Su1] H. Su, On the classification of $C^{*}$-algebras of real rank zero: Inductive limits of matrix algebras over non-Hausdorff graphs, Memoirs, Amer. Math. Soc., no. 547, Vol. 114.

[Su2] _ Classification for certain real rank zero $C^{*}$-algebras, J. Funct. Anal., to appear.

[Tm] K. Thomsen, On isomorphisms of inductive limits of $C^{*}$-algebras, Proc. Amer. Math. Soc., 113 (1991), 947-953.

[V] D. Voiculescu, A non-commutative Weyl-von Neumann Theorem, Rev. Roum. Math. Pures et Appl., 21 (1976), 97-113.

[Zh1] S. Zhang, $C^{*}$-algebras with real rank zero and the internal structure of their corona and multiplier algebras, Part III, Canad. J. Math., 62 (1990), 159-190.

[Zh2] _ K K-groups, quasidiagonality, and interpolation by multiplier projections, Trans. Amer. Math. Soc. 325 (1991), 793-818.

Received February 5, 1996 and revised August 23, 1996. This research was partially supported by NSF grants DMS 93-01082 for the first author and a NSERC postdoctoral fellowship of Canada for the second author.

UNIVERSITY OF OREGON

EUGENE, OR 97403-1222

E-mail address: hlin@darkwing.uoregon.edu

AND

The Fields Institute

Toronto, Canada M5T 3J1

Note: The Reference to Lemma 2.14 on page 127 appeared as "Corollary 2.14" IN THE PRINTED VERSION. 